\title{
Spin-Orbitronics at Transition Metal Interfaces
}

\author{
A. Manchon ${ }^{\mathrm{a}, \mathrm{b}, *}$, A. Belabbes ${ }^{\mathrm{a}}$ \\ ${ }^{a}$ Physical Science and Engineering Division (PSE), King Abdullah University of Science \\ and Technology (KAUST), Thuwal 23955, Saudi Arabia. \\ ${ }^{b}$ Computer, Electrical and Mathematical Science and Engineering Division (CEMSE), King \\ Abdullah University of Science and Technology (KAUST), Thuwal 23955, Saudi Arabia.
}

\begin{abstract}
The presence of large spin-orbit interaction at transition metal interfaces enables the emergence of a variety of fascinating phenomena that have been at the forefront of spintronics research in the past ten years. The objective of the present Chapter is to offer a review of these various effects from a theoretical perspective, with a particular focus on spin transport, chiral magnetism and their interplay. After a brief description of the orbital hybridization scheme at transition metal interfaces, we address the impact of spin-orbit coupling on the interfacial magnetic configuration, through the celebrated Dzyaloshinskii-Moriya interaction. We then discuss the physics of spin transport and subsequent torques occurring at these interfaces. We particularly address the spin Hall, spin swapping and inverse spin galvanic effects. Finally, the interplay between flowing charges and chiral magnetic textures as well as their induced dynamics are presented. We conclude this chapter by proposing some perspectives on promising research directions.
\end{abstract}

Keywords: Spin-Orbit Coupling, Spin Transfer Torque, Spin Hall Effect, Rashba effect, Dzyaloshinskii-Moriya interaction, Skyrmions

*aurelien.manchon@kaust.edu.sa 


\section{Contents}

1 Introduction 3

2 Transition Metal Interfaces 4

2.1 The transition metal series . . . . . . . . . . . . . . 4

2.2 Magnetic properties of transition metal interfaces . . . . . . . 6

2.3 Interfacial spin-orbit coupling . . . . . . . . . . . . . 8

3 Dzyaloshinskii-Moriya Interaction $\quad 13$

3.1 General principle . . . . . . . . . . . . . . . . . . . 13

3.2 Kashid's trimer model . . . . . . . . . . . . . . . . . . . . . . . 13

3.3 First principles methods . . . . . . . . . . . . . . . 18

3.4 DMI at $3 d / 5 d$ interfaces . . . . . . . . . . . . . . . . . . 19

3.5 Controlling DMI . . . . . . . . . . . . . . . . 26

4 Spin-Orbit Torques $\quad 29$

4.1 Overview . . . . . . . . . . . . . . . . . . 29

4.2 Spin Hall and swapping effects . . . . . . . . . . . . . 30

4.2.1 Impurity scattering driven spin Hall effect . . . . . . . . . 31

4.2.2 Intrinsic spin Hall effect in transition metals . . . . . . . . 34

4.2.3 Interfacial spin Hall effect . . . . . . . . . . . . . . . 35

4.2 .4 Spin swapping . . . . . . . . . . . . . . . . 35

4.2.5 Spin Hall and swapping torques . . . . . . . . . . . . . . 37

4.3 Inverse spin galvanic effect . . . . . . . . . . . . . . . . . 42

4.3.1 Inverse spin galvanic effect at realistic interfaces . . . . 44

5 Unconventional dynamics $\quad 47$

5.1 Chiral Damping . . . . . . . . . . . . . . . . . . 47

5.1.1 Fundamentals of magnetic damping . . . . . . . . . 47

5.1 .2 Phenomenological theory of chiral damping . . . . . . . 50

5.1 .3 Microscopic origin of chiral damping . . . . . . . . . . 51

5.2 Topological Torques . . . . . . . . . . . . . . . . . 53

5.2 .1 Emergent Electrodynamics . . . . . . . . . . . . 54

5.2 .2 Topological spin torque . . . . . . . . . . . . 55

5.2 .3 Tight-binding model . . . . . . . . . . . . . 57

6 Conclusion $\quad 59$ 


\section{Introduction}

Spin-orbit coupling lies at the core of condensed matter. It is central to magnetism and spintronics, where it drives magnetic anisotropy [1], spin relaxation [2], magnetic damping [3], anisotropic magnetoresistance [4] and anomalous Hall effect [5]. Quite surprisingly, in spite of its already long history, this fundamental interaction has been pivotal to several revolutions in the past ten years. As a matter of fact, all the effects mentioned above exist in systems where inversion symmetry is preserved. But when inversion symmetry is broken, such as in certain classes of magnetic crystals or at interfaces, spin-orbit coupling triggers a number of fascinating phenomena such as antisymmetric magnetic exchange giving rise to topologically non-trivial magnetic textures, spin-momentum locking, spin-orbit torques, chiral magnetic damping etc. This broad area of research is called spin-orbitronics $[6,7]$.

Because of the coexistence of strong spin-orbit coupling and magnetism, transition metals have occupied a prominent position in the development of spinorbitronics. The interplay between current-driven spin-orbit torques and chiral magnetic texture at transition metal interfaces has resulted in very fast currentdriven magnetization reversal $[8,9,10]$, ultrafast domain wall propagation [11, $12]$ and current-driven skyrmion motion $[13,14,15,16]$. Ensuring a good control of these properties requires a fine understanding of the orbital hybridization occurring at the interface, as well as an accurate description of the various mechanisms at stake.

In this Chapter, we discuss the theoretical aspects of spin transport and magnetism at transition metal interfaces. Section 2 reviews the magnetism of bulk transition metals and transition metal interfaces and discuss the nature of the interfacial orbital hybridization in these systems. Section 3 addresses the microscopic origin of Dzyaloshinskii-Moriya interaction. Section 4 discusses the physics of spin-orbit torques, with a particular emphasis on spin Hall effect and inverse spin galvanic effect. Section 5 presents recent advances on the description of chiral magnets dynamics, and finally conclusions are given in Section 6 . 

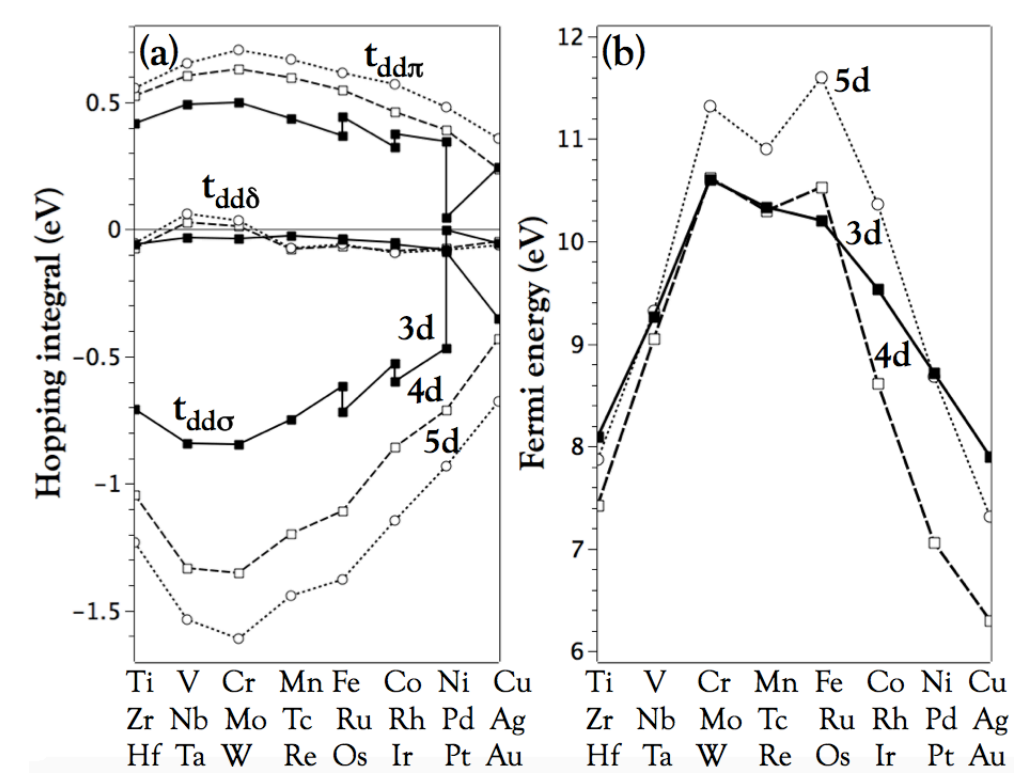

Figure 1: (a) Tight-binding parameters of transition metals and (b) Fermi energy, as parametrized in Ref. [17]. Notice that for Co, Fe and Ni, the hopping parameters duplicate due to spontaneous ferromagnetism.

\section{Transition Metal Interfaces}

\subsection{The transition metal series}

Transition metals occupy the central region of Mendeleev's table, extending along three series from Sc $3 d^{1} 4 s^{2}$ to $\mathrm{Cu} 3 d^{10} 4 s^{1}$ and $\mathrm{Zn} 3 d^{10} 4 s^{2}$ ( $3 d$ series), from Y $4 d^{1} 5 s^{2}$ to $\mathrm{Ag} 4 d^{10} 5 s^{1}$ and $\mathrm{Cd} 4 d^{10} 5 s^{2}$ (4d series), and from La $5 d^{1} 6 s^{2}$ to $\mathrm{Au} 5 d^{10} 6 s^{1}$ and $\mathrm{Hg} 4 d^{10} 5 s^{2}$ ( $5 d$ series). Because of the proximity in energy of the (1-n) $d$ and ns shells, they display multiple oxidation states that make their chemistry particularly interesting, leading to a wide variety of transition metal oxides and organometallic compounds. While $\mathrm{M}^{2+}$ and $\mathrm{M}^{3+}$ oxidation states are quite common in the $3 d$ series, higher oxidation states are more usual in the $4 d$ and $5 d$ series.

While magnetism in isolated transition metal ions is governed by Hund's rules, the magnetic properties of bulk transition metals is determined by band magnetism and Stoner criterion [18]. In a nutshell, Stoner criterion states that magnetism emerges as a compromise between kinetic energy and exchange interaction. The criterion reads $\operatorname{In}\left(\epsilon_{\mathrm{F}}\right)>1$, where $I$ is Stoner exchange parameter and $n\left(\epsilon_{\mathrm{F}}\right)$ is the density of states at Fermi energy. To understand how bulk magnetism develops in transition metals, it is instructive to inspect the behavior of the density of states of their $d$ orbitals. As a simple rule of thumb, on the one hand, throughout one given series, the $d$-orbital becomes more localized as the electron number increases $\left(\mathrm{n} d^{1} \rightarrow \mathrm{n} d^{10}\right)$ and correspondingly, the bandwidth 
decreases with the electron filling. On the other hand, upon increasing the series index $\left(3 d^{n} \rightarrow 5 d^{n}\right)$ the orbital gets more delocalized and as such more sensitive to the crystal field, resulting in $d$-orbital splitting and bandwidth broadening. This behavior is nicely represented by the evolution of the nearest neighbor hopping energies, as reported in Ref. [17] and collected in Fig. 1(a). It is clear that nearest neighbor hopping increases systematically from one series to another, $t_{5 d}>t_{4 d}>t_{3 d}$, but decreases when increasing the orbital filling within one given series. Of course this rule is simply qualitative, as the bandwidth also depends on the coordination number (it is larger for fcc than for bcc crystals for instance) and is influenced by spd hybridization as well [18]. Fig. 1(b) shows the behavior of Fermi level throughout the three series, where the maximum is obtained around half-filling of the $d$ bands.
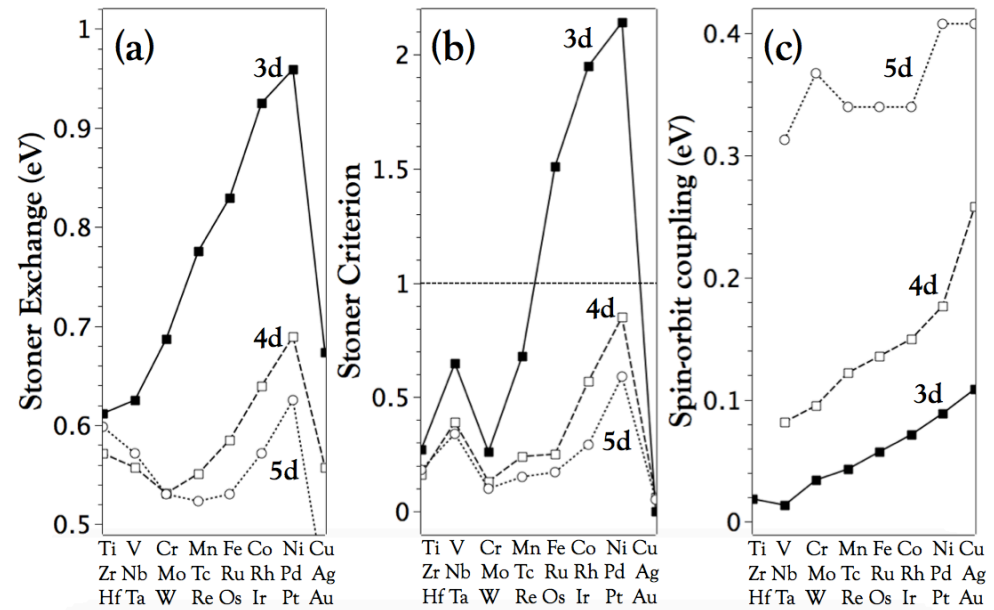

Figure 2: (a) Stoner exchange, (b) Stoner criterion and (c) spin-orbit coupling energy, as parametrized in Ref. [17]. As expected, $\mathrm{Co}, \mathrm{Fe}$ and $\mathrm{Ni}$ are the only three elements fulfilling Stoner criterion in the bulk.

The systematic increase of the hopping parameters when increasing the shell index has a direct consequence in terms of magnetism. Indeed, the exchange integral $I$ is an intra-atomic interaction, and it should scale with the localization of the orbital considered. Hence, one would expect $I_{3 d}>I_{4 d}>I_{5 d}$, as demonstrated in Fig. 2(a). Furthermore, since the density of states is roughly inversely proportional to the bandwidth, one expects $n_{3 d}\left(\epsilon_{\mathrm{F}}\right)>n_{4 d}\left(\epsilon_{\mathrm{F}}\right)>n_{5 d}\left(\epsilon_{\mathrm{F}}\right)$ and naturally, $3 d$ elements are more likely to be magnetic than any other $4 d$ and $5 d$ elements. The calculated Stoner criterion for transition metals is reported in Fig. 2(b) and one can see that the criterion is in fact only satisfied for Fe, Ni and Co. Other $3 d$ elements such as $\mathrm{Mn}, \mathrm{Cr}$ or $\mathrm{V}$ are not too far from fulfilling the Stoner criterion and indeed show enhanced susceptibility to magnetism. For instance, $\mathrm{Mn}$ and $\mathrm{Cr}$ (as well as $\gamma$-Fe) display antiferromagnetism. A phenomenological estimation can be obtained based on Bethe-Slater argument stating that antiferromagnetism occurs upon reducing the inter-atomic distance. This picture 
is supported by ab initio calculation on $3 d$ monolayers deposited on $4 d$ and $5 d$ substrates [19].

Interestingly, Rh, Pd, and Pt are fairly close to fulfilling the Stoner criterion so that a slight modification of their environment (say, reduction of coordination number due to the presence of an interface) can lead to the onset of magnetism, as discussed below. Finally, of most importance for our topic, the atomic spinorbit coupling also dramatically depends on the transition metal series, see Fig. 2 (c). Indeed, the spin-orbit coupling parameter increases with $Z$, such that $5 d$ transition metals possess a much larger spin-orbit coupling than their $4 d$ and $3 d$ counterparts. Combined with their high sensitivity to magnetism, this property makes $4 d$ and $5 d$ interfaces quite appealing for spin-orbitronics.

\subsection{Magnetic properties of transition metal interfaces}

At surfaces and interfaces, the coordination number reduces resulting in bandwidth narrowing for the out-of-plane orbitals [compare, for instance, the Co density of states reported on Fig. 4(a) and Fig. 4(c)]. As a result, the density of states at Fermi energy increases, which enhances Stoner criterion [18]. When two transition metal surfaces are put in contact, the interfacial orbital overlap also modifies further the $d$-orbital bandwidth leading to significant change in the magnetic properties of the interface (see below).
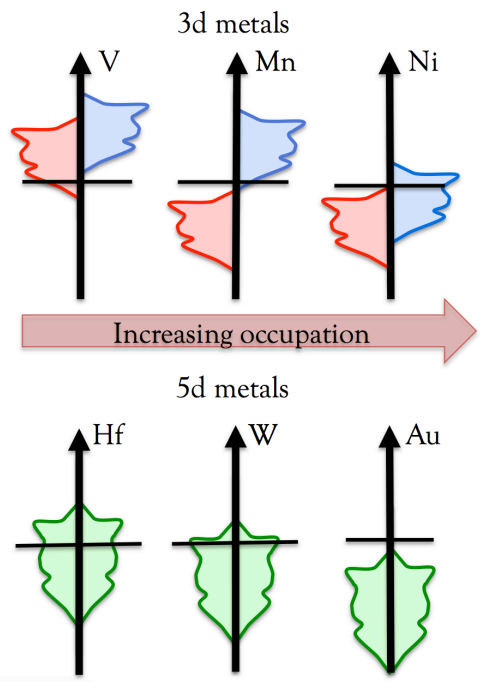

Figure 3: Schematic evolution of the $d$-orbital density of states upon increasing the orbital occupation for selected $3 d$ and $5 d$ monolayers. The energy position of the $3 d$ orbitals are mostly governed by Hund's rule, leading to a maximum spin-splitting for Mn elements and reduced spin-splitting at the two edges of the series, $\mathrm{V}$ and Ni. On the other hand, since $5 d$ orbitals are much broader and governed by crystal field splitting, they do not show any splitting and are simply pushed below the Fermi level upon increasing the orbital occupation.

To better understand the orbital hybridization taking place at transition metal interfaces, let us consider the evolution of the energy position of the 
density of states of selected $3 d$ and $5 d$ monolayers, as illustrated on Fig. 3 . Because of their large bandwidth, $4 d$ and $5 d$ elements do not show exchange splitting as expected and increasing the electronic filling pushes the density of states below the Fermi energy. As a results, $\mathrm{Ag}$ and $\mathrm{Au}$ display a $d$-orbital density of states located below Fermi energy. In contrast, $3 d$ monolayers show a slightly different behavior. The density of states is also pushed down through Fermi level when increasing the number of electrons, but at the same time its exchange splitting also depends on orbital filling. When increasing the number of electrons from $\mathrm{V}$ to $\mathrm{Mn}$, the exchange splitting increases while it decreases when going from Mn to Ni. This behavior can be understood following Hund's third rule that governs magnetism in isolated ions [20, 21]: Mn ion has a high spin configuration with 5 unpaired electrons, while $\mathrm{Ni}$ and $\mathrm{V}$ ions have a low spin configuration. The consequence is that $\mathrm{V}$ and $\mathrm{Ni}$, on both sides of the $3 d$ series show a weak spin splitting while a large amount on one spin species is present at the Fermi level. In contrast, Mn has a large spin splitting but Fermi energy lies in-between the two spin species, leading to a reduced amount of $3 d$ electrons at Fermi level.
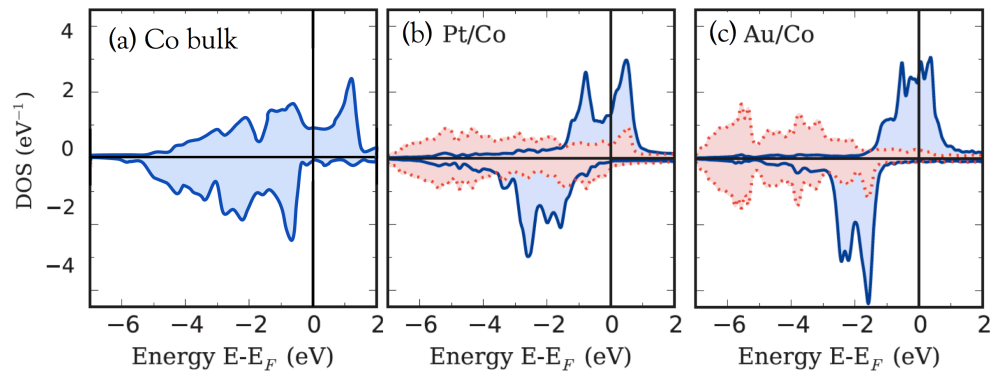

Figure 4: Density of states of (a) bulk fcc Co, (b) Co overlayer and topmost Pt layer in $\mathrm{Co} / \mathrm{Pt}(111)$ interface, and (c) Co overlayer and topmost $\mathrm{Au}$ layer in $\mathrm{Co} / \mathrm{Au}(111)$ interface. The interfaces consist in one monolayer of Co deposited on six layers of Pt or Au. From Ref. $[22]$.

Let us now consider the interface between $3 d$ and $4(5) d$ layers. Because of the different in energy position of the $3 d$ and $4(5) d$ states, different material combinations give rise to different hybridization schemes, as illustrated in Fig. 4. For instance, since the $d$-orbitals of $\mathrm{Cu}, \mathrm{Au}$ and $\mathrm{Ag}$ are located far below Fermi level, they do not (or only very weakly) hybridize with the $d$-orbitals of the $3 d$ elements deposited on top [Fig. 4(c)]. In contrast, other elements such as $\mathrm{Pt}$ or $\mathrm{W}$ possess a large density of states and are likely to hybridize strongly with the $3 d$ overlayer [Fig. 4(b)]. Nonetheless, such hybridization depends on the $3 d$ metal: it is stronger for elements at the edges of the $3 d$ series $(\mathrm{V}, \mathrm{Ni})$, than for the ones in the center $(\mathrm{Mn})$. The latter have much less $3 d$ states available at Fermi energy due to the large exchange splitting, see Fig. 3.

An interesting consequence is that upon such an orbital hybridization, (i) the $4(5) d$ substrate becomes magnetized by proximity effect $[23,24,25,26]$, while 

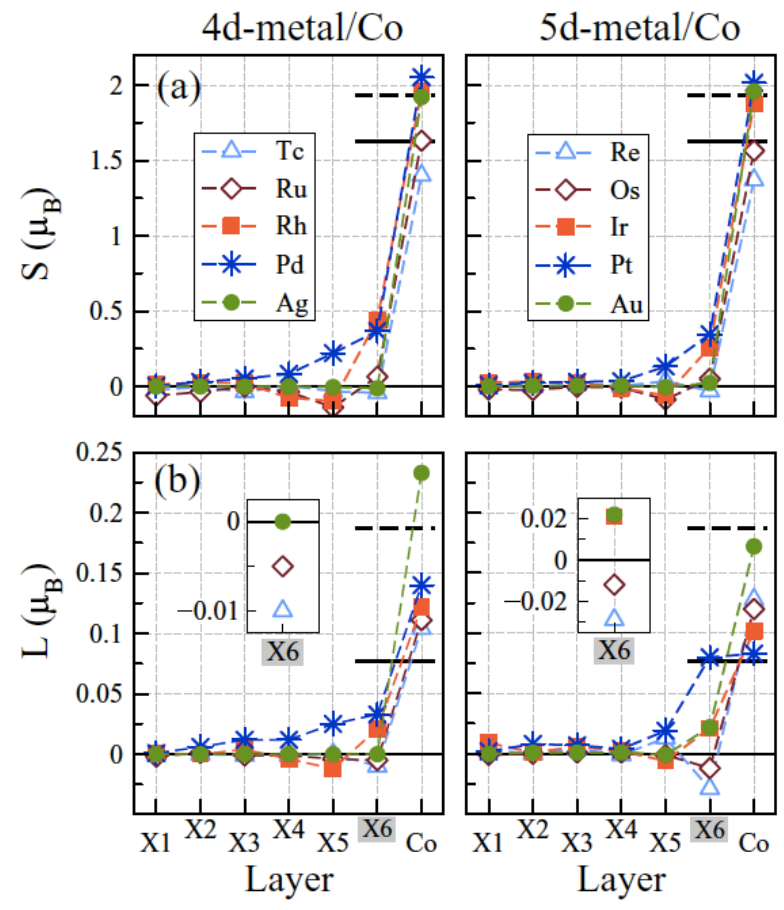

Figure 5: (Color online) Layer-resolved magnetic properties of the X/Co interfaces, where $\mathrm{X}$ is a heavy metal. (a) Spin and (b) orbital contributions to the magnetic moment for an in-plane magnetization. The left (right) panels represent the $4 \mathrm{~d}(5 \mathrm{~d})$ metals, as indicated in the figure. The solid (dashed) line indicates the value for the bulk (freestanding layer of) Co. From Ref. [22].

(ii) the orbital moment of the $3 d$ metal is enhanced. Metals with partially filled $d$ shells acquire a large magnetic moment $(\mathrm{Pt}, \mathrm{Pd}, \mathrm{Rh}$ and $\mathrm{Ir}$ ), which vanishes for metals with filled $d$ shells ( $\mathrm{Au}$ and $\mathrm{Ag}$ ), as reported on Fig. 5(a). The magnetic moment is very small in the case of $\mathrm{Tc}, \mathrm{Ru}, \mathrm{Os}$, and Re, but does not vanish. In addition, the transition metal interface acquires an induced orbital moment that is negative for $\mathrm{Tc}, \mathrm{Ru}, \mathrm{Re}$, and $\mathrm{Os}$, and positive at the interface with Rh, Pd, Ag, Ir, Pt, and Au, see Fig. 5(b).

\subsection{Interfacial spin-orbit coupling}

When an electron moves in an external electric field, or potential gradient, it experiences a magnetic field in its frame of motion, see Fig. 6(a). The coupling between this magnetic field and the spin degree of freedom (which is nothing but a Zeeman coupling) is the spin-orbit coupling given by $\hat{\mathcal{H}}_{\text {so }}=$ $(\xi / \hbar) \hat{\boldsymbol{\sigma}} \cdot(\boldsymbol{\nabla} \Phi \times \hat{\mathbf{p}})\left(\xi\right.$ is the spin-orbit coupling strength in $\left.\mathrm{eV} / \mathrm{m}^{2}\right)$. It couples the spin angular momentum $\hat{\boldsymbol{\sigma}}$ to the gradient of the crystal field $\nabla \Phi$. In crystals, the potential is composed of the spherical atomic potential and the rest of the potential composing the crystal field. It turns out that the potential 
(a)

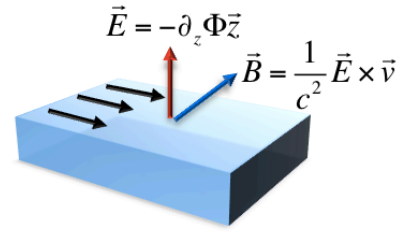

(c) Magnetic Metal

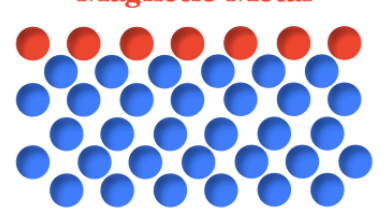

Heavy metal (b)

$\mathrm{E}(\mathrm{k})$

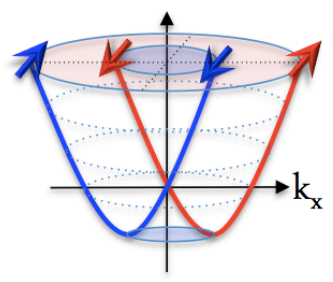

(d)

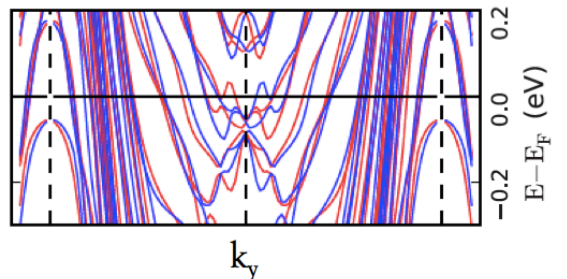

Figure 6: (a) Schematics of spin-orbit coupling: an electron flowing in a potential gradient $-\nabla \Phi$ experiences a magnetic field $\sim-\nabla \Phi \times \mathbf{v}$ in its frame of motion. (b) Two-dimensional band structure of the free electron Rashba gas. The red and blue arrows indicate the spin orientation. (c) Schematics of the transition metal interface. (d) Band structure of Co/Ir(111) interface computed from first principles, when the magnetization of Co lies in-plane along $+\mathbf{x}$ (red) and $-\mathbf{x}$ (blue). From Ref. [22].

In solid state, the potential $\Phi$ arises from the ionic environment of the electron, i.e. crystal structure, defects, impurities etc. Therefore, through spinorbit interaction, the information of this environment gets imprinted in the spin-dependent part of the wave function. In particular, in a solid possessing inversion symmetry $\left(\hat{\mathcal{H}}_{\mathbf{k}}=\hat{\mathcal{H}}_{-\mathbf{k}}\right)$ in the presence of spin-orbit coupling, Kramers degeneracy imposes that the two following Bloch states must be degenerate

$$
\begin{aligned}
|\mathbf{k}, n,+\rangle & =\left[u_{\mathbf{k}, n}^{\uparrow}|\uparrow\rangle+u_{\mathbf{k}, n}^{\downarrow}|\downarrow\rangle\right] e^{i \mathbf{k} \cdot \mathbf{r}}, \\
|\mathbf{k}, n,-\rangle & =\left[-u_{-\mathbf{k}, n}^{\downarrow *}|\uparrow\rangle+u_{-\mathbf{k}, n}^{\uparrow *}|\downarrow\rangle\right] e^{i \mathbf{k} \cdot \mathbf{r}} .
\end{aligned}
$$

As a consequence, neither the spin angular momentum nor the linear momentum are good quantum numbers anymore. The impact of spin-orbit coupling on the band structure of bulk $\mathrm{Pt}$ is reported in Fig. 7. The spin-orbit coupling removes the band degeneracy away from the $\Gamma$ point and induces energy splitting of the 
band structure. Because Pt has inversion symmetry, no splitting in momentum is observed.
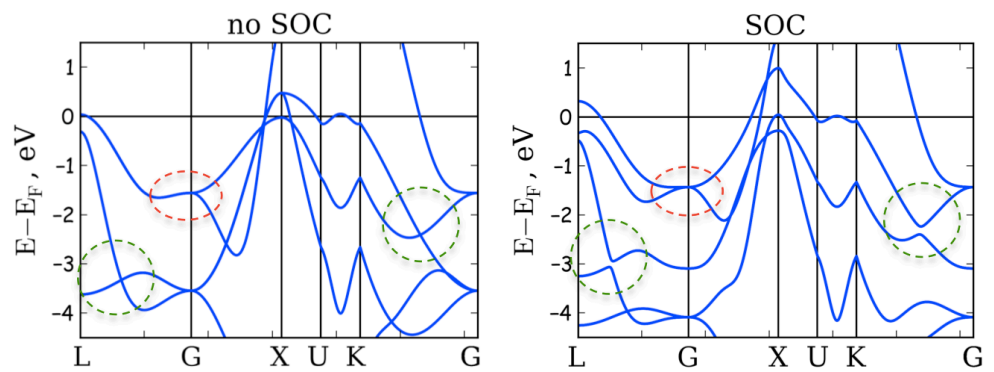

Figure 7: (Color online) Electronic band structure of bulk Pt in the absence (a) and in presence of spin-orbit coupling (b). The red circle shows that around the $\Gamma$ point, $\mathbf{k}=0$, no energy splitting is observed whereas away from it, the spin-orbit coupling induces energy splitting at the band-crossing points. Notice that the band structure is split only in energy, but not in momentum. (Courtesy from S. Grytsyuk)

What changes when going from the bulk to the interface? The usual way to look at the problem is by considering symmetry arguments. In such systems, the interface breaks the inversion symmetry along the normal to the interface, $\mathbf{z}$. Spatial inversion symmetry breaking allows the spin-orbit coupling to develop terms that are odd in momentum $\mathbf{k}$, which is well known since the pioneering work of Dresselhaus in bulk non-centrosymmetric semiconductors [27]. In the case of interfacial symmetry breaking, a simplistic picture suggests that the gradient of potential becomes $\langle\xi \nabla V\rangle \approx-\alpha \mathbf{z}$ and the spin-orbit coupling Hamiltonian reduces to $\hat{\mathcal{H}}_{\mathrm{R}} \approx-\alpha \hat{\boldsymbol{\sigma}} \cdot(\mathbf{z} \times \mathbf{k})$, where $\alpha$ is the so-called Rashba parameter, as originally proposed by Vas'ko [28] and studied by Bychkov and Rashba [29] in the context of low-doped two dimensional semiconducting quantum wells.

(a)

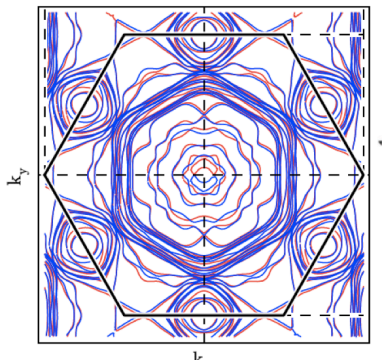

(b)

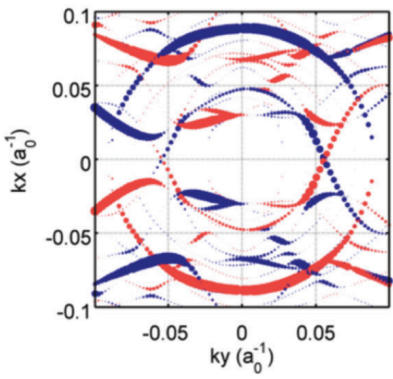

(c)

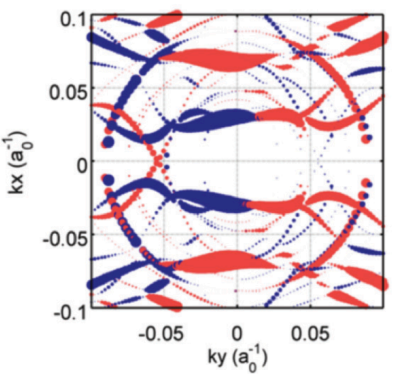

Figure 8: (a) Two-dimensional Fermi surface of $\operatorname{Ir} / \mathrm{Co}(111)$ interface in $\left(k_{x}, k_{y}\right)$ plane, when the magnetization direction is along $+\mathbf{x}$ (red) or $-\mathbf{x}$ (blue). The distortion of the Fermi surface reflects the coupling between spin and momentum degrees of freedom [22]. (b) The $x$-component and (c) $y$-component of spin at $\mathrm{Co} / \mathrm{Pt}(111)$ interface. The magnitude of each state's spin is proportional to the dot size, and blue dots refer to positive value of the spin component, while red dots refer to negative values [30]. 
Although this picture is very popular and widely used in the literature, the actual origin of Rashba spin-orbit coupling is a bit more subtle. As mentioned above, in a crystal the potential gradient is dominated by the spherical atomic potential, so that the largest contribution to spin-orbit coupling comes from the nuclei themselves rather than from the crystal field. In other words, spin-orbit coupling is essentially of the Russel-Saunders form, $\hat{\mathcal{H}}_{\text {so }}=(\xi / \hbar)\left\langle\partial_{r} \Phi / r\right\rangle \hat{\mathbf{L}} \cdot \hat{\boldsymbol{\sigma}}$. That being said, close to an interface the wave function experiences symmetry breaking, such that the potential gradient gets imprinted in the wave function itself, rather than in the spin-orbit coupling Hamiltonian. This aspect was emphasized in an inspiring study by Bihlmayer et al. [31]. In a bulk crystal, the eigenstates are given by Bloch states. Close to the nucleus, the Bloch states are well represented by spherical harmonics, i.e. $s, p$ and $d$ orbitals. Close to an interface, the potential gradient mixes these orbitals such that a state with a predominantly $l$ character hybridizes with states of $l \pm 1$ characters. In other words, $d$ and $p$ orbitals get mixed upon the potential gradient, such that the effective spin-orbit coupling, projected on the basis of the bulk Bloch states, becomes odd in momentum $k$. The sign of the potential gradient, and corresponding the charge imbalance around the nucleus, determines the sign of Rashba spin-orbit coupling [32, 33, 34, 35]. This results in quite a complex form of the band splitting. Indeed, while the initial Rashba model assumes a free electron gas with a circular Fermi surface, it is clear that transition metals display a much more complicated band structure, as illustrated on Fig. 8. However, in spite of this complexity, the overall physics associated with Rashba spin-orbit coupling is valid, as discussed in Section 4.
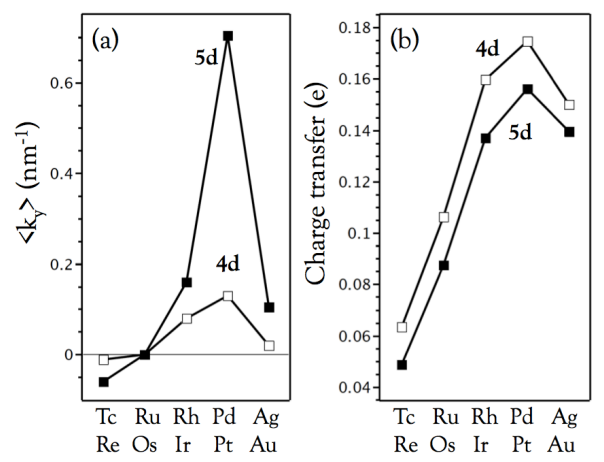

Figure 9: (a) Effective momentum shift $\left\langle k_{y}\right\rangle$ for $\mathrm{Co} / \mathrm{X}(111)$ interface and (b) corresponding charge transfer of the $d$-orbitals from the substrate to the Co overlayer. From [22].

In a recent work, Grytsyuk et al. [22] have systematically calculated the $k$ asymmetric spin-splitting occurring at the interface between Co and transition metal substrates. In their method, the authors compute the effective momentum shift of the Fermi surface upon magnetization reversal [see Fig. 8(a)]. The results are reported on Fig. 9(a). It is remarkable that the spin-splitting follows roughly the charge transfer between the substrate and the Co overlayer shown 
204 in Fig. 8(b), as well as the Stoner criterion reported on Fig. 2(a,b). Indeed, the $205 k$-asymmetric spin-splitting is driven by the $4 d$ or $5 d$ orbitals (which possess the 206 strongest spin-orbit coupling) and is therefore expected to be maximum with $207 \mathrm{Pd}$ and Pt substrates, respectively. 


\section{Dzyaloshinskii-Moriya Interaction}

In the previous section, we discussed the onset of $k$-asymmetric spin-splitting at transition metal interfaces. We showed that this effect emerges from the cooperation between spin-orbit coupling and interfacial symmetry breaking, and is very sensitive to interfacial orbital hybridization. At magnetic interfaces, the interfacial spin-momentum locking results in antisymmetric exchange interaction that can stabilize chiral magnetic textures.

\subsection{General principle}

Magnetic materials lacking inversion symmetry can host chiral magnetic objects whose dynamics has attracted significant attention lately [36]. Fundamentally, chiral magnetic objects arise from the competition between ferro- or antiferromagnetic exchange $\left(\sim J_{i j} \mathbf{S}_{i} \cdot \mathbf{S}_{j}\right)$ and a so-called antisymmetric exchange $\left(\sim \mathbf{D}_{i j} \cdot\left(\mathbf{S}_{i} \times \mathbf{S}_{j}\right)\right)[37,38]$. While the former tends to align neighboring spin in a collinear configuration, the latter tends to align neighboring spins perpendicular to each other, as illustrated on Fig. 10(a). As a result, spin-spirals or magnetic skyrmions can be achieved either as a ground state [39, 40, 41, 42, 43], or as metastable states [44, 45, 13, 14, 46, 47, 48]. An example of magnetic skyrmion is represented on Fig. 10(b). A crucial ingredient for the generation of such chiral textures is the Dzyaloshinskii-Moriya antisymmetric magnetic interaction (DMI) $[37,38]$ arising from spin-orbit coupling. Originally proposed in the context of Mott insulators [37, 38], weak metallic ferromagnets and spin glasses [49], it has recently been identified at transition metal interfaces, resulting in Néel domain walls $[50,51,52]$, spin spirals [53, 54, 55, 56], and skyrmions with a defined chirality $[43,57]$. In addition, DMI is known as the driving force for several magnetic phenomena such as spin glasses [49], magnon Hall effect [58], magnonic torque [59,60], multiferroicity [61], and chiral molecular magnets $[62,63]$.

We also emphasize that non-collinear magnetic textures can also emerge out of frustration [65]. For instance, even in the presence of inversion symmetry, when all the nearest neighbor exchange interactions cannot be fulfilled simultaneously, it is often preferable for the magnetic structure to adopt a non-collinear configuration, see Fig. 10(c). However, since inversion symmetry is conserved, the non-collinear magnetic system is not chiral, i.e. the ground state is degenerate and comprises multiple configurations that are energetically equivalent. An example is the $120^{\circ}$ spin spirals obtained in triangular lattices or the $3 \mathrm{Q}$ configuration obtained in $\mathrm{Mn} / \mathrm{Cu}(111)$ bilayers [64, 66], see Fig. 10(d). This topic remains out of the scope of the present discussion.

\subsection{Kashid's trimer model}

While the original theories of DMI apply in a context that is quite different from transition metal interfaces [38, 49], recent phenomenological models have explored the physics of DMI in metallic systems. Kim et al. [67] and Garate et al. [68] both proposed that Rashba or Dirac spin-orbit coupling in a two 
A

B

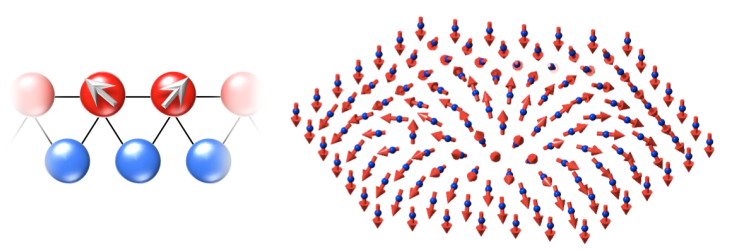

$\mathrm{C}$
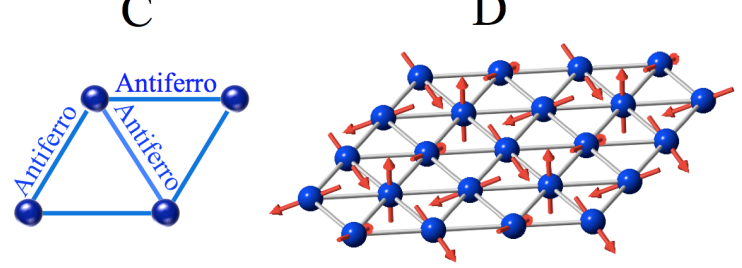

Figure 10: (a) The combination between spin-orbit coupling, magnetism and inversion symmetry breaking at transition metal interfaces gives rise to non-collinear magnetic textures, such as, but not limited to, magnetic skyrmions, displayed in panel (b). (c) Alternatively, certain frustrated systems involving the competition between ferromagnetic and antiferromagnetic exchange interactions support non-collinear magnetic textures, such as multiple-Q spin spirals. Panel (d) shows the 3Q texture obtained in $\mathrm{Mn} / \mathrm{Cu}(111)$ [64].

dimensional electron gas can result in DMI. In these theories, the magnetization is modeled by localized $d$-type electrons, while the transport is due to the $s$-type electrons. Hence, through Rashba or Dirac spin-orbit coupling, the itinerant electrons mediate an antisymmetric exchange between the local magnetic moments. In this limit, DMI is of the form $E_{\mathrm{DM}} \sim \mathbf{m} \cdot[(\mathbf{z} \times \boldsymbol{\nabla}) \times \mathbf{m}]$. In other words, at interfaces, one expects that DMI creates an effective magnetic field $\mathbf{H}_{\mathrm{DM}} \sim \mathbf{m} \times\left(\mathbf{z} \times \mathbf{e}_{i}\right)$, where $\mathbf{e}_{i}$ is the direction of the magnetic texture. For perpendicularly magnetized materials, such a magnetic field favors Néel walls over Bloch walls [69], as observed experimentally a transition metal interfaces $[50,51,52]$.

A finer "minimal" model was proposed by Kashid et al. [70]. In this approach, the authors consider a trimer, composed of two magnetic atoms (A and B) coupled to a non-magnetic atom (C) that possesses spin-orbit coupling [see Fig. 11(a)]. The magnetic atoms possess only $d_{x z}$ orbitals, while the nonmagnetic atom possesses both $d_{x z}$ and $d_{y z}$. The Hamiltonian of the trimer reads $\hat{\mathcal{H}}=\hat{\mathcal{H}}_{0}+\hat{\mathcal{H}}_{\mathrm{m}}+\hat{\mathcal{H}}_{\text {hop }}+\hat{\mathcal{H}}_{\text {so }}$, where

$$
\begin{aligned}
& \hat{\mathcal{H}}_{0}=\epsilon_{A} \hat{c}_{x z, A}^{\dagger} \hat{c}_{x z, A}+\epsilon_{B} \hat{c}_{x z, B}^{\dagger} \hat{c}_{x z, B}+\epsilon_{C}\left(\hat{c}_{x z, C}^{\dagger} \hat{c}_{x z, C}+\hat{c}_{y z, C}^{\dagger} \hat{c}_{y z, C}\right) \\
& \hat{\mathcal{H}}_{\mathrm{m}}=-\frac{J}{2}\left(\hat{c}_{x z, A}^{\dagger}\left(\hat{\boldsymbol{\sigma}} \cdot \mathbf{m}_{A}\right) \hat{c}_{x z, A}+\hat{c}_{x z, B}^{\dagger}\left(\hat{\boldsymbol{\sigma}} \cdot \mathbf{m}_{B}\right) \hat{c}_{x z, B}\right), \\
& \hat{\mathcal{H}}_{\mathrm{hop}}=t_{1}\left(\hat{c}_{x z, A}^{\dagger} \hat{c}_{x z, C}+\hat{c}_{x z, B}^{\dagger} \hat{c}_{x z, C}\right)+t_{2}\left(\hat{c}_{x z, A}^{\dagger} \hat{c}_{y z, C}-\hat{c}_{x z, B}^{\dagger} \hat{c}_{y z, C}\right)+c . c
\end{aligned}
$$

Here, $\hat{\mathcal{H}}_{0}$ is the unperturbed Hamiltonian of the three isolated atoms, $\hat{\mathcal{H}}_{\mathrm{m}}$ de- 
scribes the intrinsic magnetic coupling on atoms $\mathrm{A}$ and $\mathrm{B}$, where $\mathbf{m}_{A, B}=$ $(\cos \varphi, \pm \sin \varphi, 0)$ [70], and $\hat{\mathcal{H}}_{\text {hop }}$ describes the hopping between the three atoms. The hopping energies, $t_{1}$ and $t_{2}$, are defined within Slater-Koster parametrization [71],

$$
\begin{aligned}
& t_{1}=\cos ^{2} \theta V_{d d \pi}+\sin ^{2} \theta V_{d d \delta}, \\
& t_{2}=\sin \theta \cos \theta\left(V_{d d \pi}-V_{d d \delta}\right),
\end{aligned}
$$

where $V_{d d \pi}$ and $V_{d d \delta}$ are the Slater-Koster parameters and $\theta$ is the angle represented on Fig. 11(a). For simplicity, the direct hopping between A and B is discarded. Finally, the spin-orbit coupling is only active on the non-magnetic atom $\mathrm{C}$, and mixes orbitals $d_{x z}^{C}$ and $d_{y z}^{C}$,

$$
\hat{\mathcal{H}}_{\mathrm{so}}=\frac{i \xi}{2}\left(\left(\hat{c}_{x z, C}^{\uparrow}\right)^{\dagger} \hat{c}_{y z, C}^{\downarrow}-\left(\hat{c}_{y z, C}^{\uparrow}\right)^{\dagger} \hat{c}_{x z, C}^{\downarrow}\right)+c . c .
$$

Since DMI is, to the lowest order, linear in spin-orbit coupling, one can get an estimate of its strength by computing

$$
E_{\mathrm{DM}}=\int_{-\infty}^{\epsilon_{\mathrm{F}}} d \epsilon \operatorname{Tr}\left[\hat{\rho}_{0} \hat{\mathcal{H}}_{\mathrm{so}}\right],
$$

where $\hat{\rho}_{0}$ is the density matrix in the absence of spin-orbit coupling. By looking at the form of the spin-orbit coupling term, Eq. (8), one realizes that in order to obtain large DMI, the unperturbed eigenstate should possess both $d_{x z}$ and $d_{y z}$ characters with opposite spins. As a result, DMI stems from (i) non-collinear magnetism (both up and down spins are present), as well as (ii) symmetry breaking (that mixes $d_{x z}$ and $d_{y z}$ orbitals). The former is obtained by taking $\varphi \neq 0$, while the latter requires $t_{2} \neq 0$. Moreover, condition (i) implies that half metals (where only one spin species is present at Fermi energy) are unlikely to show DMI. In summary, in this model $E_{\mathrm{DM}}$ is very sensitive to the hybridization between the spin-mixed orbitals, $d_{x z, A(B)}$, and the spin-orbit coupled orbitals, $d_{x z, C}$ and $d_{y z, C}$.

Let us now explore the outcomes of this model. For the calculation, we take the same parameters as in Ref. [70]: $V_{d d \pi}=0.8 \mathrm{eV}, V_{d d \delta}=-0.07 \mathrm{eV}, \theta=\pi / 3$, $\epsilon_{A}=\epsilon_{B}=0, J=1.125 \mathrm{eV}$, and the Fermi energy is $\epsilon_{\mathrm{F}}=1.14 \mathrm{eV}$. We also take a canted magnetic configuration by setting $\varphi=\pi / 4$, which provides the maximum DMI energy. Finally, we take an energy broadening $\Gamma=0.025 \mathrm{eV}$ to perform the energy integral. The spin- and orbital-resolved density of states are reported on Fig 11(b)-(e) when modifying the relative energy shift $\epsilon_{C}-\epsilon_{A}$. Three main situations can be obtained. When $\epsilon_{C}-\epsilon_{A}=0$, the non-magnetic orbitals are located in-between the majority and minority states of the magnetic atoms such that their overlap remains weak [Fig 11(b)]. When lowering the onsite energy of the non-magnetic orbitals, they get closer to Fermi level and approach the energy level of the minority spins, such that their overlap increases [Fig 11(c,d)]. When exceeding $\epsilon_{C}-\epsilon_{A} \approx 1 \mathrm{eV}$, the non-magnetic orbitals are pushed above the Fermi energy and the orbitals of the magnetic ions remain mostly unperturbed [Fig 11(d)]. 
A
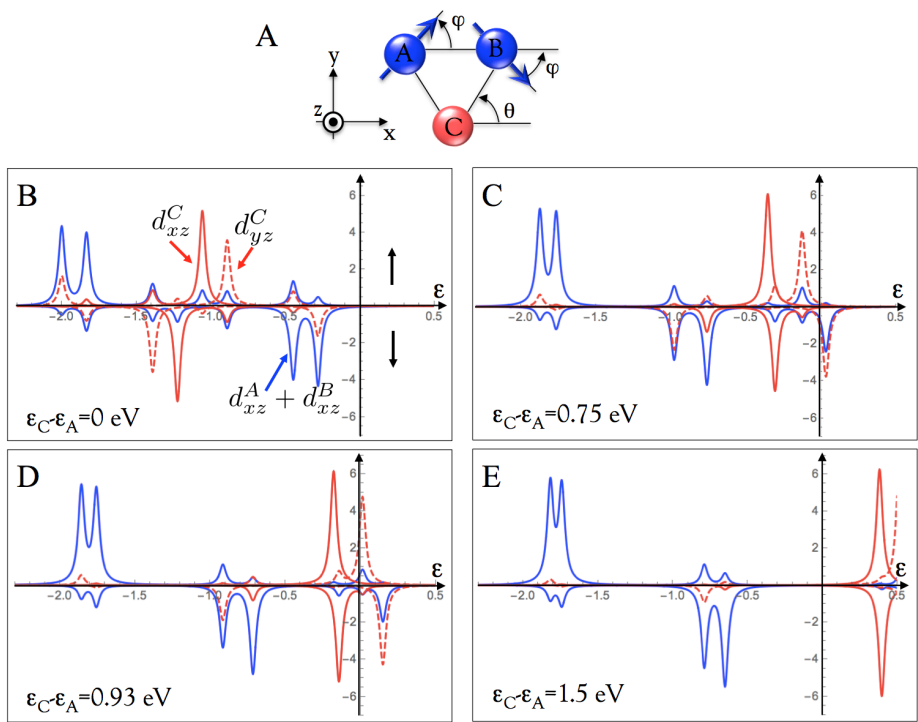

Figure 11: (a) Schematics of Kashid's trimer model. (b)-(e) Orbital-resolved density of states for different relative energy shift between the magnetic and non-magnetic orbitals. The blue lines refer to the magnetic orbitals $d_{x z, A}+d_{x z, B}$, the solid red lines correspond to the nonmagnetic orbital $d_{x z, C}$ and the dashed red lines to $d_{y z, C}$. The parameters are given in the text.

Figure 12(a) displays the DM energy $E_{\mathrm{DM}}$ as a function of the relative energy shift, $\epsilon_{C}-\epsilon_{A}$. The vertical lines correspond to the cases reported on Fig. $11(\mathrm{c}, \mathrm{d}, \mathrm{e}) . E_{\mathrm{DM}}$ remains vanishingly small as long as the non-magnetic orbital remain in-between the magnetic orbitals, $\epsilon_{C}-\epsilon_{A}<0.5 \mathrm{eV}$ [Fig. 11(a)], then increases moderately when the non-magnetic orbital start overlapping with the magnetic ones, close to Fermi energy [Fig. 11(b)]. When the non-magnetic orbital enters in resonance with the minority bands, around $0.9 \mathrm{eV}$, a transition occurs from positive to negative DMI. It reverses back to positive DMI when further lowering the non-magnetic orbital energy and eventually vanishes when the non-magnetic orbitals are expelled above Fermi level. The change of sign of DMI can be analyzed by tracking the density of states of $d_{y z, C}$ at Fermi energy. As explained above, since DMI scales with $d_{x z, C^{-}} d_{y z, C}$ orbital mixing, it depends on the amount majority versus minority $d_{y z, C}$ orbitals at Fermi level. This quantity is very sensitive to the details of the orbital hybridization, as shown in these calculations.

Since the physics of orbital hybridization is quite complex, it is reasonable to seek for a macroscopic quantity that correlates with $E_{\mathrm{DM}}$. Let us define two such quantities, the induced magnetic momentum on the non-magnetic ion $m_{C}$ 

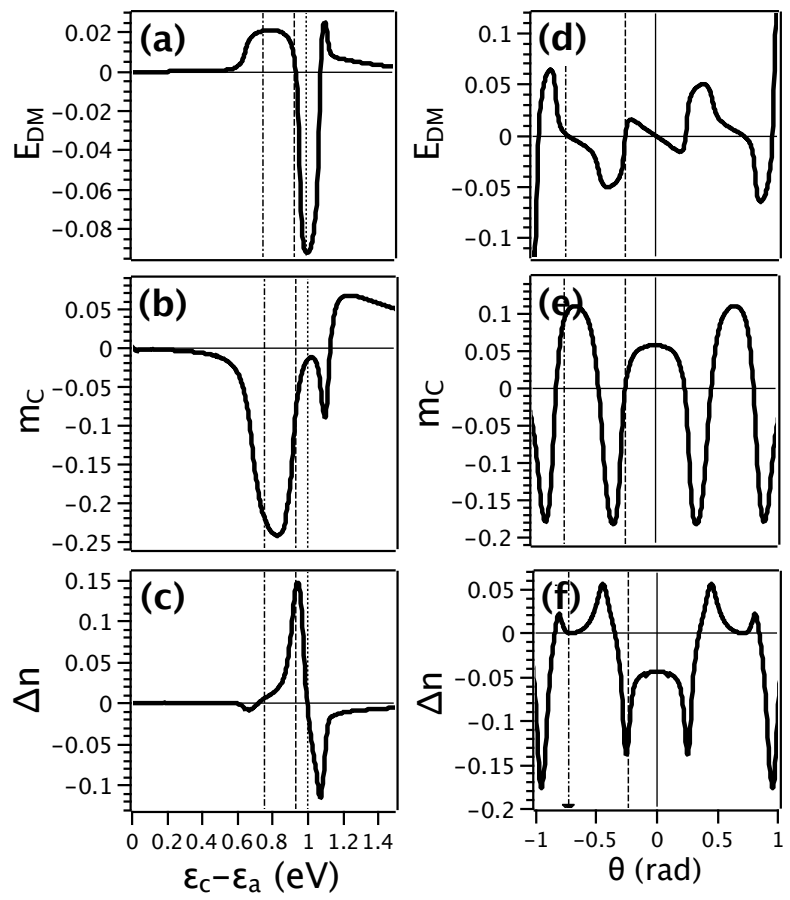

Figure 12: (a) Dependence of DMI energy $E_{\mathrm{DM}}$, (b) induced magnetization $m_{C}$ and (c) charge transfer $\Delta n$ as a function of the relative energy shift between the magnetic and non-magnetic orbitals. The vertical dashed-dotted, dashed and dotted lines refer to $\epsilon_{C}-\epsilon_{A}=0.75 \mathrm{eV}$, $\epsilon_{C}-\epsilon_{A}=0.93 \mathrm{eV}$ and $\epsilon_{C}-\epsilon_{A}=1 \mathrm{eV}$, respectively. (d) Dependence of DMI energy $E_{\mathrm{DM}}$, (e) induced magnetization $m_{C}$ and (f) charge transfer $\Delta n$ as a function of the angle $\theta$, as defined in Fig. 11. The vertical dashed-dotted, dashed and dotted lines refer to three noticeable situation discussed in the text.

and the charge transfer between the magnetic and non-magnetic ions $\Delta n$,

$$
\begin{aligned}
m_{C} & =\int_{-\infty}^{\epsilon_{\mathrm{F}}} d \epsilon \operatorname{Tr}\left[\hat{\rho}_{0}^{C} \hat{\sigma}_{z}\right], \\
\Delta n & =\int_{-\infty}^{\epsilon_{\mathrm{F}}} d \epsilon \operatorname{Tr}\left[\hat{\rho}_{0}^{A}+\hat{\rho}_{0}^{B}-\hat{\rho}_{0}^{C}\right] .
\end{aligned}
$$

These two quantities are reported on Fig. 12(b) and (c). We observe two remarkable features. First both positive maxima of $E_{\mathrm{DM}}$ at $\epsilon_{C}-\epsilon_{A} \approx 0.8 \mathrm{eV}$ and $1.08 \mathrm{eV}$ correspond to a negative maximum in induced magnetization. Second, the largest $E_{\mathrm{DM}}$ value is reached around $\epsilon_{C}-\epsilon_{A} \approx 0.93$, which corresponds to a local minimum in $m_{C}$ and a vanishing $\Delta n$. What we wish to emphasize here is that there is no straightforward, systematic correlation between $E_{\mathrm{DM}}$ and either induced magnetization or charge imbalance, even in this simple model.

Another interesting study is to vary the symmetry breaking. To do so, we simply vary the angle $\theta$ in the range $\pm \pi / 3$, assuming that the overlap integrals, 
$V_{d d \pi}$ and $V_{d d \delta}$ remain unmodified. The results for $E_{\mathrm{DM}}, m_{C}$ and $\Delta n$ are reported on Fig. 12(d)-(f). The DMI is clearly antisymmetric, while the induced magnetism and charge imbalance remain symmetric. Again, a correlation between these three quantities can be found for some specific points [denoted by the vertical lines in Fig. 12(d)-(f)], but no systematic rule of thumb can be determined, highlighting the complexity of the phenomena involved.

The analysis of Kashid's trimer model reveals the high sensitivity of DMI to the specific orbital hybridization scheme present at interfaces. As such, one would expect an even more complex behavior when performing multiple-orbital calculations. That being said, since the evolution of the $d$-bands shows some systematic trends across the transition metal series, as discussed in details in Section 2, one could optimistically hope that such trends reflect in the magnitude of DMI.

\subsection{First principles methods}

In density function theory, DMI energy can be estimated by calculating the energy of the magnetic system in the presence of a spin spiral. Indeed, the impact of DMI on spin spirals resembles the influence of Rashba coupling on itinerant electrons: it distorts their energy dispersion [72]. The momentum shift of this dispersion provides a measurement of DMI. The main technical difficulty when using density functional theory is to accurately evaluate the energy of the system in the presence of both spin-spiral (i.e. non-collinear magnetism) and spin-orbit coupling (the spin is not a good quantum number anymore). Indeed, building a spin-spiral in real space is restricted to short wave lengths (typically less than 10 atoms [73]) and becomes computationally prohibitive in the longwavelength limit. This goes without mentioning the specific issues related to the application of the local magnetic field constraining the spin direction [73]. An alternative powerful approach employs the generalized Bloch theorem [74, 75] that allows studying the spin spiral in the reciprocal space [76]. In that case, the DMI energy can be calculated for the whole range of spin-spiral wavelengths, from $q=0$ (ferromagnetic) to $q=\pi / a$ (antiferromagnetic), up to the first order perturbation in spin-orbit coupling $[77,78]$. Here $a$ is the lattice constant of the crystal unit cell. This is the method we will be using below.

Besides the generalized Bloch theorem and constrained spin-spiral methods, two other approaches have been proposed lately. Freimuth et al. [79, 80] developed a linear response theory that provides an estimation of the DMI in the limit $q \rightarrow 0$. The authors demonstrated that at $0 \mathrm{~K}$, DMI can be written

$$
D_{i j}=\frac{\hbar}{2 \pi \Omega} \Re \int d \epsilon f(\epsilon)\left(\epsilon-\epsilon_{\mathrm{F}}\right) \operatorname{Tr}\left[\mathcal{T}_{i} \hat{G}^{R} \hat{v}_{j} \partial_{\epsilon} \hat{G}^{R}-\mathcal{T}_{i} \partial_{\epsilon} \hat{G}^{R} \hat{v}_{j} \hat{G}^{R}\right],
$$

where $\hat{\mathbf{v}}$ is the velocity operator, $\hat{G}^{R}=\left(\epsilon-\hat{\mathcal{H}}-\hat{\Sigma}^{R}\right)^{-1}$ is the retarded perturbed Green's function of the system $\left(\hat{\Sigma}^{R}\right.$ being the retarded self-energy including disorder etc.), $f(\epsilon)$ is Fermi-Dirac distribution, $\Omega$ is the volume of the unit cell, $\Re$ takes the real part and $\operatorname{Tr}$ denotes the trace. Here $\mathcal{T}=\mu_{\mathrm{B}} \mathbf{B}^{\mathrm{x}} \times \hat{\boldsymbol{\sigma}}$, is the torque operator between the electron spin and the exchange field, $\mu_{\mathrm{B}}$ is 
Bohr's magneton. In this definition, $D_{i j}$ is in $\mathrm{eV} \cdot \mathrm{m}^{-2}$. This formula involves the summation over all the states located below Fermi level. Physically, Eq. (12) describes the Fermi sea contribution of an equilibrium torque $\mathcal{T}$ induced by electrons flowing with a velocity $\hat{\mathbf{v}}$. A similar physical picture has been proposed by Kikuchi et al. [81], where spin-orbit coupling enables the flow of an equilibrium spin current that interacts with spin spirals and induces a "Doppler shift", corresponding to the DMI. In Kikuchi's picture, the problem boils done to calculating the equilibrium spin current $\mathcal{J}_{s}$ that interacts with the magnetic texture through the DM energy term

$$
\mathcal{H}_{\mathrm{DM}}=(\hbar / 2) \int_{\Omega} \mathbf{m} \cdot\left[\left(\mathcal{J}_{s} \cdot \nabla\right) \times \mathbf{m}\right] d^{3} \mathbf{r},
$$

where $\mathcal{J}_{s}$ is a $3 \times 3$ tensor in the spin $\otimes$ real space. Notice that in the above expression, the projection $\mathcal{J}_{s} \cdot \nabla$ is taken on the real space coordinates, such that this term is a 3 -component vector.

\subsection{DMI at $3 d / 5 d$ interfaces}

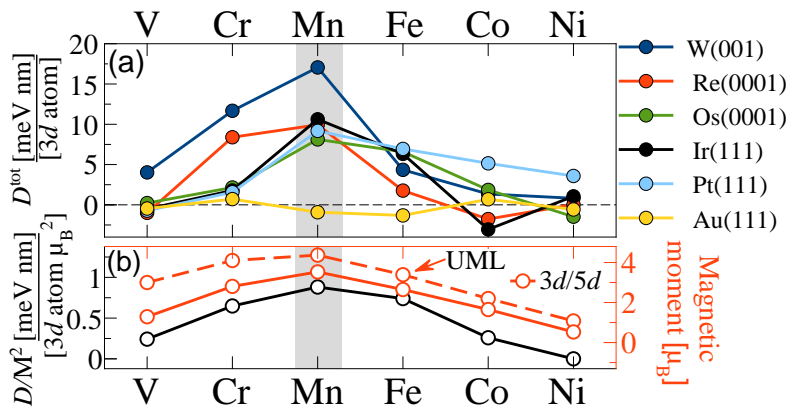

Figure 13: (a) Strength and sign of Dzyaloshinskii-Moriya interaction (DMI) $D^{\text {tot }}$ in $3 d$ transition metal monolayers on $5 d$ substrates calculated around their magnetic ground state combining the relativistic spin-orbit coupling effect with spin spirals. A positive sign of $D^{\text {tot }}$ indicates

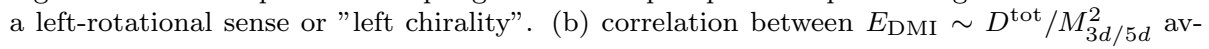
eraged over $3 d / 5 d$ interfaces (black line) versus the $3 d$ overlayer, the magnetic moments in $3 d$ transition metal unsupported monolayer (UML - dashed red line), and the local magnetic moment per atom averaged over $3 d / 5 d$ interfaces (solid red line).

There are many reports on the spin textures in $3 d / 5 d$ multilayered material stacks and interfaces $[82,53,54,43,55,56]$, and several models have been developed to explain the physics of DMI $[77,78,70,73,83,84]$. In spite of this intense effort, the various aspects of the physics underlying the onset of DMI and its behavior are not well understood. More specifically, the DMI in $3 d / 5 d$ interfaces depends sensitively on the details of the electronic structure, in which the high complexity of interfacial hybridization hinders the development of qualitative and quantitative predictions in these material combinations. In this regard, controlling the sign and magnitude of DMI represents an outstanding challenge 
for modern magnetism. For this reason, a universal phenomenological description of the DMI in transition-metal interfaces is required to simplify the complex physical picture, and can provide significant guidance for designing new interfaces and especially controlling their spin textures. From a fundamental point of view, we believe that predicting the general trend is extremely important to understand the underlying physical mechanisms of DMI. Therefore, it is instructive to develop a new physical picture by predicting the chemical trend of DMI across the majority of $3 d / 5 d$ interfaces. In this Section, we review a systematic investigation of DMI for $3 d$ metals $(\mathrm{V}, \mathrm{Cr}, \mathrm{Mn}, \mathrm{Fe}, \mathrm{Co}, \mathrm{Ni}$ ) as overlayers on $5 d$ substrates (W, Re, Os, Ir, Pt, Au) [21]. The calculations are performed using density functional theory within the local density approximation to the exchange correlation functional [85], using the full potential linearized augmented plane wave method in film geometry [86] as implemented in the Jülich density functional theory code FLEUR [87]. The DMI energy is computed to the first order in spin-orbit coupling within the framework of the generalized Bloch theorem $[64,78]$.

In Fig. 13 we summarize our results of total DMI energy $D^{\text {tot }}$ as a function of the $3 d$ overlayer element for various $5 d$ substrates. According to our calculations, regardless of the sign found in $\mathrm{Co} / \mathrm{Re}(0001)$ and $\mathrm{Co} / \operatorname{Ir}(111)$ interfaces, the DMI shows a clear variation with respect to the $3 d$ filling where the Mn overlayer has the largest values and it gradually decreases for chemical elements on both sides of $\mathrm{Mn}$ in the $3 d$ transition metal row of the periodic table. This variation is actually very surprising since it reveals an interesting chemical trend, in which the DMI across the $3 d / 5 d$ interfaces clearly follows Hund's first rule [88] with a tendency similar to their magnetic trends in either the unsupported monolayers or ultrathin films [see Fig. 13(a)] [21]. Since the energy term resulting from this interaction is proportional to $M^{2}$, this correlation holds true and remains unchanged when considering the ratio of DMI divided by the square of the magnetic moment $M_{3 d / 5 d}^{2}$ for all studied $3 d / 5 d$ interfaces [see Fig. 13(b)]. Indeed, this appears consistent with the behavior observed in low-dimensional systems whose spin moments as a function of the number of $d$ electrons are well described by Hund's first rule [88, 20, 19] as shown in Fig. 13(b). The only exception is $3 d / \mathrm{Au}(111)$ systems where the magnitude of DMI is very small, independent of the $3 d$ overlayer. Despite the large spin-orbit coupling of $\mathrm{Au}$ compared with all $5 d$ substrates, the weak DMI seems unusual but can be understood on the basis of its electronic configuration, in which the $5 d$ shells are completely occupied [see Figs. 3 and 13(a)]. The latter indicates that the DMI depends critically not only on spin-orbit coupling and breaking of the inversion symmetry, but also on the $5 d$ wave function localization of the chosen substrate. It is also worthwhile to note that most of $3 d / 5 d$ interfaces have a positive sign of DMI, such that the left- or right-rotating character of the spin spiral depends on their ferro- or antiferromagnetic ground state [see Fig. 13(a)] [21].

These remarkable findings reveal the existence of magnetically correlated behavior between the DMI and spin magnetic moment. Furthermore, this trend was not expected since the other magnetic interactions, magnetic anisotropy energy and Heisenberg exchange interaction, behave differently and show a com- 
pletely different chemical trend when moving along the $3 d$ elements [18]. More specifically, although the magnetic anisotropy contributes to the magnetic energies, it does not show a positive correlation with magnetic moment of $3 d / 5 d$ thin films. For instance, Mn systems have the smallest magnetic anisotropies but the largest magnetic moment. In the same way, the Heisenberg-type exchange interaction parameter $J$ is also independent of $M$, being smaller for Mn than for Co. Obviously, the nearest-neighbor exchange interaction $\left(\mathrm{J}_{1}\right)$ for $3 d$ transition metals follows perfectly the Bethe-Slater curve and not Hund's first rule [89]. Further, this fact is also surprising as it is opposite to what is expected from the knowledge of magnetism in bulk and thin films. For instance, such uncorrelated behavior has also been recently observed in the skyrmion phase of $\mathrm{Mn}_{1-x} \mathrm{~F}_{x} \mathrm{Ge}$ by Gayles et al. [83], where the strength of DMI does not show any correlation with the magnetic moment of the $3 d$ transition metal. This provides additional evidence for a violation of direct relation of magnetic moment and DMI in alloy systems.

Since the relation between interfacial DMI and local magnetic moments of $5 d$ substrate has been so controversially debated lately, we emphasize here that the DMI shows no direct correlation with the proximity induced magnetism in $5 d$ across the $3 d / 5 d$ interfaces [21]. Indeed, the $5 d$ states are degenerate and partially occupied due the strong crystal-field splitting or large bandwidth, irrespective of deposited $3 d$ overlayer on the top, in good agreement with the experimental observations [90] and previous theoretical studies [18, 73]. This result is in contrast, however, with recent experiment by Ryu et al. [91] who claim that DMI should scale with the induced magnetization of the heavy metal.

The above discussion demonstrates that varying the orbital hybridization between the $3 d$ overlayer and the $5 d$ substrate impacts the DMI and the other magnetic interactions differently in those systems. More specifically, since the DMI emerges from a complex interplay between (i) the degree of spin polarization of the $3 d / 5 d$ interface atoms and their band filling, (ii) the strength of spin-orbit coupling in the underlying heavy metal $5 d$ substrate, and (iii) the inversion symmetry breaking at the interface, one does not necessarily expect a direct correlation between the magnetism of the $3 d$ overlayer and the DMI. This lack of correlation confirms our previous conclusions based on Kashid's model. In the following, we will explain in more detail the physical reasons behind the unexpected trend, pointing out the importance of this magnetic correlation from the electronic point of view.

The microscopic origin of the correlation displayed in Fig. 13 clearly involves the impact of the $3 d$-orbital magnetization and their electron filling on DMI, which, however, affects significantly the overlayer-substrate hybridization and consequently the magnetic coupling between the $3 d$ atoms in the monolayer plane. This can be understood by examining the energetic positions of $3 d / 5 d$ states and their spin-flip mixing processes. Indeed, as explained in the previous Section, we remind that the antisymmetric exchange mechanism, as an excitation signature, requires spin-flip transitions between occupied and unoccupied states through spin-orbit active $5 d$ states. Of course, this requires certain level of hybridization with the $3 d$ orbitals, since the energetic band alignment of the 
$3 d-5 d$ interface states around the Fermi energy controls the magnetic coupling of deposited $3 d$ atoms and, consequently, the DMI.

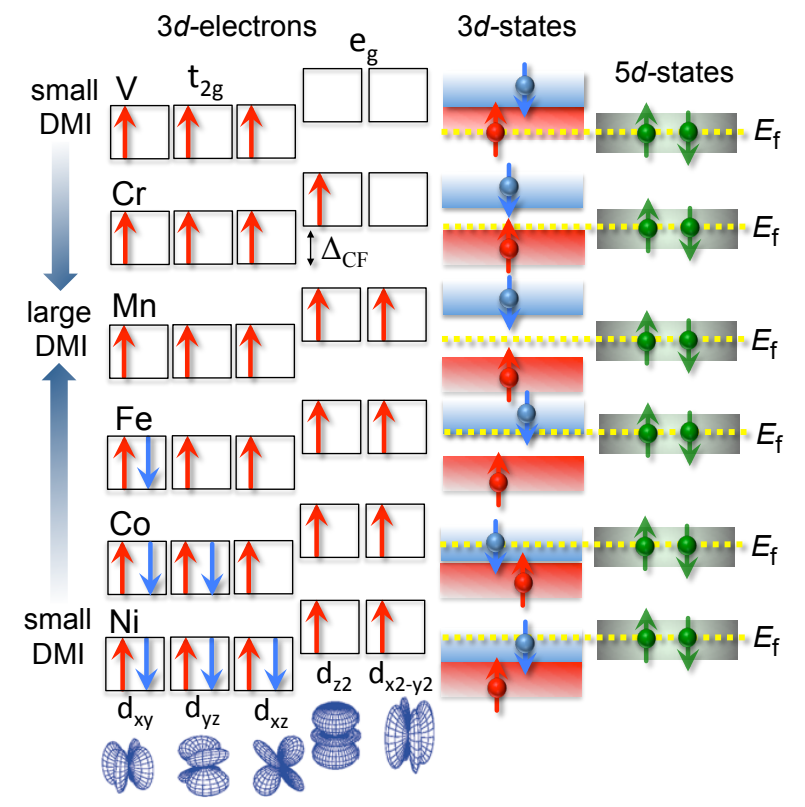

Figure 14: Left side: filling with electrons of $3 d$ transition metal elements into the five $3 d-$ orbitals according to Hund's first rule, spin-up and -down are shown by red and blue arrows, respectively. On the right side we show the spin-split band positions of $3 d$ states with respect to $5 d$-W states. Note, since the $5 d$ bandwidth is significantly larger than the crystal field splitting the $5 d$ states are degenerate at the Fermi level. $\Delta_{\mathrm{CF}}$ indicates the crystal-field splitting between the $t_{2 \mathrm{~g}}$ and $e_{\mathrm{g}}$ shells. From [21].

This fact is reflected, for instance, by the electronic configurations of the $3 d$ orbitals and their spin-split band positions with respect to $5 d \mathrm{~W}$ states, see Fig. 14. Inspecting this figure in more detail, one can see that the band lineups of $3 d$ and $5 d$ states reveal two interesting features, which are essentially similar to a first approximation. First, the $\mathrm{W}$ surface is weakly polarized where the $5 d$ states are degenerate and partially filled, consistent with the fact that those configurations of the outer electrons shell are dominated by the significant large bandwidth or by the crystal-field splitting. Note that the number of available $5 d$ states near the Fermi level and their bandwidth are fixed for a certain substrate depending on their filling. Second, the electron filling of the $3 d$ orbitals, according to Hund's first rule, fits well the band alignment of $3 d$ states around the Fermi level, as shown in Fig. 14. At this stage, it is important to stress out that the $3 d-5 d$ majority and minority spin states and their exchange-splitting gap are energetically aligned according to their calculated band structure, as depicted on Fig. 14, right side. The latter is found to be consistent with the electronic occupation of $3 d-5 d$ (in first approximation) spin channels (Fig. 14, left side) and thus obeying both crystal field splitting and Hund's first rule. 
In this context, since the $5 d$ bandwidth is significantly larger than the crystalfield splitting and the $5 d$ states are weakly polarized (degenerate and partially filled), the overall physics of the predicted trend in Fig. 13 is mostly governed by the band lineup of $3 d$ spin channels, themselves determined by Hund's first rule. Therefore, in a simple picture one can easily explain this fact for all $3 d$ elements.

By comparing the early and late $3 d$ overlayers, $\mathrm{V}$ and $\mathrm{Ni}$ respectively, one can observe that both spin channels are mostly either occupied or unoccupied; their band alignment with respect $5 d$ states is energetically unfavorable and, consequently, the spin-flip excitation processes are not allowed. As a result, the DMI is weak in both cases since this mechanism requires the availability of $3 d$ electrons, occupied and unoccupied, to facilitate the spin-flip process which is crucial for the appearance of the DMI. Proceeding from Ni to Co overlayer, the DMI remains relatively weak because of the small amount of unoccupied minority $3 d$ states. Keep in mind that the degeneracy of $5 d$ spin channels is not lifted by the presence of any $3 d$ overlayer on the top, despite the overlap of the relatively more extended $5 d$ wave function of the $\mathrm{W}$ substrate with the wave functions of $3 d$ overlayers. In other words, since the $5 d$ orbitals are more extended than $3 d$ ones, these orbitals are only weakly perturbed by the adsorption of $3 d$ elements. On the other hand, since the DMI is weaker it is more sensitive to the detail of the orbital hybridization between the substrate and the overlayer, resulting in a change of handedness of the magnetic structure. This actually happens for $\mathrm{Ni} / 5 d$ and $\mathrm{V} / 5 d$ interfaces on both sides of this trend, as well as for Co overlayer. This also holds true for Au substrate, because the $5 d$ states lie far below the Fermi level (the bonding character is less clear for the involved states) the DMI is weaker and therefore shows variations in the sign as a function of the $3 d$ overlayer (Fig. 13, yellow line). Such an oscillation in sign has been analyzed extensively for $\mathrm{Mn}_{1-x} \mathrm{~F}_{x}$ Ge by Gayles et al. [83] and has also been identified in the trimer model developed by Kashid et al. [70], where the sign and magnitude of DMI depend on the shifts of the single-particle energies with respect to the scalar-relativistic eigenvalues due to spin-orbit coupling $\left(\delta \epsilon_{\mathrm{k} \nu}=\delta \epsilon_{\mathrm{k} \nu}^{\mathrm{SOC}}-\delta \epsilon_{\mathrm{k} \nu}^{\mathrm{SR}}\right)$ in the energy range around the Fermi level [70]. According to perturbation theory, the $5 d$ orbitals "spin-orbit active states" are responsible for the spin-orbit coupling matrix elements $\mathcal{H}_{\mathrm{so}}(\xi \mathrm{L})$ and make essential contributions to DMI [see Fig. 6(a)]. However, despite the weak spin-orbit coupling in the $3 d$ overlayer their intra-atomic exchange field can easily modify the electronic structure around the Fermi energy and consequently change the strength of the DMI. One may conclude that the spin-orbit-induced energy shift of the highest occupied orbital depends sensitively on the charge rearrangement between the $3 d$ orbitals of opposite spin and, hence, Hund's first rule. In the following, we will demonstrate how the spin-flip excitations through spin-orbit coupling in half-filled or high spin $3 d$ overlayer make the largest contribution to the DMI.

The situation is completely different when moving to half-filled $3 d$ transition metals. In the case of Mn overlayer, the filling of the five Mn $3 d$ orbitals adopts a stable "high spin state" due to the small crystal field splitting between the 
$t_{2 g}$ and $e_{g}$ shells and, hence, half-band filling occur (see Fig. 14). The spinup (spin-down) channels are entirely occupied (unoccupied) and all transitions contribute to the DMI through the intermediate spin-orbit active $5 d$ states. In other words, the $3 d-5 d-3 d$ electron hopping is facilitated, resulting in a large DMI [92]. Therefore, the $\mathrm{Mn} / 5 d$ interfaces, in particular Mn/W(001), shows the largest magnetic antisymmetric exchange. When the DMI is strong enough to compete against the Heisenberg exchange and magnetic energy anisotropy, complex spin textures are more likely to appear. The latter holds true especially since the Heisenberg-type exchange interaction is independent of the magnetic moment $M$, and is smaller for Mn than for Co according to the Bethe-Slater curve. This result is in good agreement with the experimental observation of Bode et al. [53], who demonstrated for the first time that the strong DMI in Mn monolayer on a W(110) substrate can stabilize long-range spin spirals. Also, by combining spin polarized scanning tunneling microscopy and ab-initio theory, a unique rotational sense as a left-rotating cycloidal spin spiral was also found by Ferriani et al. [54].

The situation is almost similar for the half-filled $\mathrm{Fe}$ and $\mathrm{Cr}$ atoms but the exchange splitting is reduced, where most of the Fe spin-down ( $\mathrm{Cr}$ spin-up) states are still unoccupied (occupied). This fact clearly explains the sensitivity of the DMI to the choice of the $3 d$ overlayer and the degree of hybridization with $5 d$ states (see, e.g.,[93]). Recently, a similar trend for Mn, Co and Ni chains on $\mathrm{Pt}(111)$ step edge was found by Schweflinghaus et al. [92]. Concerning the sign of $D$ in the half-filled $3 d$ shells (Fe, Mn, Cr), the DMI prefers to maintain its left-hand chirality (left rotating). In general, although some subtleties can be observed when the DMI is small as discussed above, the atomic Hund's first rule overall gives a clear trend of the overlayer dependence of the DMI.

In order to further understand the chemical trend of DMI across the $3 d / 5 d$ interfaces, it is instructive to consider the charge transfer and induced dipole moment since the interfacial orbital hybridization in those systems is quite different in term of their bonding character. For the sake of clarity, we focus on $3 d$ MLs on $\mathrm{Au}(111)$ and $\mathrm{W}(001)$ as the two extreme cases on both sides of the trend. We first consider the charge density difference computed in the following manner: $\Delta \rho(\mathbf{r})=\rho_{\text {tot }}(\mathbf{r})-\left[\rho_{5 \mathrm{~d}}(\mathbf{r})+\rho_{3 \mathrm{~d}}(\mathbf{r})\right]$, where $\rho_{\text {tot }}(\mathbf{r}), \rho_{5 \mathrm{~d}}(\mathbf{r})$, and $\rho_{3 \mathrm{~d}}(\mathbf{r})$ are the charge density distributions of the conjugate system, W or Au substrate, and $3 d$ overlayer, respectively, each in the precise position they adopt in the adsorption configuration. The dipole moment $\mu(z)$ induced by $3 d$ adsorption can be obtained by integrating the half-cell volume along the $z$-direction $\mu(z)=\frac{1}{n} \int_{-c}^{-c+z / 2} z^{\prime} \Delta \bar{\rho}\left(z^{\prime}\right) d z^{\prime}$, where $z / 2$ is half the length of the supercell, $c$ is the distance from the tompost layer to the middle of the slab, and $n$ is the number of $3 d$ adatoms per unit cell. Note that the planar averaged charge density difference $\Delta \bar{\rho}\left(z^{\prime}\right)$ is $\Delta \rho(\mathbf{r})$ integrated over lateral coordinates $x$ and $y$ for each $z$ plane.

The DMI and the corresponding dipole moment values for $3 d / \mathrm{Au}(111)$ and $3 d / \mathrm{W}(001)$ are reported in Fig. 15. We find a pronounced correlation between the electric dipole moment and DMI in both cases, indicating that the physical 


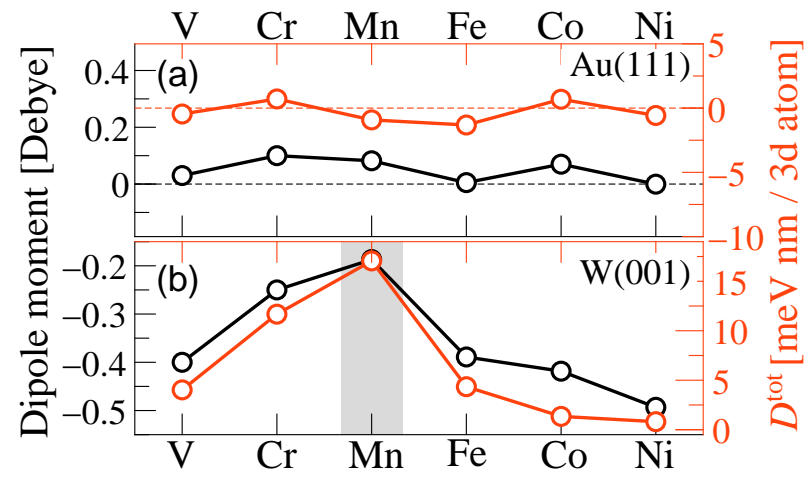

Figure 15: Correlation between Dzyaloshinskii-Moriya interaction (DMI) and the electric surface dipole moment (Dm) for $3 d$ transition metal monolayers on Au (111) (a) and W (001) (b) substrates

mechanism responsible for DMI is intimately related to interfacial charge transfer between the $3 d$ overlayer and the $5 d$ substrate close to the Fermi level. More specifically, by changing the electronic occupation of the $3 d$-orbitals the $3 d-5 d$ hybridization-driven effects such as symmetry of surface wave-function comes into play which is also crucial for the appearance of the DMI and can be easily influenced by changes in surface dipole moment. At first glance, the dipole moment in $3 d / \mathrm{W}(001)$ interfaces, displayed in Fig. 15(b), is much more revealing than $3 d / \mathrm{Au}(111)$ Fig. 15(a). According to this figure, since the $5 d$ states are partially filled the origin of this effect can be easily understood on the basis of the symmetry of $3 d$ orbitals and their energetic positions upon hybridization with $5 d$ orbitals. In the case of $\mathrm{W}(001)$ surface, apart from Mn overlayer, switching from $\mathrm{V}$ to Ni moves up the Fermi level across the $3 d$ bands of transition metal adatoms, in which the Fermi energy is located inside one of the spin subbands and charge transfer occur depending on their filling. As a consequence the surface dipole moment increases the interface symmetry, thereby reducing the DMI energy for $3 d$ elements on both side of $\mathrm{Mn}$ atom. Interestingly, the trend of the electric dipole moment shows an inverse correlation with the DMI. However, in the case of $\mathrm{Mn} / \mathrm{W}(001)$ the $\mathrm{Mn}$ majority $d$ band is nearly filled and the minority $d$ band is nearly empty, resulting in a large interlayer distance [93]. As a result, the dipole moment becomes weaker and the large asymmetry of the $5 d$ surface state wave function remains unaffected, which contributes strongly to DMI in presence of large spin-orbit coupling. In contrast, for the $3 d / \mathrm{Au}(111)$ interfaces, the dipole moments remain small while DMI almost vanishes [Fig. 15(a)]. Obviously, this is a consequence of the weak hybridization between $3 d$ overlayer and the deeper $5 d$-states of Au substrate. More specifically, the changes in the DMI strength and induced dipole moment over the range of the $3 d$ series are only of the order of $\sim 2 \mathrm{meV} \mathrm{nm}$ and $\sim 0.1$ Debye, respectively.

The above results support the physical picture that DMI is not only driven by the spin-orbit coupling of $5 d$ substrate and the inversion symmetry breaking 
but also by the contribution of the intra-atomic exchange field of $3 d$ overlayer which is responsible for the correlated behavior. In this context, these findings are consistent with atomic Hund's rule-type arguments [88] where the maximum of the magnetic moment occurs for half band-filling of $d$ states and, hence, the spin-flip excitation process necessary for DMI is larger in the presence of spinorbit coupling.

\subsection{Controlling DMI}

According to the previous Section, we demonstrated that the magnetic interactions depend sensitively on the band alignment of $3 d$ and $5 d$ spin channels, where a tiny modification of those states at the Fermi level can dramatically impact the induced dipole moment and consequently the DMI. Therefore, controlling the electric dipole moment, using various tunable physical effects as external perturbations, e.g., temperature, strain, electric or magnetic field, doping, electrolyte charging etc., could allow manipulation of the complex spin textures at the atomic scale and, thus, potentially enables predicting and engineering new exotic magnetic phases.

In this respect, we present a new physical process to fine tune the DMI by controlling the electrical dipole moment of $3 d / 5 d$ interfaces via adsorption of electronegative atoms. In order to validate this idea we demonstrate that the sign and strength of the DMI at asymmetric magnetic ultrathin films can be tailored by manipulating the oxygen coverage as a capping layer. In principle, the concept of surface functionalization is more general and not limited to only adatoms whose electronegativity is larger than that of ferromagnet, but can be extended for other electropositive adsorbates. An analogue mechanism based on the concept of surface-charging effect has been successfully applied to tune the magnetic anisotropy energy [94].

Among these adsorbates we have specially chosen the oxygen in this study for the following reasons: (i) from the experimental point of view, the absorption of a very small amount of oxygen is certainly unavoidable during the growth process, and this can happen even under high vacuum conditions, (ii) most first principles calculations on DMI were focused on clean surfaces without oxidants, and (iii) since the oxygen is an electronegative atom, the charge transfer toward oxygen should be significant and, consequently, the interfacial electric dipole moment can be easily tuned by the degree of surface oxidation. In this context, the presence of oxygen at the surface should not be considered as a serious obstacle for growing magnetic nanodevices, but it can prove very useful as a tunable parameter for the functionalization of their magnetic interactions [95, 96, 97]. For instance, the oxidation of CoFeB layer at certain level of oxygen coverage can greatly tune the magnetic anisotropy [98, 99], magnetoresistance properties [100, 101], and spin-orbit torque [102]. Recently, Krupin et al. [103] have also demonstrated a change of Rashba splitting and its sign when oxygen adsorbed on the top of $\operatorname{Gd}(0001)$ surface. Furthermore, recent ab initio study by Yuan et al. [104] demonstrated that manipulating the oxygen concentration on Pt-nanojunction can tune the sign and magnitude of DMI and, consequently, leads to the formation of spin-spirals with different sense of rotations. 
For the sake of clarity, we consider the oxygen adsorption on $\mathrm{Fe} / \mathrm{Ir}(001)$ as a prototype interface of complex magnetic oxides. As a matter of fact, this interface has attracted much attention lately because its strong DMI can promote in a great variety of complex magnetic phases, such as skyrmions or homochiral spin spirals $[105,43,55,66,21,70]$. More specifically, because of the degeneracy of the nearest-neighbor exchange interactions, FM and AFM coupling of the deposited Fe atoms, in clean $\mathrm{Fe} / \mathrm{Ir}(001)$ interface we believe that the electric dipole moment induced by oxygen adsorption is useful to control the competition between Heisenberg exchange interaction, magnetic energy anisotropy, and DMI.

In Figure 16(b), we show the variation of DMI and the electric dipole moment as a function of oxygen coverage. We find a clear chemical tendency when varying the oxygen coverage on $\mathrm{Fe} / \mathrm{Ir}(001)$, in which the magnitude and sign of DMI are strongly correlated with the induced electric dipole moment. This fact actually depends on the balance of the interfacial charge transfer between both Fe-Ir and Fe-O layers, as shown in Fig. 16(a). Indeed, oxygen with O-2p orbital affects the energetic ordering of Fe- $3 d$ spin channels with respect to the Fermi level and, consequently, controls the hybridization between Fe-3d and Ir$5 d$ states and their band lineup. Physically, this correlation is very interesting since the electric dipole moment also depends sensitively on relative energy position of $3 d$ and $5 d$ states around the Fermi energy. The latter demonstrates the close link between the DMI and dipole moment depending on the charge rearrangement between the $3 d$ and $5 d$ spin channels and their energetic position.

The most striking feature in Fig. 16(b) is the sign variation of DMI with increasing the $\mathrm{O}$ coverage beyond $0.25 \mathrm{ML}$. More specifically, the magnetic chirality exhibits an oscillatory behavior between the left and right hand, accompanied by a significant reduction in magnitude of DMI for higher coverages compared to clean surface. These trends are found to be consistent with the energetic shift of the Fermi level $\epsilon_{\mathrm{F}}$ with respect to unperturbed one $\epsilon_{\mathrm{F}}^{0}$ for spin spiral of wave vector $q^{\prime}=0.25 \frac{2 \pi}{a^{\prime}}$ [see Fig. 16(c)]. Note that the $\epsilon_{\mathrm{F}}^{0}$ and $\epsilon_{\mathrm{F}}$ are the Fermi levels obtained without and with spin-orbit coupling, respectively. It is interesting to note that the weak surface dipole moment and DMI at 0.75 ML is traced back to the significant reduction of the electrostatic interactions between the adsorbed oxygen atoms. As a result, surface depolarization occurs because of inversion direction of the dipole moment, indicating that a partial electron charge is transferred from the oxygen overlayer back to Fe atoms. This behavior is clearly visible when inspecting the charge density difference at the neighboring $\mathrm{O}$ (2-3) atoms in Fig. 16(e) compared to 0.25 ML in Fig. 16(d). First principles calculations by Zhang et al. [106] also reported almost similar effect that can be obtained at oxygen rich condition. This mechanism principally drives the induced electric dipole moment and the resulting oscillatory variation of DMI as a function of oxygen coverage. Therefore, our findings demonstrate that the existence of a charge accumulation and depletion at the surface of $3 d / 5 d$ systems is very efficient in controlling the work function [see Fig. 16(b)]. The latter plays an important role to control the induced electric dipole moment and thus the sign and strength of DMI. 

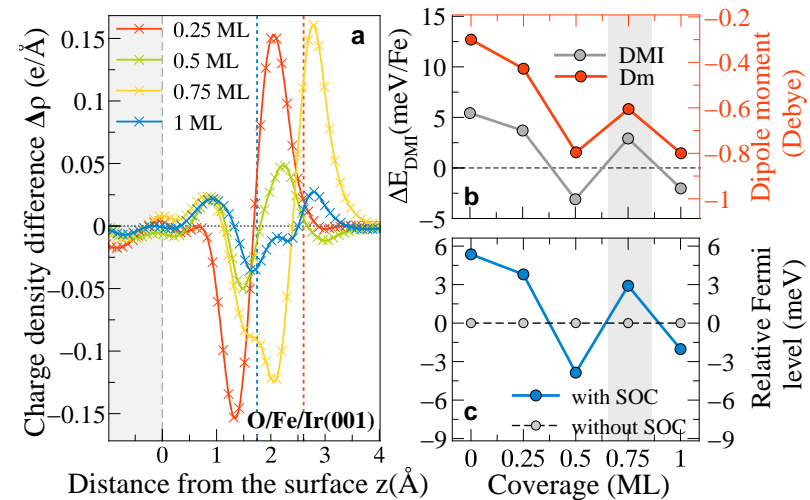

Distance from the surface $\mathrm{z}(\AA)$
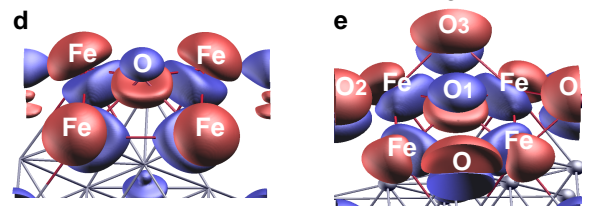

Figure 16: Correlation between Dzyaloshinskii-Moriya interaction (DMI) and the electric surface dipole moment (Dm). (a) Planar averaged charge density difference $\Delta \rho(z)$ for the adsorption of $\mathrm{O}$ on $\mathrm{Fe} / \mathrm{Ir}(001)$ at different coverages. The dashed red $(\mathrm{O})$, blue $(\mathrm{Fe})$, and gray (Ir-surface) lines show the approximate equilibrium position on the fully relaxed surface. $\mathrm{O}$ coverage dependence of the change in the surface dipole moment (Dm), DMI (b) and relative Fermi energy (c). Isosurface plot of the charge density difference $\Delta \rho(\mathbf{r})$ for $0.25(\mathrm{~d})$ and $0.75 \mathrm{ML}(\mathrm{e})$, red and blue surfaces depict the region of charge accumulation and depletion, respectively. The selected surfaces correspond to the charge isodensity of $1.2 \times 10^{-2} e / \AA^{3}$. From [84].

From the above discussion, we conclude that the DMI is a subtle physical mechanism that depends sensitively on the details of the electronic band structure around the Fermi level. This holds particularly true since the DMI involves transitions from the occupied states spin-up to the unoccupied states spin-down and vice versa. As long as the influence of oxygen on the orbital occupation is strong, the positions of these states can be shifted to higher or lower energies depending on the oxygen coverage on top. On the other hand, we emphasize that the energy shift due to the spin-orbit coupling correction also depends on the resulting charge rearrangement between $3 d / 5 d$ orbitals of opposite spin, as discussed in the previous Section. Finally, since controlling the magnetic interactions, in particular the DMI, has been investigated only recently, we anticipate that the prediction and design of complex spin textures via surface functionalization are likely to have major impact in the near-future. 


\section{Spin-Orbit Torques}

\subsection{Overview}

Let us now turn our attention towards the non-equilibrium properties of transition metal interfaces. In magnetic bilayers composed of a (metallic or insulating) magnet deposited on top of a heavy metal (W, Pt, $\mathrm{Bi}_{2} \mathrm{Se}_{3}, \mathrm{WTe}_{2}$ etc.), the flowing charges experience spin-charge conversion mechanisms that produce a non-equilibrium spin accumulation at the interface with the ferromagnet, such that an overall torque is exerted on the magnetization. This effect, called spinorbit torque, has been initially observed in transition metal bilayers $[107,108,8$, $9,109]$ and bulk non-centrosymmetric magnets $[110,111,112]$, and recently extended to a wide variety of transition metal bilayers [113, 109, 67, 114, 115, 116], oxide interfaces [117, 102, 118], thick multilayers [119, 120], magnetic insulators deposited on heavy metals [121, 122, 123], as well as ferromagnets deposited on topological insulators [124, 125, 126, 127], and Weyl semimetals [128].

Overall, the experiments confirm that the torque is of the form $\boldsymbol{\tau}=\tau_{\mathrm{DL}} \mathbf{m} \times$ $[(\mathbf{z} \times \mathbf{u}) \times \mathbf{m}]+\tau_{\mathrm{FL}} \mathbf{m} \times(\mathbf{z} \times \mathbf{u})$, where $\mathbf{z}$ is the normal to the interface and $\mathbf{u}$ is the direction of injection of the flowing charges. The first term $\sim \tau_{\mathrm{DL}}$ is the damping-like torque while the second term $\sim \tau_{\mathrm{FL}}$ is called the fieldlike torque. Both damping-like and field-like torques are present in most of the systems involving heavy metals. Uncovering the physical mechanisms that sustain the spin-orbit torque is quite challenging, as many effects are present in such ultrathin systems. In a nutshell, two main effects have been pointed out originally: the inverse spin-galvanic effect, localized at the interface between the heavy metal (or the oxide) and the ferromagnet, and the spin Hall effect present in the bulk of the heavy metal, as illustrated on Fig. 17. As explained in more details below, the former generates a field-like torque $[129,130,131] \sim$ $\mathbf{m} \times(\mathbf{z} \times \mathbf{u})$, while the spin Hall effect generates a damping-like torque [108, 132] $\sim \mathbf{m} \times[(\mathbf{z} \times \mathbf{u}) \times \mathbf{m}]$. However, only a few systems, such as the $\mathrm{Ti} / \mathrm{NiFe} / \mathrm{AlOx}$ trilayers from Emori et al. [118], can be reasonably well explained by these two scenarii. In most cases, both torques are observed and their materials dependence raises many questions concerning the physical mechanism at their origin. More recently, two additional mechanisms have been proposed: the magnetoelectric effect and the spin swapping effect. The former is a correction to the inverse spin galvanic effect when inversion symmetry breaking, spin-orbit coupling and magnetic exchange are present on the same orbitals, and generates a damping-like torque $[133,134,135]$. The latter is a correction to the spin Hall effect and only survives in ultrathin multilayers as thin as the mean free path [136]. It creates a field-like torque.

Our objective is to present these various mechanisms, and discuss their physical origin and their impact on the spin-orbit torque. We first address the physics of spin Hall and spin swapping in Section 4.2, and discuss the physics of inverse spin galvanic effect and magnetoelectric effect in Section 4.3. 


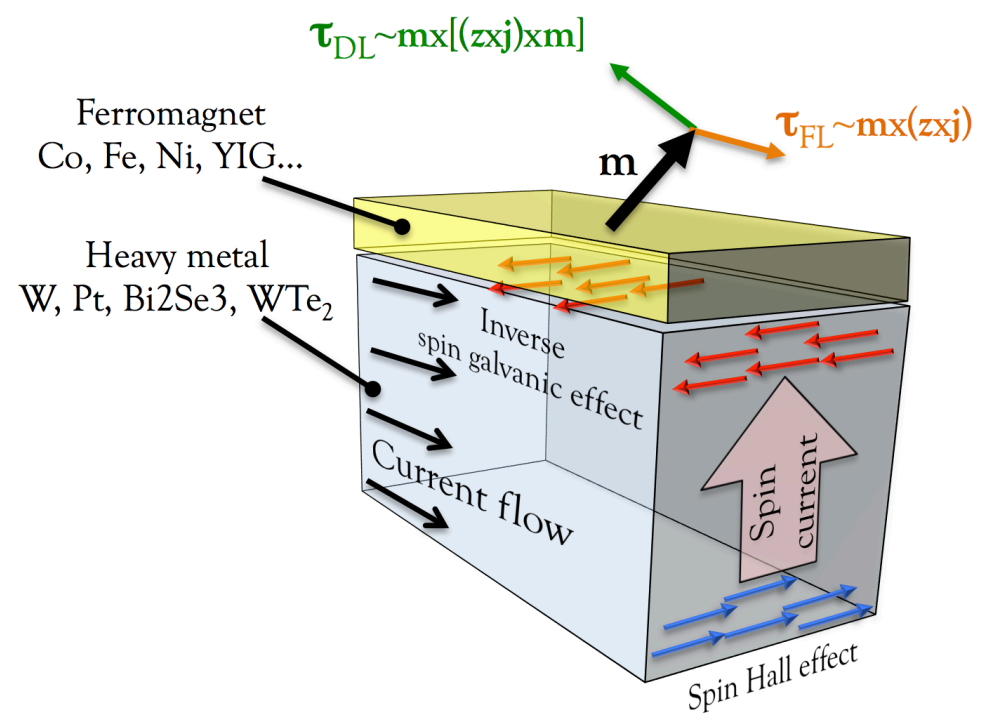

Figure 17: (Color online) In magnetic bilayers involving a ferromagnet deposited on a heavy metal, two main spin-charge conversion mechanisms occur: the inverse spin galvanic effect (also called the Rashba or Rashba-Edelstein effect), and the spin Hall effect. While the inverse spin galvanic effect directly generates a non-equilibrium spin density, $\delta \mathbf{S} \sim \mathbf{z} \times \mathbf{u}$, at the interface with the ferromagnet, the spin Hall effect induces a spin current in the bulk of the heavy metal. This spin current creates a non-equilibrium spin accumulation that diffuses into the ferromagnet. The two mechanisms give rise, at the lowest order, to a field-like (orange arrow) and damping-like torque (green arrow), respectively.

\subsection{Spin Hall and swapping effects}

Spin Hall effect is the conversion of a unpolarized charge flow $\mathbf{j}_{e}$ into a chargeless spin current $\mathcal{J}_{s}$. In the bulk, it reads $e \mathcal{J}_{s}=\theta_{\mathrm{sh}} \boldsymbol{\sigma} \otimes \boldsymbol{\sigma} \times \mathbf{j}_{e}$, where $\boldsymbol{\sigma}$ is the polarization of the spin current. We remind that the spin current $\mathcal{J}_{s}$ is a $3 \times 3$ tensor in spin and real space. $\theta_{\mathrm{sh}}$ is called the spin Hall angle and quantifies the charge-to-spin conversion efficiency. This effect was predicted originally by D'yakonov and Perel $[137,138]$ and revived about twenty years ago by Hirsch [139] and Zhang [140]. It has initially been observed optically in semiconductors $[141,142]$ and measured electrically in transition metals [143, 144]. Since then, spin Hall effect has drawn a massive amount of attention [145, 146, 147, 148, 149] as it offers a convenient way to generate pure spin currents, enabling non-local spin logic [150] as well as promoting spin-orbit torques [8, 108, 9].

Computing non-equilibrium properties of non-collinear magnetic systems in the presence of spin-orbit coupling is in itself a significant challenge since neither the linear momentum nor the spin angular momentum are good quantum numbers in this case. Different approaches have been proposed to tackle this problem. The "simplest" microscopic multi-orbital approach is probably the one adopted by Tanaka et al. [151] and Kontani et al. [152, 153] for noble metals. 
The authors use a tight-binding model accounting for a minimum set of orbitals (e.g. $5 s, 5 p$ and $4 d$ orbitals for $4 d$ noble metals) to calculate the bulk intrinsic spin Hall effect using Kubo formula. This model allows for a rather transparent analysis of the origin of the orbital and spin Hall effects. Computation of intrinsic spin Hall effect based on the Berry phase formula and using ab initio methods has been achieved by Yao et al. $[154,155]$ and Guo et al. $[156,157]$. These studies have been recently extended to account for both extrinsic and intrinsic effects $[158,159,160,161]$ (see also Ref. [162]). In these works, the band structure was obtained through fully relativistic Korringa-Kohn-Rostoker method (see for instance Ref. [163]) and the transport properties were evaluated either through Boltzmann equation [158, 159] or Kubo-Streda formula $[160,161]$. While these different works have shed light on extrinsic and intrinsic spin Hall effects in metals and semiconductors, they focus on bulk materials and do not address the torque arising from spin Hall effect.

We emphasize straightaway that the spin Hall effect is the time-reversal counterpart of the anomalous Hall effect [5]. Therefore, both effects encompass the same classes of mechanisms. In the theoretical investigation of spin Hall effect, two classes of mechanisms have been identified. In the first case, the separation between opposite spins occurs upon scattering against spin-orbit coupled impurities. This class of mechanisms is called extrinsic, as scattering events against impurities govern the spin Hall effect. In the second case, the anomalous velocity arises from the spin-orbit coupling present in the band structure itself. This class of mechanisms is called intrinsic, as the spin-charge conversion process does not require scattering to occur. Distinguishing between these different classes of effects is important from an experimental standpoint, as one might pertinently wonder :" is it more favorable to increase or decrease the amount of impurities in order to obtain large spin-charge conversion efficiency?".

\subsubsection{Impurity scattering driven spin Hall effect}

In the limit of short range (delta-like) impurity potential, the extrinsic spin Hall effect is composed of two mechanisms: the Mott scattering [illustrated on Fig. 18(a)], also called skew scattering, and the side jump. The former was initially proposed by Smit in the context of anomalous Hall effect [164] and by D'yakonov in his pioneering work [138], while the latter was put forward by Berger [165], also in the context of anomalous Hall effect. To see explicitly how spin transport is affected by spin-orbit scattering, let us first consider the Hamiltonian of a spin-orbit coupled impurity (see for instance Ref. [166]). In real space, it reads

$$
\hat{H}^{\mathrm{imp}}=\sum_{i} V_{\mathrm{imp}}\left(\mathbf{r}-\mathbf{r}_{i}\right)+\left(\lambda_{\mathrm{so}} / \hbar\right) \hat{\boldsymbol{\sigma}} \cdot\left[\nabla V_{\mathrm{imp}}\left(\mathbf{r}-\mathbf{r}_{i}\right) \times \hat{\mathbf{p}}\right],
$$

where $V_{\mathrm{imp}}$ is the spin-independent impurity potential, and $\lambda_{\mathrm{so}}$ is the spinorbit coupling parameter of the impurity. In the reciprocal space, this impurity potential becomes

$$
\hat{H}_{\mathbf{k} \mathbf{k}^{\prime}}^{\mathrm{imp}}=\sum_{i} V_{\mathbf{k k}^{\prime}}^{\mathrm{imp}} e^{-i\left(\mathbf{k}-\mathbf{k}^{\prime}\right) \cdot\left(\mathbf{r}-\mathbf{r}_{i}\right)}\left[1+i \lambda_{\mathrm{so}} \hat{\boldsymbol{\sigma}} \cdot\left(\mathbf{k}^{\prime} \times \mathbf{k}\right)\right] .
$$


It is clear that the spin-orbit coupled part of the impurity potential acts like a magnetic field $\mathbf{B}_{\text {so }} \propto \mathbf{k}^{\prime} \times \mathbf{k}$ on the incoming electron spin $\hat{\boldsymbol{\sigma}}$, where $\mathbf{k}$ is the momentum of the incoming electron and $\mathbf{k}^{\prime}$ is the momentum of the outgoing electron. Therefore, in the case of an unpolarized charge current, this magnetic field defines a local quantization axis such that electrons with spins parallel and antiparallel to $\mathbf{B}_{\text {so }}$ experience a different $k$-dependent scattering rate, as illustrated on Fig. 18(a): electrons with a spin momentum pointing (anti)parallel to $\mathbf{k}^{\prime} \times \mathbf{k}$ have the tendency to scatter towards the left (right).

(a) Mott scattering

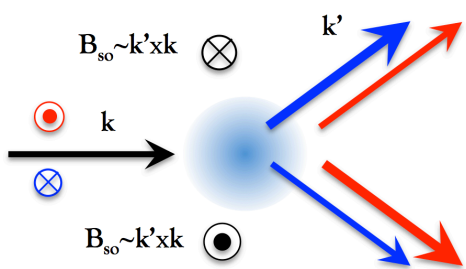

(b) Spin Swapping

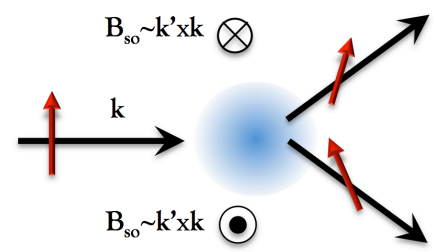

Figure 18: (Color online) (a) Sketch of Mott scattering by a spin-orbit coupled impurity. Electrons with an out-of-plane spin polarization pointing up have a larger probability to scatter towards the right while electrons with an out-of-plane spin polarization pointing down have a larger probability to scatter towards the left. (b) Sketch of spin swapping mediated by a spin-orbit coupled impurity. When the spin of the carrier lies in the scattering plane $\left(\mathbf{k}, \mathbf{k}^{\prime}\right)$, it experiences a magnetic field $\mathbf{B}_{\text {so }} \propto \mathbf{k}^{\prime} \times \mathbf{k}$ that depends on the direction towards which the spin is scattered. This induces a scattering-induced precession giving rise to the spin swapping. From [167].

In addition, the perturbed wave function in the presence of impurities reads

$$
\Psi_{\mathbf{k}}^{s} \sim e^{i \mathbf{k} \cdot \mathbf{r}}+\sum_{i} \sum_{\mathbf{k}^{\prime}} \frac{V_{\mathbf{k k}^{\prime}}^{\mathrm{imp}}}{\epsilon_{\mathbf{k}}^{s}-\epsilon_{\mathbf{k}^{\prime}}^{s}+i 0^{+}} e^{-i\left(\mathbf{k}-\mathbf{k}^{\prime}\right) \cdot\left(\mathbf{r}-\mathbf{r}_{i}\right)} e^{i \mathbf{k}^{\prime} \cdot \mathbf{r}} e^{i s \lambda_{\mathrm{so}} \mathbf{k}^{\prime} \cdot(\mathbf{n} \times \mathbf{k})},
$$

where $s$ is the spin projection perpendicular to the scattering plane, defined by the unit vector $\mathbf{n}$. One can see that the scattering wave function acquires a spin-dependent phase $\sim s \lambda_{\text {so }} \mathbf{n} \cdot\left(\mathbf{k} \times \mathbf{k}^{\prime}\right)$ due to the local spin-orbit field. This phase can be rewritten in term of a spin-dependent spatial displacement $\boldsymbol{\delta}=s\left(\lambda_{\mathrm{so}} / \hbar\right)(\mathbf{n} \times \mathbf{p})$ [166], which is referred to as side-jump scattering. The skew scattering produces a spin Hall conductivity $\sigma_{\mathrm{SH}}^{\text {skew }} \sim \sigma_{x x}$, namely the charge-to-spin conversion efficiency is independent on the scattering strength. In contrast, the side-jump modifies directly the velocity operator of the electron and therefore it gives a spin Hall conductivity $\sigma_{\mathrm{SH}}^{\mathrm{s}-\mathrm{j}} \sim O\left(\sigma_{x x}\right)$, such that the spin Hall angle is inversely proportional to the scattering time: the stronger the scattering the larger the charge-to-spin conversion (see e.g., Ref. [145]).

These extrinsic contributions have been recently investigated using ab initio calculations. It was shown that even light impurities (such as $\mathrm{Li}, \mathrm{C}$ and $\mathrm{N}$ ) embedded in noble metals (such as $\mathrm{Au}$ or $\mathrm{Cu}$ ) could lead to large extrinsic 

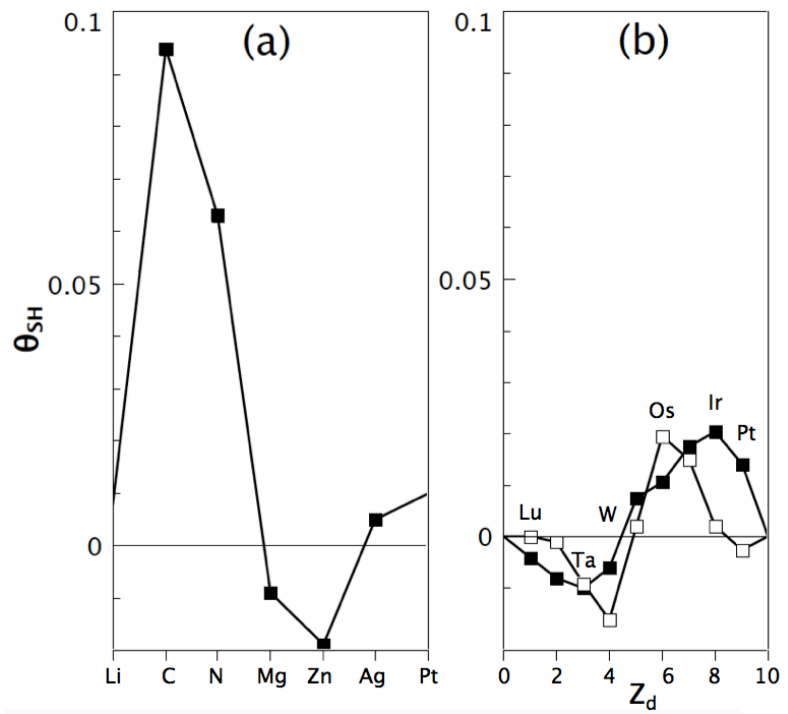

Figure 19: (Color online) (a) Extrinsic spin Hall effect induced by substitutional impurities in $\mathrm{Au}$, computed by first principles calculations [159]. (b) Extrinsic spin Hall angle calculated using virtual bound state theory [168]. The filled symbols refer to skew scattering, while the open symbols refer to side-jump scattering. In this model, the metal is $\mathrm{Cu}$ and the $5 d$ impurity possess $Z_{d}$ electrons.

spin Hall contributions (up to $10 \%$ in C-doped $\mathrm{Au}$ ) [158, 159], as illustrated on Fig 19(a). The long-standing issue of the role of side-jump versus intrinsic contributions has been addressed by Lowitzer et al. [161], who demonstrated that side jump is general weak in alloys (except for the noticeable case of $\mathrm{Au}-$ doped $\mathrm{Ag}$ ). Finally, the authors discovered that the sign of the spin Hall effect may also change as a function of the composition of the alloy.

Fert and Levy $[168,169]$ and Gu et al. [170] recently suggested that resonant scattering on $d$ or $p$ impurities could lead to large spin Hall angle, as illustrated on Fig 19(b). The spin Hall effect resulting from the scattering off the impurity spin-orbit coupled states close to the Fermi energy (such as $j=5 / 2$ and $j=3 / 2$ in the case of Ir-doped $\mathrm{Cu}$, or $j=3 / 2$ and $j=1 / 2$ in the case of Bi-doped $\mathrm{Cu}$ ) depends on the relative phase shifts acquired during scattering. Such phase shifts depend on the orbital filling of the impurities following Friedel's law. This results in a different sign of the Hall angle when considering impurities with less than half-filled shells or more than half-filled shells [171]. Resonant scattering on magnetic impurities has also been investigated by Guo et al. [172] who suggested that orbital-dependent Kondo effect could lead to a large enhancement of the skew scattering and applied it to Fe-doped $\mathrm{Au}$ compounds [173]. Resonant scattering on $\mathrm{Ce}$ and $\mathrm{Yb}$ magnetic impurities has been addressed by Tanaka and Kontani [174]. Evidence of large Hall angles from resonant skew scattering was reported in Pt- [173, 170] and W-doped Au [175], as well as Ir- [176] and Bi-doped $\mathrm{Cu}$ [177]. 


\subsubsection{Intrinsic spin Hall effect in transition metals}

The prediction of the intrinsic spin Hall effect by Murakami et al. [178] and Sinova et al. [179] opened thrilling perspectives. The main idea is that the spinorbit coupling present in the band structure gives a contribution to the velocity operator that depends on the spin projection, and can be directly related to the Berry curvature of the materials. Since intrinsic spin Hall effect arises from the distortion of the wave function in the presence of an electric field, it does not require the subtle treatment of impurity scattering beyond the relaxation time approximation and can be readily implemented in density functional theory. The spin Hall conductivity reads

$$
\sigma_{x y}=\frac{e^{2}}{\Omega} \sum_{n, m, \mathbf{k}} \frac{2 \Im\left[\left\langle n\left|\hat{v}_{y}\right| m\right\rangle\left\langle n\left|\hat{v}_{x}\right| m\right\rangle\right]}{\left(\epsilon_{n, \mathbf{k}}-\epsilon_{m, \mathbf{k}}\right)^{2}}\left(f\left(\epsilon_{n, \mathbf{k}}\right)-f\left(\epsilon_{m, \mathbf{k}}\right)\right) .
$$

This term involves electric-field driven interband transitions and does not depend on the amount of disorder (at least in the limit of weak disorder). Guo et al. [157] and Kontani [152] predicted large intrinsic spin Hall effect in Pt and $\mathrm{Sr}_{2} \mathrm{MO}_{4}$ semiconductors, respectively. These studies were shortly followed by in-depth analysis of the origin of intrinsic spin Hall effect in metals, showing that the Berry curvature of the bands promotes a large orbital Hall effect, which induces spin Hall effect that is proportional to the spin-orbit coupling [153, 151]. Interestingly, the intrinsic orbital Hall effect is associated to "hot spots" in the band structure occurring at anti crossing points, which is a direct consequence of the denominator $\sim\left(\epsilon_{n, \mathbf{k}}-\epsilon_{m, \mathbf{k}}\right)^{2}$ obtained from the first order perturbation theory given above. Note that intrinsic spin Hall effect in crystalline materials displays anisotropy [180].
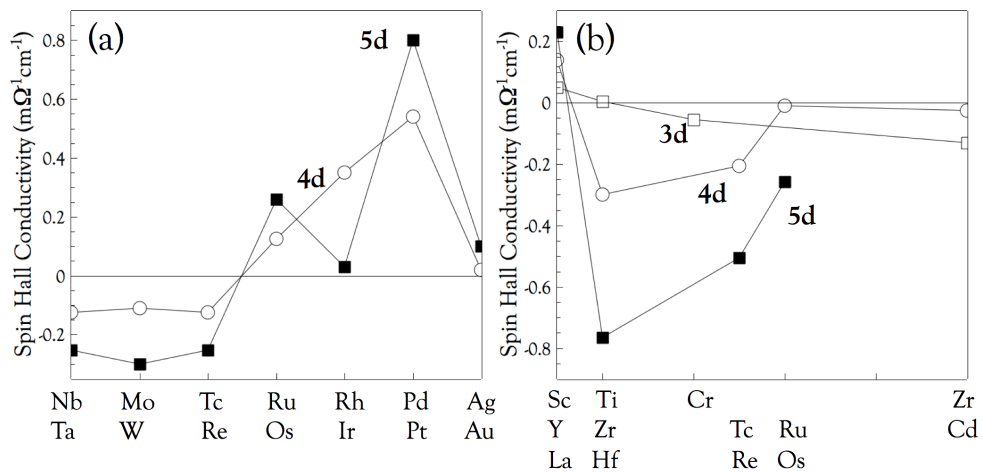

Figure 20: (Color online) Intrinsic spin Hall effect computed using two different methods: (a) 9-orbital tight-binding model [151], and (b) using the full-potential linearized augmentedplane-wave code FLEUR [180].

An instructive aspect of the intrinsic spin Hall effect is the fact that the sign of the spin Hall angle is directly related to the band filling. Tanaka et al. [151] 
observed that the intrinsic spin Hall effect is positive in noble metals with more than half filled $d$ band (Pt, $\mathrm{Pd}, \mathrm{Ir}, \mathrm{Au}, \mathrm{Ag}$, and $\mathrm{Rh}$ ), while the spin Hall effect becomes negative in heavy metals with $d$ bands less than half filled $(\mathrm{Ta}, \mathrm{Nb}, \mathrm{W}$ and Mo) [see Fig. 20(a)]. Indeed, Hund's third rule states that in a spin-orbit coupled system where the total angular momentum $\mathbf{J}=\mathbf{L}+\mathbf{S}$ is a good quantum number, $J=|L-S|$ if the shell is less than half-filled and $J=|L+S|$ is the shell is more than half-filled. Therefore, while the orbital Hall current is set by the crystalline environment, the spin Hall current is opposite to the orbital Hall current in materials with less than half-filled shells and along the orbital Hall current in materials with more than half-filled shells. A similar trend has been obtained by Freimuth et al. [180] [see Fig. 20(b)]. Notice though that the two calculations reported on Fig. 20 show some quantitative differences, that could be attributed to the different computational methods. This trend is consistent with experimental observations [181]. Intrinsic spin Hall effect has also been recently investigated in transition metal antiferromagnets $\mathrm{XMn}(\mathrm{X}=\mathrm{Pt}, \mathrm{Ir}, \mathrm{Pd}$, Fe) [182]. The authors found sizable values of the spin Hall effect, together with large crystalline anisotropy.

\subsubsection{Interfacial spin Hall effect}

The extrinsic and intrinsic contributions we discussed in the previous Sections have been computed in the bulk of heavy metals. However, the vast majority of the devices on which spin Hall effect is measured consists of thin heavy metals in contact with ferromagnets. As discussed in Section 2, such interfaces involve strong orbital hybridization such that the orbital character of the wave functions close to the interface may be very different from the one of the bulk Bloch states, resulting in enhanced orbital angular momentum (see Fig. 5) and Rashba-type spin-orbit coupling (see Fig. 6). As a result, one can reasonably expect the charge-to-spin conversion process to be dramatically modified at the interface. This effect has been uncovered very recently by Wang et al. [183]. Using tight-binding linear muffin-tin orbitals, the authors investigate the spin Hall effect occurring along the interface of a $\mathrm{Pt} / \mathrm{NiFe}$ system and found a very large enhancement of the spin Hall angle $\left(\theta_{\mathrm{sh}} \approx 0.87\right.$ at the interface, compared to 0.03 in bulk $\mathrm{Pt}$ ). This observation opens inspiring directions for a better understanding of spin-charge conversion processes at transition metal interfaces.

\subsubsection{Spin swapping}

Another effect occurs together with the extrinsic spin Hall effect. Let us consider again the spin-orbit coupled Hamiltonian given in Eq. 15. We already stressed out that the spin-orbit coupling acts like a $k$-dependent Zeeman field, normal to the scattering plane. In the case of unpolarized electrons, this Zeeman field is responsible for skew scattering [Fig. 18(a)]. Let us now consider an incoming spin-polarized current, whose spin polarization lies in the scattering plane. In this case, the incoming spin precesses around the effective magnetic field $\mathbf{B}_{\mathrm{so}}$. Since this magnetic field only exists upon scattering from $\mathbf{k}$ to $\mathbf{k}^{\prime}$, the spin polarization of the outgoing current is re-oriented [184]. Lifshits and Dyakonov tagged this effect "spin swapping" as the spin polarization and flow 
direction of the incoming spin current are swapped during this process: an incoming spin current $\mathcal{J}_{s, j}^{i}$, flowing along $\mathbf{e}_{j}$ and polarized along $\mathbf{e}_{i}$, gives rise to a spin current $\mathcal{J}_{s, i}^{j}$, flowing along $\mathbf{e}_{i}$ and polarized along $\mathbf{e}_{j}$, when $i \neq j$, as illustrated on Fig. 18(b). Consistently, a spin current $\mathcal{J}_{s, x}^{x}$ produces two spin currents $\mathcal{J}_{s, y}^{y}$ and $\mathcal{J}_{s, z}^{z}$.

Spin swapping has been derived by several authors within the diffusive limit [185, 186, 187, 188]. For instance, Shchelushkin and Brataas obtained the expression of the charge and spin currents [185, 189]

$$
\begin{aligned}
\mathbf{j}_{e} / \sigma_{0} & =-\nabla \mu_{c}+\frac{\xi_{\text {so }}}{\lambda k_{\mathrm{F}}} \nabla \times \boldsymbol{\mu}_{s}, \\
e \mathcal{J}_{s, i} / \sigma_{0} & =-\nabla_{i} \boldsymbol{\mu}_{s}-\frac{\xi_{\mathrm{so}}}{\lambda k_{\mathrm{F}}} \mathbf{e}_{i} \times \nabla \mu_{c}+\frac{2 \xi_{\mathrm{so}}}{3} \nabla \times\left(\mathbf{e}_{i} \times \boldsymbol{\mu}_{s}\right) .
\end{aligned}
$$

Here, $\mathcal{J}_{s, i}$ is the spin current density flowing along the direction $\mathbf{e}_{i}, \mu_{c}$ and $\boldsymbol{\mu}_{s}$ are the spin-independent and spin-dependent chemical potentials, $\sigma_{0}$ is Drude conductivity and $\xi_{\mathrm{so}}=\lambda_{\mathrm{so}} k_{\mathrm{F}}^{2}$ is the unitless spin-orbit parameter. The metallic system is described in terms of its free electron Fermi wave vector $k_{\mathrm{F}}$ and its mean free path $\lambda$. The first terms in Eqs. (18) and (19) are the diffusion terms, the second terms $\left(\propto \xi_{\mathrm{so}} / \lambda k_{\mathrm{F}}\right)$ are the side jump contributions producing spin Hall effect [Eq. (19)] and inverse spin Hall effect [Eq. (18)]. The third term in Eq. (19) is the spin swapping effect. Note that since these equations are derived within the first Born approximation (i.e. up to the second order in impurity potential $\left|V_{\mathbf{k k}^{\prime}}^{\mathrm{imp}}\right|^{2}$ only), skew scattering is neglected.

In order to visualize what spin swapping does, let us consider a tight-binding model that represents a magnetic electrode connected to a non-magnetic layer that possesses spin-orbit coupling. The idea is to inject a spin-polarized current from a ferromagnet into a non-magnetic, spin-orbit coupled channel and observe the spin accumulation along the edges of the channel. The model is a simple spin-polarized single-band, where impurity-driven spin-orbit coupling is modeled as a spin-dependent second-nearest neighbor hopping [167]. The system is a two-dimensional square lattice connected laterally to external leads. The full Hamiltonian of the central system reads

$$
\begin{aligned}
\hat{\mathcal{H}}= & \sum_{i, j}\left(\epsilon_{i j} \hat{c}_{i, j}^{\dagger} \hat{c}_{i, j}+\Delta_{i j} \hat{c}_{i, j}^{\dagger} \hat{\sigma} \cdot \mathbf{m} \hat{c}_{i, j}-t_{\mathrm{N}}\left(\hat{c}_{i+1, j}^{\dagger} \hat{c}_{i, j}+\hat{c}_{i, j+1}^{\dagger} \hat{c}_{i, j}\right)+h . c .\right) \\
& -\sum_{i, j}\left(t_{i-1, j-1}^{i, j} \hat{c}_{i, j}^{\dagger} \hat{\sigma}_{z} \hat{c}_{i-1, j-1}+t_{i-1, j}^{i, j-1} \hat{c}_{i, j-1}^{\dagger} \hat{\sigma}_{z} \hat{c}_{i-1, j}+\text { h.c. }\right) .
\end{aligned}
$$

The operator $\hat{c}_{i, j}^{\dagger}=\left(c_{i, j}^{\uparrow}, c_{i, j}^{\downarrow}\right)^{\dagger}$ creates a particle at position $(i, j)$. Here the first line at the right-hand side of Eq. (20) contains the energy on site $(i, j)\left(\sim \epsilon_{i j}\right)$, the magnetic exchange $\left(\sim \Delta_{i j}\right)$ and the nearest neighbor hopping $\left(\sim t_{\mathrm{N}}\right)$. The site energy is defined $\epsilon_{i j}=\epsilon_{0}+\gamma_{i j}, \epsilon_{0}$ being the unperturbed onsite energy and $\gamma_{i j} \in[-\Gamma / 2, \Gamma / 2]$ a random potential of strength $\Gamma$ that introduces disorder in the system. The second line is the next-nearest neighbor hopping that account for the disorder-driven spin-orbit coupled scattering. The nextnearest neighbor hopping parameter reads $t_{i^{\prime}, j^{\prime}}^{i, j}=i t_{\mathrm{N}} \alpha\left(\epsilon_{i, j^{\prime}}-\epsilon_{i^{\prime}, j}\right)$, where $\alpha$ is 


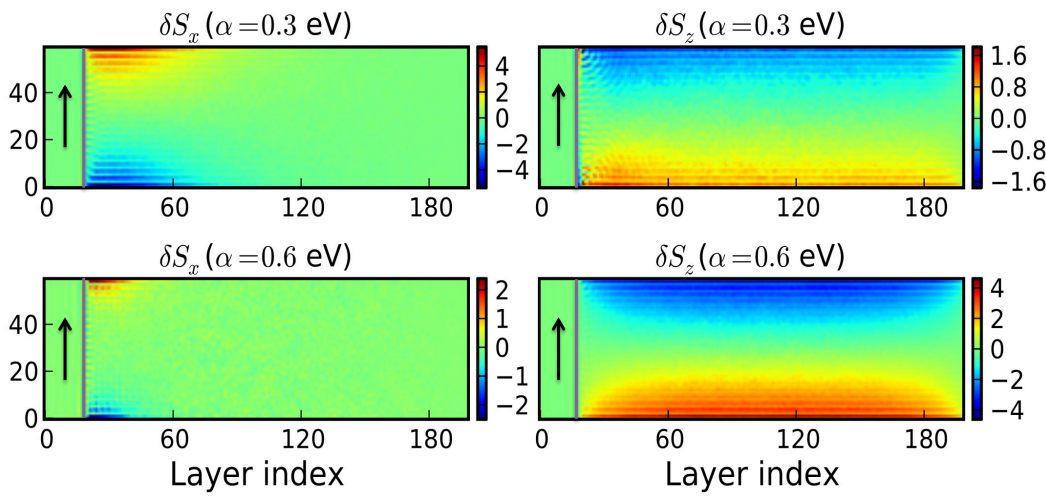

Figure 21: (Color online) Two dimensional mapping of the (a,c) $x$ - and (b,d) $z$-components of the spin density (in unit of $10^{-3} \hbar / 2$ ) demonstrating the crossover between extrinsic spin swapping and extrinsic spin Hall effect, for $(\mathrm{a}, \mathrm{b}) \alpha=0.3$ and $(\mathrm{c}, \mathrm{d}) \alpha=0.6$. The disorder strength is $\Gamma=1 \mathrm{eV}$. The first 20 layers compose the ferromagnetic edge whose magnetization direction is indicated by the black arrow. From Ref. [167]

the dimensionless spin-orbit coupling strength. This approach models extrinsic spin Hall effect and spin swapping on equal footing [167]. We emphasize that the calculations need to be performed over a very large $\left(\sim 10^{5}\right)$ number of disorder configurations to enable the buildup of the spin accumulation.

Figure 21 represents the ferromagnetic layer (green region with the black arrow) adjacent to the normal metal in which extrinsic spin-charge conversion processes occur. In this work, we apply a small potential gradient across the system along $x$, the magnetization of the ferromagnet is set transverse to the flowing current direction, i.e. along $y$, and we compute the two dimensional map of the spin density components, $\delta S_{x}$ and $\delta S_{z}$. The former is due to spin swapping, i.e. from the conversion of the injected spin current $\mathcal{J}_{s, x}^{y}$ (flowing along $\mathrm{x}$ and polarized along $y$ ) into $\mathcal{J}_{s, y}^{x}$ (flowing along $y$ and polarized along $x$ ). The latter is due to the spin Hall effect that converts the current flowing along $x$ into a spin current $\mathcal{J}_{s, y}^{z}$ (polarized along $z$ ). The spin swapping mechanism results in a spin accumulation $\delta S_{x}$, that only survives close to the interface with the ferromagnet. Away from the interface, at a distance corresponding to the spin relaxation length, $\delta S_{x}$ vanishes and $\delta S_{z}$ builds up, due to spin Hall effect [167]. As discussed below, this effect leads to unexpected torques in ultrathin bilayers.

\subsubsection{Spin Hall and swapping torques}

Let us now consider a bilayer composed of a ferromagnet on top of a heavy metal. The spin Hall and spin swapping effects produce a spin accumulation at the interface with the ferromagnet, which results in two main effects: a spin torque on the magnetization $[108,132]$ and an associated magnetoresistance 
effect [190]. The diffusive model given above can be solved to obtain the torque exerted on the adjacent layer. Using the spin mixing conductance [191] to represent the spin absorption at the magnetic interface, we obtain [136]

$$
\begin{aligned}
\boldsymbol{\tau}_{\mathrm{sh}}= & \frac{\hbar}{2 e} \frac{\tilde{\theta}_{\mathrm{sh}}}{D_{\theta}} \eta_{0}\left[-\left(\tilde{g}_{r}^{\uparrow \downarrow}+\left|\tilde{g}^{\uparrow \downarrow}\right|^{2}\right) \mathbf{m} \times\left[\left(\mathbf{z} \times \mathbf{j}_{e}\right) \times \mathbf{m}\right]+\tilde{g}_{i}^{\uparrow \downarrow} \mathbf{m} \times\left(\mathbf{z} \times \mathbf{j}_{e}\right)\right](21) \\
\boldsymbol{\tau}_{\mathrm{sw}}= & \frac{\hbar}{2 e} \theta_{\mathrm{sw}} \frac{\tilde{\theta}_{\mathrm{sh}}}{D_{\theta}}\left[\left(\left|\tilde{g}^{\uparrow \downarrow}\right|^{2}-\eta \tilde{g}_{r}^{\uparrow \downarrow}\right) m_{z} \mathbf{m} \times \mathbf{j}_{e}+\eta \tilde{g}_{i}^{\uparrow \downarrow} m_{z} \mathbf{m} \times\left(\mathbf{j}_{e} \times \mathbf{m}\right)\right], \\
D_{\theta} \approx & \eta_{0}\left[\left(\tilde{g}_{i}^{\uparrow \downarrow}\right)^{2}+\left(1+\tilde{g}_{r}^{\uparrow \downarrow}\right)\left(1+\tilde{g}_{r}^{\uparrow \downarrow}+\theta_{\mathrm{sw}}\right)\right] \\
& -\theta_{\mathrm{sw}} m_{z}^{2}\left[\left(\tilde{g}_{i}^{\uparrow \downarrow}\right)^{2}+\left(\tilde{g}_{r}^{\uparrow \downarrow}-\eta\right)\left(1+\tilde{g}_{r}^{\uparrow \downarrow}\right)\right]
\end{aligned}
$$

Here $\sigma_{\mathrm{F}, \mathrm{N}}$ and $\lambda_{\mathrm{sf}}^{\mathrm{F}, \mathrm{N}}$ are the conductivity and spin diffusion length of the ferromagnetic (normal metal) layer, and $\mathbf{j} e$ is the charge current density flowing in the normal metal. In order to keep the notation compact, we defined the effective spin Hall angle $\tilde{\theta}_{\mathrm{sh}}=\theta_{\mathrm{sh}}\left(1-\cosh ^{-1} d_{\mathrm{N}} / \lambda_{\mathrm{sf}}^{\mathrm{N}}\right)$ and normalized mixing conductances $\tilde{g}_{j}^{\uparrow \downarrow}=4 \tilde{\lambda}_{\mathrm{sf}}^{\mathrm{N}} g_{j}^{\uparrow \downarrow} / \sigma_{\mathrm{N}}$, where $\tilde{\lambda}_{\mathrm{sf}}^{\mathrm{N}}=\lambda_{\mathrm{sf}}^{\mathrm{N}} / \tanh \left(d_{\mathrm{N}} / \lambda_{\mathrm{sf}}^{\mathrm{N}}\right)$ is the effective spin diffusion length of the normal metal. Finally, $\eta=\frac{4\left(1-\gamma^{2}\right) g \tilde{\lambda}_{\mathrm{sh}}^{\mathrm{N}} / \sigma_{\mathrm{N}}}{1+4\left(1-\gamma^{2}\right) g \bar{\lambda}_{\mathrm{sf}}^{\mathrm{F}} / \sigma_{\mathrm{F}}}$, and $\eta_{0}=1+\eta+\theta_{\mathrm{sw}}$, where $g$ is the interfacial conductance. The spin Hall effect produces both a damping-like torque and a field-like torque. While the former is related to the interfacial absorption of the spin current via the real part of the mixing conductance $\left(\sim g_{r}^{\uparrow \downarrow}\right)$, the latter is associated with spin precession at the interface via the imaginary part of the mixing conductance $\left(\sim g_{i}^{\uparrow \downarrow}\right)$. In general, $g_{i}^{\uparrow \downarrow} \ll g_{r}^{\uparrow \downarrow}[192]$ and the spin Hall effect produces mainly a damping-like torque in the diffusive regime. Within this limit, the spin swapping torque emerges at the second order in spin-orbit coupling $\left(\sim \theta_{\mathrm{sw}} \theta_{\mathrm{sh}}\right)$, and produces also both torques, the dominant one being the damping-like component. As we shall see below, the nature of the torque actually dramatically depends on the transport regime considered.

Because of spin transfer torque, the amount of spin density accumulated against the magnetic interfacial through spin Hall effect can be tuned by the ferromagnet's magnetization direction. Indeed, the spin current injection in the ferromagnet is more efficient when the magnetization lies perpendicular to the polarization of the injected spins, i.e. normal to $\mathbf{z} \times \mathbf{j}_{e}$. The modulation of the spin absorption when varying the magnetization direction results in the so-called spin Hall magnetoresistance, which reads [190, 193, 194],

$$
\frac{\Delta \sigma_{x x}}{\sigma_{x x}}=\tilde{\theta}_{\mathrm{sh}}^{2} \frac{\tilde{\lambda}_{\mathrm{sf}}^{\mathrm{N}} \sigma_{\mathrm{N}}}{d_{\mathrm{N}} \sigma_{\mathrm{N}}+d_{\mathrm{F}} \sigma_{\mathrm{F}}} \frac{\tilde{\lambda}_{\mathrm{sf}}^{\mathrm{F}} \sigma_{\mathrm{N}} /\left(\tilde{\lambda}_{\mathrm{sf}}^{\mathrm{N}} \sigma_{\mathrm{F}}\right)-\sigma_{\mathrm{N}} /\left(2 \tilde{\lambda}_{\mathrm{sf}}^{\mathrm{N}} f_{r}^{\uparrow \downarrow}\right)}{\left(1+\tilde{\lambda}_{\mathrm{sf}}^{\mathrm{F}} \sigma_{\mathrm{N}} /\left(\tilde{\lambda}_{\mathrm{sf}}^{\mathrm{N}} \sigma_{\mathrm{F}}\right)\right)\left(1+\sigma_{\mathrm{N}} /\left(2 \tilde{\lambda}_{\mathrm{sf}}^{\mathrm{N}} f_{r}^{\uparrow \downarrow}\right)\right)} .
$$

Remarkably, the spin Hall magnetoresistance displays an angular dependence that is quite different from it bulk counterpart. While bulk anisotropic magnetoresistance of polycrystalline materials is proportional to $\left(\mathbf{m} \cdot \mathbf{j}_{e}\right)^{2}$, the spin Hall magnetoresistance is proportional to $\left[\mathbf{m} \cdot\left(\mathbf{z} \times \mathbf{j}_{e}\right)\right]^{2}$, see Fig. $22(\mathrm{a})-(\mathrm{c})$. This effect has been used recently as an alternative tool to extract the spin Hall angle 
(a)

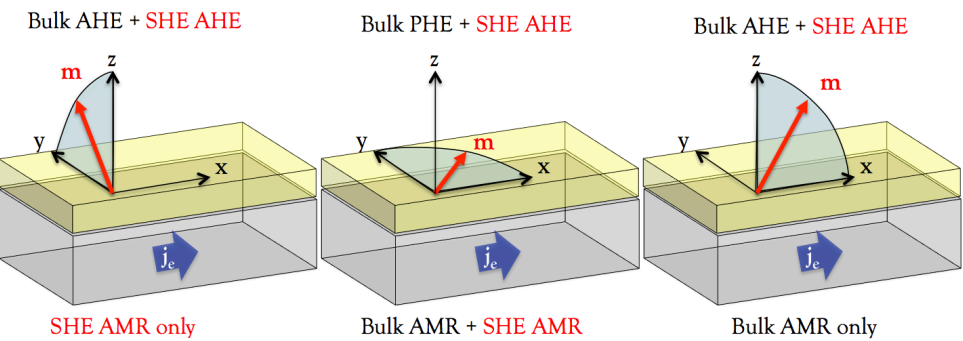

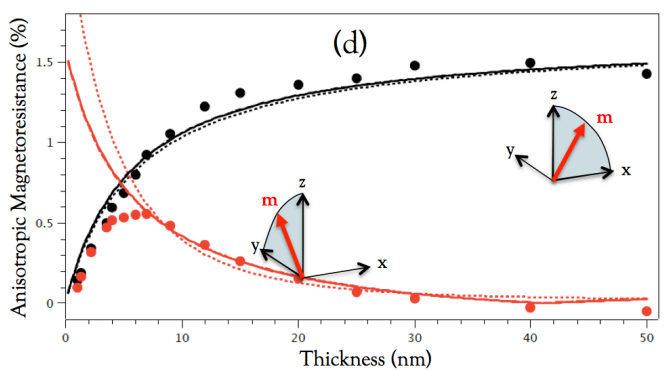

Figure 22: (Color online) Anomalous transport in three different rotation configurations of the magnetization for a bilayer composed of a ferromagnet (yellow layer) deposited on top of a heavy metal (gray layer). Depending on the magnetization rotation plane, the isolated ferromagnet exhibits bulk anomalous Hall effect (AHE), anisotropic magnetoresistance (AMR) or planar Hall effect (PHE). When adjacent to a heavy metal, spin Hall effect (SHE) induces additional effects as described below (a) When the magnetization lies in the $(y, z)$ plane, no bulk magnetoresistance is present and only spin Hall effect-induced magnetoresistance can be detected; (b) When the magnetization lies in the $(x, y)$ plane, both bulk and spin Hall effectinduced magnetoresistance are present; (c) When the magnetization lies in the $(z, x)$ plane, only bulk magnetoresistance is present. Bulk and spin Hall effect-induced anomalous and planar Hall effects have the same angular dependence and therefore cannot be distinguished by angle-dependent measurements. (d) Dependence of the magnetoresistance of the metallic bilayer as a function of the ferromagnetic thickness when the magnetization lies in the $(x, z)$ plane (black lines) and when it lies in the $(y, z)$ plane (red lines), for different noble metal spin diffusion length : $\lambda_{\mathrm{sf}}^{\mathrm{N}}=1.5,5$ and 10nm (dotted, solid and dashes lines). The red and black symbols have been collected from Ref. [199].

in ultrathin bilayers $[195,196,197,194]$. We also emphasize that the combination between spin Hall and inverse spin Hall effect produces an additional Hall voltage, the "spin Hall-induced Hall effect" via the imaginary part of the mixing conductance. One can compute this effect within the drift-diffusion model (not shown), but in general it remains one to two orders of magnitude smaller than the spin Hall magnetoresistance [198]. Figure 22(d) shows an example of theoretical fit using Eq. (24) for a $\mathrm{Pt} / \mathrm{Co} / \mathrm{Pt}$ sample. It is however noteworthy to mention that several experimental and theoretical studies have suggested that other effects might lead to a very similar magnetoresistance phenomena [199, 200, 201], that can be attributed to Rashba spin-orbit coupling [202, 203] or semiclassical size effects [204]. The debate is not settled and we do not intend to further discuss it. 
(a) Diffusive regime

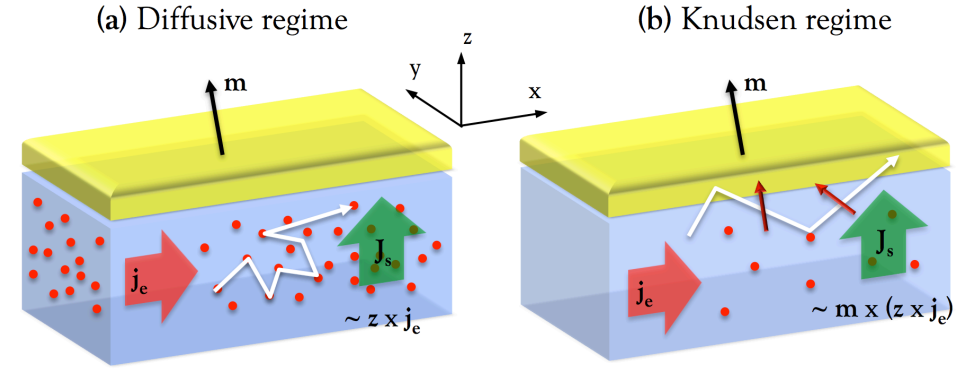

Figure 23: (Color online) Schematics of (a) spin Hall and (b) spin swapping effects in a bilayer composed of a normal metal (blue) and a ferromagnet (yellow) with magnetization $\mathbf{m}$ in the diffusive and Knudsen regimes, respectively. The charge current $\mathbf{j}_{e}$ is injected in the plane of the layers and results in a spin current $\mathbf{J}_{s}$ flowing perpendicular to the interface. Depending on the regime, this spin current induces either (a) a damping-like torque $\sim \mathbf{m} \times[(\mathbf{z} \times \mathbf{j} e) \times \mathbf{m}]$, or (b) a field-like torque $\sim \mathbf{m} \times\left(\mathbf{z} \times \mathbf{j}_{e}\right)$. From [136].

The drift-diffusion model presented above is very practical as it provides explicit analytical formulae for the spin torque and magnetoresistance. However, it is limited to diffusive systems, i.e. bilayers whose thickness is much larger than the mean free path, such that the linear momentum memory is lost. Such an assumption is invalid in most experimentally relevant transition metal bilayers. To model these systems properly, one needs to treat the momentum-resolved transport. One way is for instance to use a Boltzmann description that tracks the non-equilibrium momentum distribution in the system [132, 198]. Another approach is to use a tight-binding model [136].

Consider a metallic bilayer composed of a spin-orbit coupled normal metal and a ferromagnet without spin-orbit coupling [see Fig. 23]. In the limit where the thickness of the normal metal is comparable to the mean free path, the spin Hall effect and spin swapping give rise to two different torques. The former generates a spin current that flows along the normal to the interface $\mathbf{z}$ with a spin polarization along $\left(\mathbf{z} \times \mathbf{j}_{e}\right)$ and results in a damping-like torque on the form $\sim \mathbf{m} \times\left[\left(\mathbf{z} \times \mathbf{j}_{e}\right) \times \mathbf{m}\right]$, see Fig. 23(a). In addition, electrons flowing in the ferromagnet acquire a spin polarization along $\mathbf{m}$ and may scatter towards the normal metal. Once in the normal metal, these electrons experience spin swapping: upon scattering on spin-orbit coupled impurities, they experience a spin-orbit field oriented normal to the scattering plane [i.e. along $(\mathbf{z} \times \mathbf{j} e)]$ and about which their spin precess $[184,167,136]$. Upon this reorientation, a spin current polarized along $\mathbf{m} \times\left(\mathbf{z} \times \mathbf{j}_{e}\right)$ is injected into the ferromagnet and induces a field-like torque, see Fig. 23(b), even in the absence of inverse spin galvanic effect. This effect only survives as long as the linear momentum is well defined, i.e. in a regime where the thickness of the normal metal is comparable to the mean free path. This regime is called the Knudsen regime. When the mean free path becomes much smaller than the normal metal thickness, the system enters the diffusive regime and Eqs. (21)-(22) apply.

So, in summary, as long as the thickness of the normal metal is comparable 
to the mean free path, one should obtain a torque of the form

$$
\boldsymbol{\tau}=\tau_{\mathrm{sh}} \mathbf{m} \times\left[\left(\mathbf{z} \times \mathbf{j}_{e}\right) \times \mathbf{m}\right]+\tau_{\mathrm{sw}} \mathbf{m} \times\left(\mathbf{z} \times \mathbf{j}_{e}\right),
$$

which is quite different from the torques obtained in the diffusive regime [see Eqs. (21)-(22)]. These two effects dominate in distinct disorder regimes: spin Hall effect necessitates strong disorder, while spin swapping survives even for weak disorder. Hence, the nature of the torque should dramatically change from one regime to the other [136]. To confirm this phenomenological picture, we computed the spin transport in a magnetic bilayer using a tight-binding model similar to the one presented in the previous Section [205, 167]. In the present case, instead of injecting the current perpendicular to the interface (Fig. 21 ), the current is now injected along the interface. We calculate the nonequilibrium spin density in the ferromagnet $\delta \mathbf{S}$ and compute the torque $\boldsymbol{\tau}=$ $(2 \Delta / \hbar) \mathbf{m} \times \delta \mathbf{S}$, which is then parsed between damping-like $\left(\tau_{\mathrm{DL}}\right)$ and field-like $\left(\tau_{\mathrm{FL}}\right)$ components. Figure 24 displays the ratio $\tau_{\mathrm{DL}} / \tau_{\mathrm{FL}}$ as a function of disorder and spin-orbit coupling strengths. We find that the torque is dominated by the field-like component in the weak disorder/weak spin-orbit coupling regime, while it is dominated by the damping-like component in the strong disorder/strong spin-orbit coupling regime. When spin Hall effect dominates (strong disorder regime) the torque is mostly damping-like, $\tau_{\mathrm{DL}}>\tau_{\mathrm{FL}}$, and when spin swapping dominates (weak disorder, Knudsen regime) the torque is mostly field-like, $\tau_{\mathrm{FL}}>$ $\tau_{\mathrm{DL}}$.

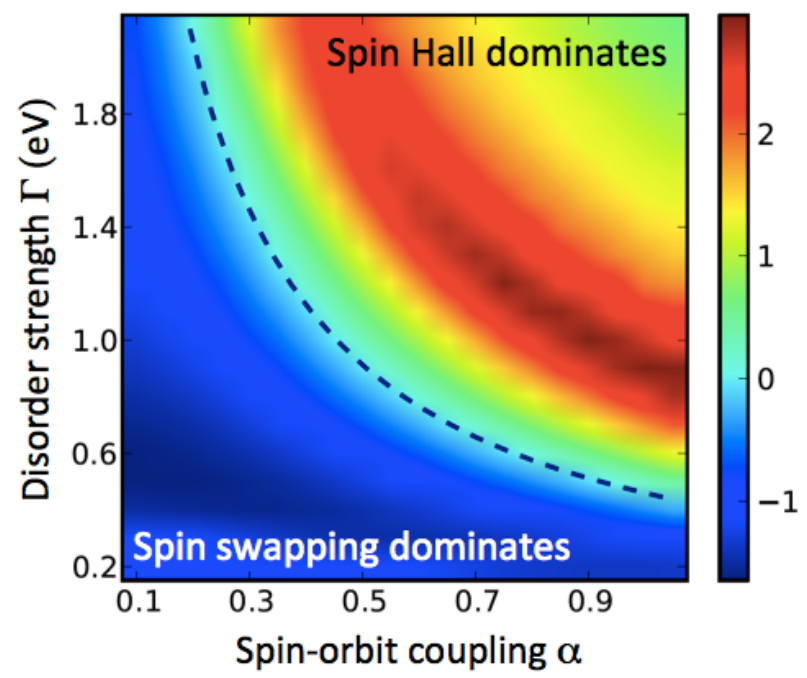

Figure 24: (Color online) Ratio between the magnitude of the field-like torque and dampinglike torque, $\tau_{\mathrm{DL}} / \tau_{\mathrm{FL}}$, as a function of $\Gamma$ and $\alpha$. The ratio is given in logarithmic scale and the dashed line indicates $\tau_{\mathrm{DL}} / \tau_{\mathrm{FL}}=1$. From [136].

These simulations demonstrate that in ultrathin bilayers field-like torques can emerge from spin-dependent scattering in the normal metal. A necessary 
condition is that the thickness of the normal metal must be of the order of the mean free path. This is in fact realized in most of the experiments on spin-orbit torques (but also spin Seebeck effect and spin pumping) where the magnetic multilayers have thicknesses from $10 \mathrm{~nm}$ down to less than $1 \mathrm{~nm}[114,206,207$, 208]. Since the grain size ranges from 5 to $10 \mathrm{~nm}$ in sputtered thin films, the transport is not expected to be diffusive and extrinsic spin swapping could lead to sizable field-like torque (even in the absence of interfacial inverse spin galvanic effect - see below). Magnetic bilayer involving a light metal doped with heavy elements, such as $\mathrm{Cu}(\mathrm{Bi}), \mathrm{Cu}(\mathrm{Ir})$ or $\mathrm{Ag}(\mathrm{Au})$, could be an interesting candidate to observe spin-swapping torque. These materials display large extrinsic spinorbit coupled scattering [176, 177], and hence extrinsic spin swapping, together with ensuring the absence of inverse spin galvanic effect. Recent experiments on $\mathrm{Cu}-\mathrm{Au}$ alloys have reported unconventional temperature-dependence of both damping-like and field-like torque components. This observation supports the presence of extrinsic, disorder-driven spin-charge conversion mechanisms taking place in the $\mathrm{Cu}-\mathrm{Au}$ alloy and could be an indication of the presence of spin swapping [116].

\subsection{Inverse spin galvanic effect}

The second class of mechanisms that leads to spin-orbit torques in magnetic multilayers is the inverse spin galvanic effect at the interface with the ferromagnet. This effect has been modeled by several authors using the toy model of the magnetic Rashba gas [129, 130, 209, 133, 210, 211, 202, 134, 135, 212], and magnetic Dirac gas $[213,68,214,215,216,217]$. These models are quite convenient as they are analytically solvable. Several techniques can be used, such as semiclassical Boltzmann formula, quantum kinetics or Kubo formulae. For instance, in translationally invariant systems, the non-equilibrium spin density induced by an external electric field reads

$$
\begin{aligned}
\delta \mathbf{S}= & \frac{\hbar}{2 \pi \Omega} \int d \epsilon \partial_{\epsilon} f(\epsilon) \operatorname{Tr}\left[\hat{\boldsymbol{\varsigma}} \hat{G}_{\epsilon}^{R}(\hat{\mathbf{v}} \cdot e \mathbf{E})\left(\hat{G}_{\epsilon}^{A}-\hat{G}_{\epsilon}^{R}\right)\right] \\
& +\frac{\hbar}{2 \pi \Omega} \Re \int d \epsilon f(\epsilon) \operatorname{Tr}\left[\hat{\boldsymbol{\varsigma}} \partial_{\epsilon} \hat{G}_{\epsilon}^{R}(\hat{\mathbf{v}} \cdot e \mathbf{E}) \hat{G}_{\epsilon}^{R}-\hat{\boldsymbol{\varsigma}} \hat{G}_{\epsilon}^{R}(\hat{\mathbf{v}} \cdot e \mathbf{E}) \partial_{\epsilon} \hat{G}_{\epsilon}^{R}\right] .
\end{aligned}
$$

Here, $\hat{G}_{\epsilon}^{R(A)}$ is the perturbed retarded (advanced) Green's function, $f(\epsilon)$ is Fermi-Dirac distribution, $\hat{\mathbf{v}}$ is the velocity operator, $\mathbf{E}$ the external electric field and $\hat{\boldsymbol{\varsigma}}$ is the vertex-corrected spin operator. The non-equilibrium spin density possesses three contributions that account for Fermi surface and Fermi sea electrons. It turns out that the first term, $\sim \operatorname{Tr}\left[\hat{\boldsymbol{s}} \hat{G}_{\epsilon}^{R}(\hat{\mathbf{v}} \cdot e \mathbf{E}) \hat{G}_{\epsilon}^{A}\right]$, dominates in the weakly disordered metallic regime. In the limit of weak spin-independent constant energy broadening, $\Gamma \rightarrow 0$, one can parse this term into two main contributions [135] 


$$
\begin{aligned}
\delta \mathbf{S}^{\text {intra }} & =\frac{\hbar}{2 \Gamma \Omega} \sum_{n, \mathbf{k}}\langle n, \mathbf{k}|\hat{\boldsymbol{\zeta}}| n, \mathbf{k}\rangle\langle n, \mathbf{k}|\hat{\mathbf{v}} \cdot e \mathbf{E}| n, \mathbf{k}\rangle \delta\left(\epsilon_{n, \mathbf{k}}-\epsilon_{\mathrm{F}}\right) \\
\delta \mathbf{S}^{\text {inter }} & =\frac{\hbar}{\Omega} \Im \sum_{n, n^{\prime}, \mathbf{k}}\left\langle n, \mathbf{k}|\hat{\boldsymbol{\zeta}}| n^{\prime}, \mathbf{k}\right\rangle\left\langle n^{\prime}, \mathbf{k}|\hat{\mathbf{v}} \cdot e \mathbf{E}| n, \mathbf{k}\right\rangle \frac{f_{n, \mathbf{k}}-f_{n^{\prime}, \mathbf{k}}}{\left(\epsilon_{n, \mathbf{k}}-\epsilon_{n^{\prime}, \mathbf{k}}\right)^{2}} .
\end{aligned}
$$

where $n$ and $n^{\prime}$ refer to eigenstates of the unperturbed Hamiltonian, and $\Im$ takes the imaginary part. The first term involves only transitions within a same band, while the second term involves transition between different bands [135, 218]. Since the latter arises from the perturbation of the eigenstates in the presence of electric field, it is inversely proportional to the energy difference between different bands $\sim 1 /\left(\epsilon_{n, \mathbf{k}}-\epsilon_{n^{\prime}, \mathbf{k}}\right)^{2}$, similarly to the intrinsic spin Hall effect given in Eq. (17). Furthermore, the intraband contribution is proportional to $1 / \Gamma$, i.e. to the current density, while the interband contribution is independent of disorder in this limit and can be related to the Berry curvature in mixed spin-momentum space [112].

The distinction between these two terms is not purely technical, it has important implications in terms of spin-orbit torques. As a matter of fact, each contribution produces a different torque. If one applies time inversion,

$$
\begin{aligned}
& \left\langle n, \mathbf{k}|\hat{\boldsymbol{\zeta}}| n^{\prime}, \mathbf{k}\right\rangle \rightarrow-\left(\left\langle n, \mathbf{k}|\hat{\boldsymbol{\kappa}}| n^{\prime}, \mathbf{k}\right\rangle\right)^{*}, \\
& \left\langle n^{\prime}, \mathbf{k}|\hat{\mathbf{v}} \cdot e \mathbf{E}| n, \mathbf{k}\right\rangle \rightarrow-\left(\left\langle n^{\prime}, \mathbf{k}|\hat{\mathbf{v}} \cdot e \mathbf{E}| n, \mathbf{k}\right\rangle\right)^{*},
\end{aligned}
$$

we see that $\delta \mathbf{S}^{\text {intra }}$ is even, while $\delta \mathbf{S}^{\text {inter }}$ is odd [218]. In other words,

$$
\begin{aligned}
& \delta \mathbf{S}^{\text {intra }}(\mathbf{m})=\delta \mathbf{S}^{\text {intra }}(-\mathbf{m}), \\
& \delta \mathbf{S}^{\text {inter }}(\mathbf{m})=-\delta \mathbf{S}^{\text {inter }}(-\mathbf{m}) .
\end{aligned}
$$

As a result, the intraband contribution produces a torque that is odd as a function of the magnetization direction, while the interband contribution produces a torque that is even upon magnetization reversal. These two types of torques are referred to as field-like and damping-like, respectively. More specifically, in the case of a two dimensional electron gas with both magnetism and Rashba spin-orbit coupling, one obtains

$$
\boldsymbol{\tau}_{\mathrm{R}}=(2 \Delta / \hbar) \mathbf{m} \times \delta \mathbf{S}=\tau_{\mathrm{FL}} \mathbf{m} \times(\mathbf{z} \times e \mathbf{E})+\tau_{\mathrm{DL}} \mathbf{m} \times[(\mathbf{z} \times e \mathbf{E}) \times \mathbf{m}],
$$

the first term being attributed to intraband contribution, while the second is due to the interband contribution [133, 135, 134, 212]. The latter possesses exactly the same form as the damping-like torque coming from spin Hall effect and, as we shall see below, it is often hard to distinguish between them, even theoretically. Interestingly, in the case of the magnetic surface of topological insulators described by the massive Dirac Hamiltonian, the torque becomes $[217]$

$$
\boldsymbol{\tau}_{\mathrm{D}}=\tau_{\mathrm{FL}} \mathbf{m} \times(\mathbf{z} \times e \mathbf{E})+\tau_{\mathrm{DL}} m_{z} \mathbf{m} \times e \mathbf{E} .
$$


While the field-like torque remains the same as in the Rashba gas, the dampinglike torque now vanishes when the magnetization lies in the plane of the interface, a mechanism that is specific to topological insulators but can also be recovered in the strong Rashba limit for two dimensional magnetic Rashba gases [135].

Unfortunately, the Rashba model used in many of the recent studies of spinorbit torques presents major flaws that limit its quantitative predictive power. First, as already mentioned, it formally applies only to two dimensional electron gases with sharp interfaces and completely miss the interfacial orbital physics. Second, its simplicity leads to pathological behaviors that have been put forward recently in the context of intrinsic spin Hall effect and Rashba torque. As a matter of fact, both intrinsic Rashba spin Hall effect [179, 219] and damping-like Rashba torque [212] vanish when taking into account vertex corrections, due to the unrealistically simple energy dispersion of the gas. To add to the confusion, Ado et al. [220, 221] recently demonstrated that the first Born approximation itself is insufficient to compute the intrinsic non-equilibrium properties, and that one should add higher order terms in the diagrammatic expansion (the authors specifically studied the $X$ and $\Psi$ diagrams). These studies emphasize the subtleties of the treatment of impurity self-averaging in these simple models. One way to circumvent these difficulties is to consider realistic systems, where the band dispersion is much more complicated than the simple free-electron parabolic dispersion (or than the infinite Dirac cone, in the case of topological insulator surfaces).

\subsubsection{Inverse spin galvanic effect at realistic interfaces}

Recently, Haney et al. [132] have computed the current-driven field-like torque in $\mathrm{Pt} / \mathrm{Co}(111)$. The ab initio method is based on a linear combination of spherical orbitals and the current and torque are calculated within the relaxation time approximation. This approach disregards the spin Hall effect or any intrinsic contributions to the inverse spin galvanic effect. These calculations, reported on Fig. 25(a), first show that in spite of the high complexity of the band structure [see Fig. 8(b,c)], sizable inverse spin galvanic effect emerges at the interface between $\mathrm{Pt}$ and Co. The magnitude of the Rashba field (about 100 Oe for $10^{8} \mathrm{~A} / \mathrm{cm}^{2}$ ) is consistent with the experimental observations [223, 109]. Moreover, Fig. 25(a) displays the current-driven Rashba when the induced magnetism of the $\mathrm{Pt}$ is turned off (diamonds) and when the spin-orbit coupling on $\mathrm{Pt}$ is turned off (circles). It appears that while Pt magnetism has a negligible effect on the Rashba field, its spin-orbit coupling is the main driving force, confirming the simple Rashba scenario evoked previously. Nonetheless, even in the absence of Pt spin-orbit coupling, we emphasize that the Rashba field does not vanish since Co spin-orbit coupling is still present. This produces a Rashba field of the same sign but an order of magnitude smaller. Indeed, the sign of the Rashba field is associated with the distortion of the wave function at the interface, which is fixed by the $\mathrm{Co} / \mathrm{Pt}$ slab ordering. Hence, one can speculate that such a torque exists at $\mathrm{Co} / \mathrm{MgO}$ interfaces and can be controlled by oxygen and, by extension, gate voltage, in the same spirit as DMI (see Section 3 and 

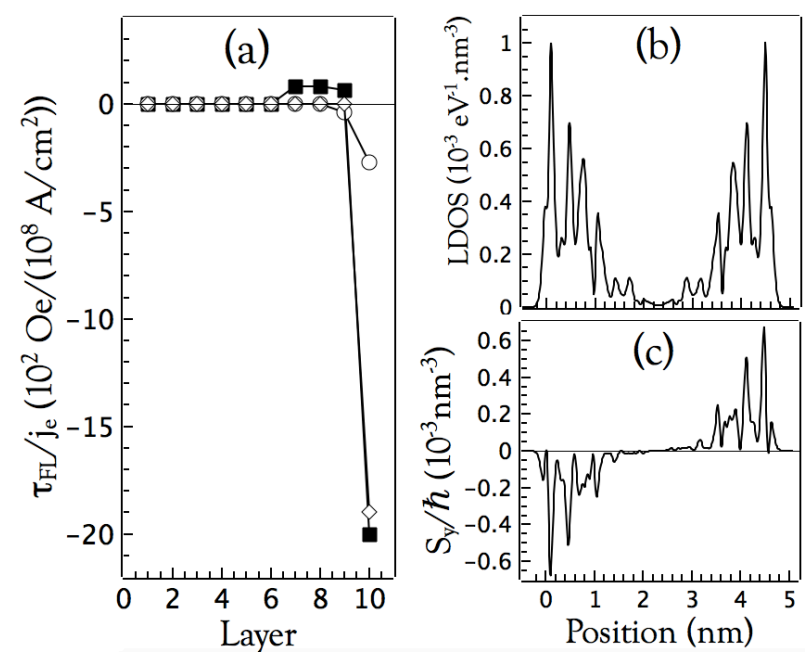

Figure 25: (Color online) (a) Layer-resolved field-like torque in $\mathrm{Pt} / \mathrm{Co}(111)$ (black squares), when turning off the induced magnetization on $\mathrm{Pt}$ (open diamonds) and when turning off the spin-orbit coupling on $\mathrm{Pt}$ (open circles). The slab is composed of 2 Co monolayers (shaded region) deposited on 8 monolayers of Pt. From Ref. [30]. (b) Local density of states and (c) current-driven spin density profile in $\mathrm{B}_{2} \mathrm{Se}_{3}$ slab composed of five quintuple layers. From [222].

Ref. [102]).

The inverse spin galvanic effect has also been investigated from first principles at the surface of $\mathrm{Bi}_{2} \mathrm{Se}_{3}$ using the ATK package [222]. $\mathrm{Bi}_{2} \mathrm{Se}_{3}$ is a topological insulator, namely it is insulating in its bulk and possesses highly conductive, spin-orbit coupled surface states. The spatial profile of the local density of states reported on Fig. 25(b) shows the extension of the surface states over $2 \mathrm{~nm}$ on each side of the slab. Although the system is not magnetic, one can clearly see the buildup of the spin accumulation across the slab, as displayed in Fig. 25(c). We emphasize that the extension of "surface states" is actually very large (2 $\mathrm{nm}$ ) and is comparable to the thicknesses of normal metals in magnetic bilayers (from 1 to $5 \mathrm{~nm}$ ). Therefore, interfacial orbital hybridization and scattering against defects can severely impact the spin physics in this extended region. Considering the recent experimental interest for spin-orbit torques at the magnetic surface of topological insulators [124, 125, 126, 127, 128], more extensive and systematic investigations of such systems are highly desirable.

Freimuth et al. [218, 80, 224] have recently developed an intensive effort towards the comprehensive computation of current-induced torques in magnetic thin films. Based on the full potential linear augmented plane wave method implemented in FLEUR and computing Kubo-Bastin formula, thereby accounting for intrinsic spin Hall torque as well as intrinsic and extrinsic inverse spin galvanic effect, the authors have investigated and characterized the spin-orbit torques in $\mathrm{Pt} / \mathrm{Co}(111), \mathrm{W} / \mathrm{Mn}(001)$ and $\mathrm{L}_{0} \mathrm{FePt} / \mathrm{Pt}$. These calculations con- 

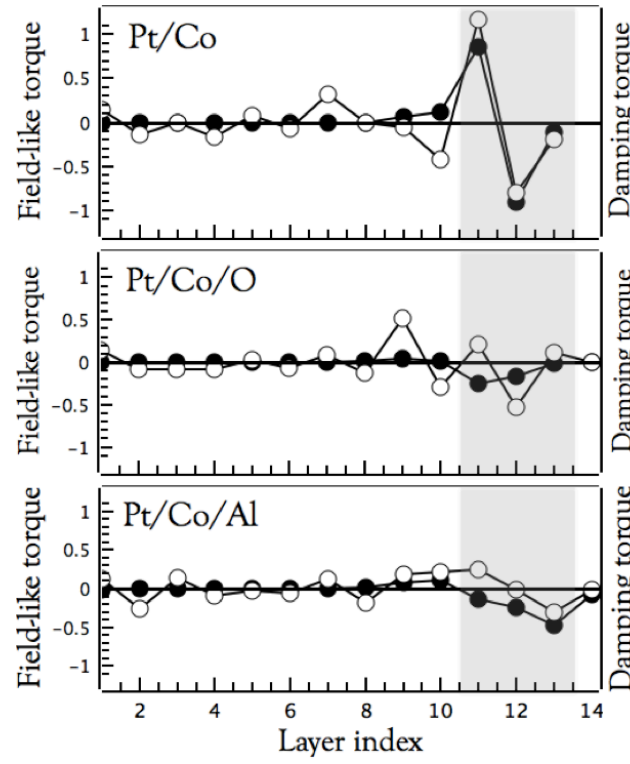

Figure 26: (Color online) Layer-resolved field-like (left panels) and damping-like torques (right panels) in $\mathrm{Pt} / \mathrm{Co}, \mathrm{Pt} / \mathrm{Co} / \mathrm{O}$ and $\mathrm{Pt} / \mathrm{Co} / \mathrm{Al}$ stacks. The torques are represented by the filled symbols, while the spin currents associated with them are represented by open symbols. The Co region is denoted by the shaded area. From Ref. [218].
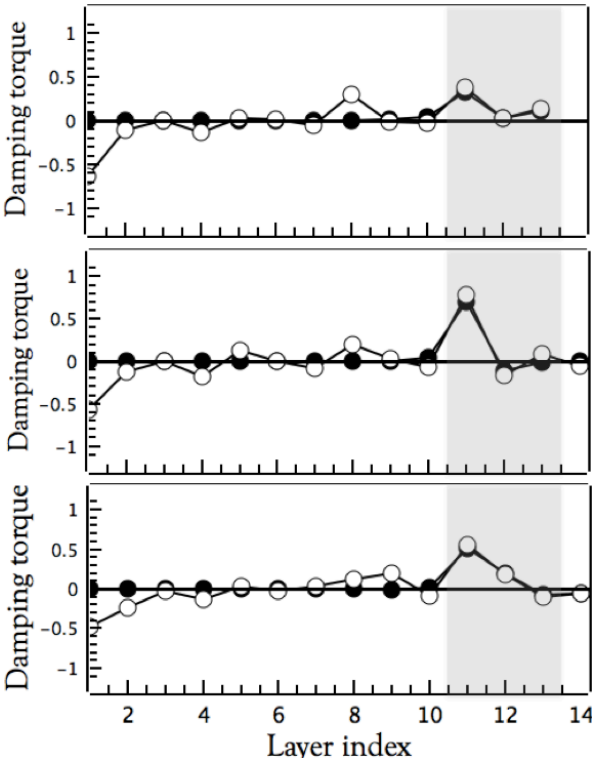


\section{Unconventional dynamics}

In the previous Sections, we have seen that transition metal interfaces encompass several exotic effects enabled by spin-orbit coupling: Rashba-type spin splitting, Dzyaloshinskii-Moriya antisymmetric exchange interaction, and spinorbit torques. As a result, the magnetic texture at these interfaces is likely to be chiral and can be manipulated electrically. In this last Section, we are interested in two aspects of the chiral magnetic textures: their energy dissipation and the additional "topological" torque that flowing currents exert on these textures.

\subsection{Chiral Damping}

\subsubsection{Fundamentals of magnetic damping}

The magnetic damping arises from various mechanisms involving non-linear spin waves processes [225, 226, 227] such as two- $[228,229,230]$ and threemagnon interactions [231] (dominant in magnetic insulators [232]) as well as electronic processes driven by spin relaxation [233, 234, 235] (dominant in metals). The latter mechanisms are of primary interest for us because of the dominant role of spin-orbit coupling. In the hydrodynamic limit, the magnetic damping is a non-local, anisotropic $3 \times 3$ tensor of the form [236]

$$
\left.\partial_{t} \mathbf{m}(\mathbf{r}, t)\right|_{\text {diss }}=\mathbf{m}(\mathbf{r}, t) \times \int d \mathbf{r}^{\prime} \underline{\underline{\alpha}}\left(\mathbf{r}, \mathbf{r}^{\prime}\right) \cdot \partial_{t} \mathbf{m}\left(\mathbf{r}^{\prime}, t\right) .
$$

In an isotropic magnetic material, the dissipation reduces to the well-known Gilbert damping form, $\left.\partial_{t} \mathbf{m}(\mathbf{r}, t)\right|_{\text {diss }}=\alpha \mathbf{m} \times \partial_{t} \mathbf{m}$. However, in magnetic metals presenting a strong (bulk or interfacial) magnetic anisotropy, one can reasonably expect that such anisotropy gets imprinted in the magnetic damping constant $[237,3]$. Mills and Rezende [3] showed that in a magnetic material with uniaxial anisotropy axis $\mathbf{z}$, the damping reads

$$
\left.\partial_{t} \mathbf{m}\right|_{\text {diss }} ^{z}=\epsilon_{\|} \mathbf{m} \times \mathbf{z} \partial_{t} m_{z}+\epsilon_{\perp} m_{z} \mathbf{z} \times \partial_{t} \mathbf{m} .
$$

This anisotropic damping is derived from general considerations and, in the particular case considered here, only affects the components of the magnetization transverse to the anisotropy direction $\mathbf{z}$. Although not observed yet, this anisotropic damping should be quite large in systems with interfacial perpendicular magnetic anisotropy.

Physically, the mechanism responsible for the magnetic damping in metals can be summarized as follows: the precessing magnetization excites spinorbit coupled electron-hole pairs that relax their own spin angular momentum through momentum relaxation processes via defects, impurities or phonon scattering [234]. This process is usually modeled by the so-called breathing Fermi surface model that relates the damping constant to the angular dependence of the electronic energy, $\partial_{\theta} \epsilon_{n, \mathbf{k}}$, and to the momentum scattering time $\tau$ [238]

$$
\lambda_{\mathrm{BFS}}=\tau \frac{g^{2} \mu_{\mathrm{B}}^{2}}{\hbar^{2} \Omega} \sum_{n, \mathbf{k}}\left(\partial_{\theta} \epsilon_{n, \mathbf{k}}\right)^{2} \delta\left(\epsilon_{n, \mathbf{k}}-\epsilon_{\mathrm{F}}\right) .
$$


The Gilbert damping is simply defined $\alpha=\lambda M_{s} / \gamma$, where $M_{s}$ is the saturation magnetization and $\gamma$ is the gyromagnetic ratio. This formula is valid for $\tau \rightarrow$ $+\infty$ and only considers electrons close to the Fermi level. An equivalent picture was also developed by Kambersky by considering the spin relaxation of long wavelength magnons $(q \rightarrow 0)[239,240]$, yielding the so-called torque correlation formula

$$
\lambda_{\tau}=\frac{g^{2} \mu_{\mathrm{B}}^{2}}{\pi \hbar \Omega}\left(\frac{\hbar}{2 \tau}\right)^{2} \sum_{n, m, \mathbf{k}} \frac{\left|\left\langle n, \mathbf{k}\left|\left[\hat{\sigma}^{-}, \hat{\mathcal{H}}_{\mathrm{so}}\right]\right| m, \mathbf{k}\right\rangle\right|^{2}}{\left[\left(\epsilon_{\mathrm{F}}-\epsilon_{n, \mathbf{k}}\right)^{2}+(\hbar / 2 \tau)^{2}\right]\left[\left(\epsilon_{\mathrm{F}}-\epsilon_{m, \mathbf{k}}\right)^{2}+(\hbar / 2 \tau)^{2}\right]} .
$$

In the limit of weak scattering Eq. (38) reduces to Eq. (37) [234]. Equation (38) is more general since it accounts for both intraband $(\propto \tau)$ and interband contributions $(\propto 1 / \tau)$, and therefore applies to a wider ranger of disorder regime $[241,235]$. When applied to realistic band structure, such as Co, Ni or Fe, the magnetic damping adopts the symmetries of the crystal structure, resulting in rotational and orientational anisotropic damping [242], as illustrated in Fig. 27. The damping anisotropy calculated in these systems can be as large as $50 \%$. One has to recall that the magnetocrystalline anisotropy for these bulk ferromagnets is of the order of a few $\mu \mathrm{eV} /$ atom. One can reasonably speculate that damping anisotropy in ultrathin multilayers with interfacial perpendicular magnetic anisotropy of the order of a few meV/atom should be actually gigantic.

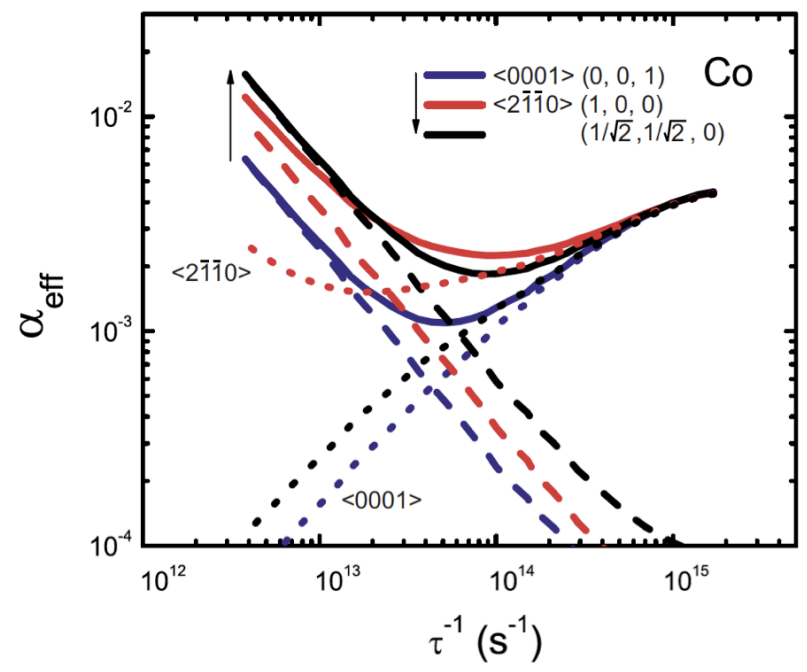

Figure 27: Magnetic damping in bulk Co from first principle calculations, using the effective field approach. The magnitude of the magnetic damping exhibits a significant anisotropy depending on the orientation of the precessing magnetization. In strongly disorder systems $\left(\tau^{-1} \rightarrow+\infty\right)$, this anisotropy vanishes. From Ref. [242].

The two formulae presented above are quite popular but do not account for non-locality. One way to circumvent this limitation is to derive the magnetic damping within the linear response theory (see for instance [243, 244] or the 
Supplementary material of [245]). Let us consider a general Hamiltonian, $\hat{\mathcal{H}}=$ $\hat{\mathcal{H}}_{0}+\Delta \hat{\boldsymbol{\sigma}} \cdot \mathbf{m}$, where the second term connects the itinerant electron spins $\hat{\boldsymbol{\sigma}}$ with the local magnetic moment $\mathbf{m}$. We now introduce a small perturbation to the magnetization $\mathbf{m} \rightarrow \mathbf{m}+\delta \mathbf{m}$ that is time-dependent, $\partial_{t} \delta \mathbf{m} \neq 0$. Hence, the moving magnetization pumps a non-equilibrium spin density $\delta \mathbf{S}=\underline{\chi} \cdot \partial_{t} \mathbf{m}$, which is turns exerts a torque on the local magnetization, $\boldsymbol{\tau}=(2 \Delta / \hbar) \mathbf{m} \times \delta \mathbf{S} \sim$ $\mathbf{m} \times\left[\underline{\underline{\chi}} \cdot \partial_{t} \mathbf{m}\right]$. This torque modifies the dynamics of the magnetization, depending on the symmetry of the tensor $\chi \underline{x}$ upon magnetization reversal. Indeed, one can rewrite $\underline{\underline{\chi}}(\mathbf{m})=\underline{\underline{\chi}}^{\text {odd }}+\underline{\underline{\chi}}^{\text {even }}$, where $\underline{\underline{\chi}}^{\text {odd }}(\mathbf{m})=-\underline{\underline{\chi}}^{\text {odd }}(-\mathbf{m})$ and $\underline{\underline{\chi}}^{\text {even }}(\mathbf{m})=$ $\underline{\underline{\chi}}^{\text {even }}(-\overline{\mathbf{m}})$. As a result, the torque possesses two components, $\overline{\boldsymbol{\tau}}=\boldsymbol{\tau}_{\text {odd }}+$ $\overline{\bar{\tau}}_{\text {even }}$, renormalizing the magnetization precession rate, and inducing a magnetic damping, respectively. From Kubo formula, one obtain $\boldsymbol{\tau}=\mathbf{m} \times \int d^{3} \mathbf{r}^{\prime}\left(\underline{\underline{\alpha}} \cdot \partial_{t} \mathbf{m}\right)$, where the tensor $\underline{\underline{\alpha}}\left(\mathbf{r}, \mathbf{r}^{\prime}\right)$ has the components [244, 245]

$$
\begin{aligned}
\alpha^{i j}\left(\mathbf{r}, \mathbf{r}^{\prime}\right)= & \frac{\Delta^{2}}{\pi \Omega} \Re \int d \epsilon \partial_{\epsilon} f(\epsilon) \operatorname{Tr}\left[\hat{\varsigma}_{i} \hat{G}_{\epsilon}^{R}\left(\mathbf{r}, \mathbf{r}^{\prime}\right) \hat{\sigma}_{j}\left(\hat{G}_{\epsilon}^{A}\left(\mathbf{r}^{\prime}, \mathbf{r}\right)-\hat{G}_{\epsilon}^{R}\left(\mathbf{r}^{\prime}, \mathbf{r}\right)\right)\right] \\
& +\frac{\Delta^{2}}{\pi \Omega} \Re \int d \epsilon f(\epsilon) \operatorname{Tr}\left[\hat{\varsigma}_{i} \partial_{\epsilon} \hat{G}_{\epsilon}^{R}\left(\mathbf{r}, \mathbf{r}^{\prime}\right) \hat{\sigma}_{j} \hat{G}_{\epsilon}^{R}\left(\mathbf{r}^{\prime}, \mathbf{r}\right)-\hat{\varsigma}_{i} \hat{G}_{\epsilon}^{R}\left(\mathbf{r}, \mathbf{r}^{\prime}\right) \hat{\sigma}_{j} \partial_{\epsilon} \hat{G}_{\epsilon}^{R}\left(\mathbf{r}^{\prime}, \mathbf{r}\right)\right] .
\end{aligned}
$$

The vertex correction included in $\hat{\boldsymbol{\varsigma}}$ is crucial to ensure the proper treatment of the 1st Born approximation [244]. Similarly to the spin-orbit torques discussed in the previous Section, Eq. (26), the non-local magnetic damping possesses a Fermi surface contribution as well as a Fermi sea contribution. This formula is quite convenient to compute the non-local magnetic damping, including its chiral contribution, although no such calculation has been performed yet, to the best of our knowledge. Notice finally that in the case of the magnetic surface of topological insulators, the magnetic damping is directly proportional to the conductivity tensor. Indeed, since $\hat{\mathcal{H}}=v \mathbf{p} \cdot(\mathbf{z} \times \hat{\boldsymbol{\sigma}}), \hat{\mathbf{v}}=\partial_{\mathbf{p}} \hat{\mathcal{H}}=v(\mathbf{z} \times \hat{\boldsymbol{\sigma}})$, establishing a direct equivalence between spin dynamics (spin-spin correlation) and charge dynamics (current-current correlation).

Another source of magnetic damping in thin films is the spin pumping originally identified by Tserkovnyak et al. [246, 247]. In this mechanism, a precessing magnetization pumps a spin current out of the magnetic system, through an interface for instance. This spin current, $\left.\mathcal{J}_{s}\right|_{\text {interf }}=\hbar\left(g^{\uparrow \downarrow} / 4 \pi\right) \mathbf{m} \times \partial_{t} \mathbf{m}$, is then absorbed by the adjacent layer through spin relaxation. As a reaction, the magnetic damping of the ferromagnet is enhanced [248, 249, 250, 251, 252]. Concretely, the spin pumping contribution to the magnetic damping reads $\alpha_{\mathrm{sp}}=$ $\mathcal{A} g^{\uparrow \downarrow}$, where $g^{\uparrow \downarrow}$ is the real part of the spin mixing conductance and $\mathcal{A}$ is a coefficient that accounts for the spin current backflow in the adjacent metals and that vanishes in the limit of infinite spin diffusion length. The concept of spin pumping has been recently extended to the case of time-dependent magnetic textures $[253,254,255,256,36]$. When a magnetic texture evolves in time, spin pumping acts locally so that itinerant electrons experience a spin-dependent emergent electromagnetic field induced by the texture. This emergent electromagnetic field is discussed in more details in Section 5.2. Zhang and Zhang 
recently showed that this field contributes to energy dissipation in magnetic textures [257]. In this case, the damping adopts a tensorial form, $\boldsymbol{\tau}=\mathbf{m} \times \mathcal{D} \cdot \partial_{t} \mathbf{m}$, where

$$
\mathcal{D}_{i j}=\xi \sum_{k}\left(\mathbf{m} \times \partial_{k} \mathbf{m}\right)_{i}\left(\mathbf{m} \times \partial_{k} \mathbf{m}\right)_{j},
$$

and $\xi$ is a material dependent parameter $\left(\xi \sim 0.5-1 \mathrm{~nm}^{2}\right)$. This additional damping is second order in the spatial gradient of the magnetization and therefore expected to be only significant in sharp magnetic textures such as vortices or skyrmions.

\subsubsection{Phenomenological theory of chiral damping}

Most of the theories of magnetic damping developed to date assume the long wavelength limit $(q \rightarrow 0)$. Since the energy relaxation rate of spin waves depends on their wave vector (the higher the spin wave energy, the stronger its dissipation), one expects that the energy dissipation of magnetic textures should depend on magnetization gradient [see Eq. (40)]. As discussed above, in magnets with inversion symmetry, the magnetic damping is enhanced by a corrective term of the order of $1 / \lambda_{\mathrm{dw}}^{2}$, where $\lambda_{\mathrm{dw}}$ is the magnetic exchange length. However, in magnetic systems lacking inversion symmetry such as the systems in which Dzyaloshinskii-Moriya is observed, the energy dissipation becomes linear in the magnetization gradient and may depend on the chirality of the magnetic texture.

As a matter of fact, in the limit of smooth textures, the tensorial components of the damping is a function of the magnetization direction and of its spatial gradients, $\alpha^{i j}=\alpha^{i j}(\mathbf{m}, \nabla \mathbf{m})$, such that, to the first order in magnetization gradient [258]

$$
\alpha^{i j}=\alpha_{0}^{i j}+\sum_{l m} K_{l m}^{i j} m_{l} m_{m}+\sum_{k l m} L_{k l m}^{i j} m_{k} \partial_{l} m_{m} .
$$

The first term is the isotropic damping, the second term amounts for the anisotropic contribution and the third term is the chiral damping. Only terms bilinear in magnetization direction $m_{i}$, i.e. even under time reversal symmetry, are retained in the expansion. In systems possessing inversion symmetry, the third term of Eq. (41) vanishes, $L_{k l m}^{i j}=L_{m l k}^{i j}$. However, where inversion symmetry is broken, $L_{k l m}^{i j}=-L_{m l k}^{i j}$, and this term reduces to Lifshitz antisymmetric invariants, $\propto m_{k} \partial_{l} m_{m}-m_{m} \partial_{l} m_{k}$. This functional dependence is similar to the Dzyaloshinskii-Moriya interaction: since both effects are bilinear in magnetization and fulfill Neumann's principle, they adopt the same general form. In addition though, Onsager reciprocity imposes $\alpha^{i j}=\alpha^{j i}$, and hence, one can construct a chiral damping up to the linear order in magnetization gradient based on symmetry arguments. For instance, in a cubic system with bulk spatial inversion symmetry breaking, the chiral damping becomes

$$
\alpha^{i j}=\alpha_{0}^{i j}+\alpha_{3 d}^{i j} \lambda_{\mathrm{dw}} \mathbf{m} \cdot(\boldsymbol{\nabla} \times \mathbf{m}),
$$


where $\lambda_{\mathrm{dw}}$ defines the characteristic exchange length. In a two-dimensional system with interfacial symmetry breaking along $\mathbf{z}$, i.e. invariant under $\mathcal{C}_{\infty z}$ rotation symmetry, the damping takes the form

$$
\left.\alpha^{i j}=\alpha_{0}^{i j}+\alpha_{z}^{i j} \lambda_{\mathrm{dw}} \mathbf{m} \cdot[(\mathbf{z} \times \boldsymbol{\nabla}) \times \mathbf{m})\right],
$$

Other forms of Dzyaloshinskii-Moriya interaction have been derived in the case of magnetic interfaces for instance [259].

\subsubsection{Microscopic origin of chiral damping}

As discussed in Section 4, there are two manners to exert a torque on a magnetic order parameter: either by generating a non-equilibrium spin density $\delta \mathbf{S}$, or by injecting a spin current in the magnet $\mathcal{J}_{s}$. In the first case, the torque reads $\boldsymbol{\tau}=(2 \Delta / \hbar) \mathbf{m} \times \delta \mathbf{S}$, while in the second case it is associated with the spatial gradient of the spin current $\boldsymbol{\tau}=-\boldsymbol{\nabla} \cdot \mathcal{J}_{s}$. These two pictures are equivalent in the absence of spin-orbit coupling, but may lead to different torques when spin-orbit is present. We present below four phenomena that can lead to chiral magnetic damping.

Rashba spin-orbit coupling. In ferromagnets with interfacial Rashba spin-orbit coupling, it has been shown that the magnetic damping adopts the form of a tensor linear in both magnetization gradient and Rashba strength [206, 202] [see Fig. 28(a)]. Two distinct mechanisms can be identified. First, as already mentioned, a time-dependent magnetic texture generates an effective spindependent electric field on the itinerant electrons, producing a charge current $j_{e, i} \sim \mathbf{m} \cdot\left(\partial_{i} \mathbf{m} \times \partial_{t} \mathbf{m}\right)$. Through inverse spin galvanic effect, this charge current induces a spin density that exerts a torque on the magnetization $\tau \sim$ $\alpha_{\mathrm{R}} \mathbf{m} \times\left(\mathbf{z} \times \mathbf{j}_{e}\right)$, where $\alpha_{\mathrm{R}}$ is the Rashba parameter. A symmetric effect coexists: the precessing magnetization pumps a spin-polarized current through spin galvanic effect, $\mathcal{J}_{s} \sim \alpha_{\mathrm{R}} \mathbf{m} \otimes \mathbf{z} \times \partial_{t} \mathbf{m}$ (we remind that the spin current is a tensor in the spin $\otimes$ real spaces). The corresponding spin current is then absorbed by the magnetic texture through $\tau=-\nabla \cdot \mathcal{J}_{s}$. As a result, the total magnetic damping is linear in Rashba parameter, as well as in magnetization gradient, and $[206,202,260]$

$$
\boldsymbol{\tau} \sim\left[\left(\mathbf{z} \times \partial_{t} \mathbf{m}\right) \cdot \boldsymbol{\nabla}\right] \mathbf{m}-(\mathbf{z} \times \boldsymbol{\nabla}) \times\left(\mathbf{m} \times \partial_{t} \mathbf{m}\right) .
$$

$s$-d antisymmetric interaction. As demonstrated in Ref. [70], in transition metal ferromagnets both localized ( $p d$ hybridized) and delocalized electrons (spd hybridized) contribute to DMI and therefore one can parse the total spin moment $\mathbf{S}_{i}$ into localized ( $d$-dominated) and delocalized ( $s$-dominated) contributions $\mathbf{S}_{i}=\mathbf{S}_{i}^{d}+\hat{\mathbf{s}}_{i}^{s}$. The resulting DMI between sites $i$ and $j$ can be phenomenologically rewritten upto the linear order in $\hat{\mathbf{s}}^{s}$ as $\mathbf{D}_{i j} \cdot \mathbf{S}_{i} \times \mathbf{S}_{j}=$ $\mathbf{D}_{i j}^{d d} \cdot \mathbf{S}_{i}^{d} \times \mathbf{S}_{j}^{d}+\mathbf{D}_{i j}^{s d} \cdot \mathbf{S}_{i}^{d} \times \hat{\mathbf{s}}_{j}^{s}$ (neglecting a higher order term $\sim \hat{\mathbf{s}}_{i}^{s} \times \hat{\mathbf{s}}_{j}^{s}$ ). The first term only involves orbital overlap between localized states while the second term describes the chiral exchange between the local and itinerant spins. In the 
continuous limit, the spin-orbit coupling Hamiltonian of the itinerant electrons can be written as

$$
\hat{\mathcal{H}}_{\mathrm{sd}}=\frac{\alpha_{\mathrm{D}}}{\hbar} \hat{\boldsymbol{\sigma}} \cdot[(\mathbf{z} \times \hat{\mathbf{p}}) \times \mathbf{m}],
$$

where $\alpha_{\mathrm{D}}=D_{\mathrm{sd}} a$ ( $a$ is the lattice parameter) is the strength of the $s$ - $d$ DM interaction. Equation (45) suggests that itinerant spins flowing through the magnetic texture illustrated on Fig. 28(b) experience both the inhomogeneous exchange field $\sim \mathbf{m}$, and the inhomogeneous, texture-dependent $s$ - $d$ DM field $\sim(\mathbf{z} \times \boldsymbol{\nabla}) \times \mathbf{m}$. The cooperation of these two terms introduces a distortion of the Fermi surface that depends on the magnetization gradient and therefore promotes chiral damping. Within the relaxation time approximation, one obtains a damping torque [260]

$$
\boldsymbol{\tau} \sim\left\{[(\mathbf{z} \times \boldsymbol{\nabla}) \times \mathbf{m}] \times\left(\mathbf{m} \times \partial_{t} \mathbf{m}\right)\right\}_{\perp},
$$

where the subscript $\perp$ refers to the part that is transverse to the magnetization. Notice that the chiral damping arising from $s-d$ DMI $\left(\propto D_{\text {sd }}\right)$ does not necessarily scale with the DMI itself $\left(\propto D_{\mathrm{dd}}\right)$ as both effects, chiral damping and asymmetric exchange, involve different electron orbitals.

Anomalous spin pumping. This mechanism is illustrated in Fig. 28(c) and arises from the interplay between spin motive force and anomalous Hall effect. As mentioned above, time-dependent spin textures generate a local charge current $j_{e, i} \sim \mathbf{m} \cdot\left(\partial_{i} \mathbf{m} \times \partial_{t} \mathbf{m}\right)$. When anomalous Hall effect is present in the ferromagnet, this charge current $\mathbf{j}_{e}$ can be converted into a spin Hall current of the form $e \mathcal{J}_{s, i}=\theta_{\mathrm{sh}} \mathbf{m} \otimes \mathbf{m} \times \mathbf{j}_{e}$, i.e. flowing along the direction $\mathbf{m} \times \mathbf{j}_{e}$ and spin polarized along $\mathbf{m}$. If this spin Hall current can be injected out of the ferromagnet into an adjacent spin sink, it exerts a dissipative torque on the magnetization, similarly to the well-known spin pumping mechanism [246]. Concretely, considering a domain wall along $\mathbf{x}$ deposited on a normal metal with an interface normal to $\mathbf{z}$ [Fig. 28(c)], as long as the magnetization $\mathbf{m}$ has a non-zero component along $\mathbf{y}$, the spin Hall current that is injected in the adjacent metal along $\mathbf{z}$ is spin polarized along $\mathbf{y}$ and exerts the torque

$$
\boldsymbol{\tau} \sim\left[\left(\partial_{x} \mathbf{m} \times \partial_{t} \mathbf{m}\right) \cdot \mathbf{y}\right] \mathbf{m} \times(\mathbf{y} \times \mathbf{m}) .
$$

This damping torque vanishes when the wall is either in Bloch $(\varphi=\pi / 2)$ or Néel configuration $(\varphi=0)$ and is maximum in-between.

Spin swapping. The last mechanism is spin swapping, discussed in Section 4 and illustrated in Fig. 28(d). A precessing magnetization pumps a spin current, polarized along $\mathcal{J}_{s, z}^{\text {pump }} \sim \mathbf{m} \times \partial_{t} \mathbf{m} \otimes \mathbf{z}$, into the normal metal [246]. Such a spin current can be converted into a charge current through inverse spin Hall effect [261], but also enhances the magnetic damping of the ferromagnet [246]. Upon spin swapping this pumped spin current is converted into another spin current $\mathcal{J}_{s, z}^{\mathrm{sw}} \sim \mathbf{y} \times\left(\mathbf{m} \times \partial_{t} \mathbf{m}\right) \otimes \mathbf{z}(\mathbf{y}$ being the direction of the spin-orbit field perpendicular to the scattering plane). While this new spin current does 

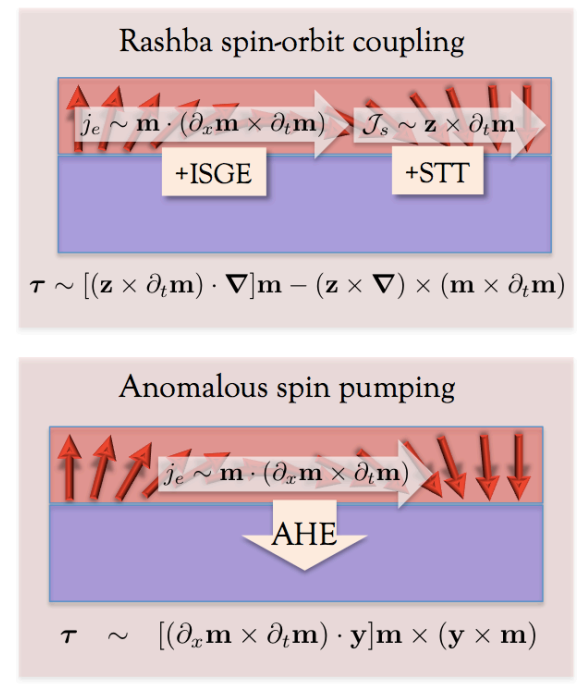
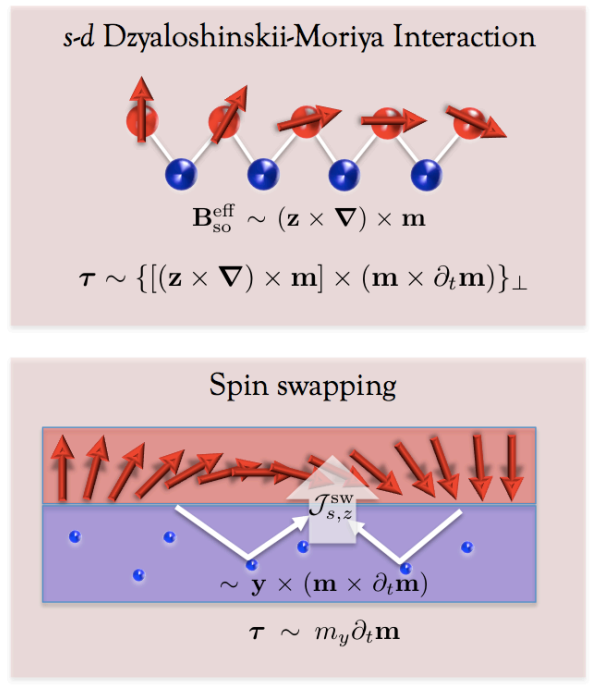

Figure 28: (Color online) Illustration of different microscopic mechanisms at the origin of chiral damping: (a) the interplay of inverse spin galvanic effect (ISGE) and spin transfer torque (STT) in the presence of Rashba spin-orbit coupling, (b) $s$-d Dzyaloshinskii-Moriya interaction, (c) spin pumping in the presence of anomalous Hall effect (AHE) and (d) the interplay between spin pumping and spin swapping. All these mechanisms give rise to a magnetic damping that is linear in spin-orbit coupling and magnetization gradient.

not contribute to additional electric signal, it produces an anisotropic damping on the form $\boldsymbol{\tau} \sim m_{y} \partial_{t} \mathbf{m}$. This effect vanishes by symmetry in homogeneous ferromagnets, but is expected to survive in magnetic domain walls resulting in unconventional magnetic damping.

\subsection{Topological Torques}

As discussed above, the presence of spin-orbit coupling at transition metal interfaces promotes the emergence of chiral textures, such as homochiral Néel walls $[69,262,263,50,51,52]$ that display very large velocities [264, 11, 262, 12] or magnetic skyrmions $[13,47,45,44,14,265]$. The latter are topological magnetic defects [36] that present some similarities with the more traditional magnetic vortices [266, 267, 268, 269]. In both cases, the magnetic topology induces a Lorentz force on the flowing electrons, resulting in topological Hall effect $[270,36]$. Both classes of magnetic textures also experience a Hall effect when driven by an electric flow, an effect sometimes referred to as skyrmion Hall effect, and recently observed in transition metal interfaces [15, 16].

The reason why magnetic skyrmions are currently attracting a massive amount of interest is because simulations suggest that they get very weakly pinned on defects, enabling motion at low critical current density and long data retention $[271,272,273,274]$. That being said, the very recent observation of currentdriven motion of skyrmions at transition metal interfaces suggests on contrary that in polycrystalline samples the motion is quite sensitive to disorder and 
grain boundaries $[13,14,15,16,275]$. To learn how to control the motion of such chiral textures, one needs to develop a precise understanding of the nature of the torque exerted on these objects. In magnetic textures, the spin transfer torque can be generally expressed as

$$
\boldsymbol{\tau}=b_{\mathrm{J}}(\mathbf{u} \cdot \nabla) \mathbf{m}-\beta b_{\mathrm{J}} \mathbf{m} \times(\mathbf{u} \cdot \nabla) \mathbf{m},
$$

where $\mathbf{u}$ is the direction of current injection, $\mathbf{m}$ is a unit vector in the direction of the magnetization. The two terms on the right hand side of Eq. (48) are called the adiabatic $\left(\sim b_{\mathrm{J}}\right)$ and non-adiabatic torques $\left(\sim \beta b_{\mathrm{J}}\right)$, respectively $[276,277]$. While the non-adiabatic parameter $\beta$ in transverse walls is quite small $\beta \approx \alpha$ ( $\alpha$ being the magnetic damping of the homogeneous magnet), it is much larger in magnetic vortices and reaches $8 \alpha$ to $10 \alpha$ [267, 268, 269, 278]. Several contributions to the non-adiabatic parameter have been identified: spin relaxation [276] $\left(\beta \sim \lambda_{\mathrm{ex}}^{2} / \lambda_{\mathrm{sf}}^{2}\right)$, spin mistracking [279, 280, 281] $\left(\beta \sim e^{-\lambda_{\mathrm{dw}} / \lambda_{\mathrm{ex}}}\right)$, anomalous Hall effect [282] $\left(\beta \sim \theta_{\mathrm{H}}\right)$, and more recently spin diffusion [283] $\left(\beta \sim \lambda_{\mathrm{ex}}^{2} / \lambda_{\mathrm{dw}}^{2}\right)$. Here $\lambda_{\mathrm{dw}}$ is the domain wall width, $\lambda_{\mathrm{ex}, \mathrm{sf}}$ are the transverse and longitudinal spin relaxation lengths and $\theta_{\mathrm{H}}$ is the anomalous Hall angle.

More recently, a few authors proposed that topological Hall effect, inherent to both magnetic vortices and skyrmions, can lead to enhanced non-adiabaticity $[278,284,271,285]$. We believe this "topological torque" is of great importance for current-driven skyrmion motion because it increases when reducing the size of the skyrmion, and can therefore lead to improved dynamical properties upon size reduction, as discussed at the end of this Section.

\subsubsection{Emergent Electrodynamics}

When conduction electrons flow in a smooth and slow magnetic texture, $\mathbf{m}(\mathbf{r}, t)$, their spin adiabatically changes orientation so that these electrons acquire a Berry phase [286, 287, 288]. This geometrical phase can be represented by an emergent electromagnetic field $\left(\mathbf{E}_{\mathrm{em}}, \mathbf{B}_{\mathrm{em}}\right)$ determined by the magnetic texture gradients $[289,290,253,254,280,255,291]$. A useful way to see that is to consider the simplest two dimensional $s$ - $d$ Hamiltonian (extension to three dimensions is straightforward)

$$
\hat{\mathcal{H}}=\frac{\hat{\mathbf{p}}^{2}}{2 m}+J_{\mathrm{sd}} \hat{\boldsymbol{\sigma}} \cdot \mathbf{m}(\mathbf{r}, t) .
$$

The Schrödinger equation corresponding to Eq. (49) can be re-written in the rotating frame of the magnetization, using the unitary transformation $\mathcal{U}=$ $e^{-i \frac{\theta}{2} \hat{\boldsymbol{\sigma}} \cdot \mathbf{n}}$ where $\mathbf{n}=\mathbf{z} \times \mathbf{m} /|\mathbf{z} \times \mathbf{m}|$ to obtain

$$
\tilde{\mathcal{H}}=\frac{(\hat{\mathbf{p}}-e \hat{\mathcal{A}})^{2}}{2 m}+J_{\mathrm{sd}} \hat{\sigma}_{z}+e \hat{\mathcal{V}}
$$

where the vector and scalar potentials are given respectively as $\hat{\mathcal{A}}=-\frac{\hbar}{2 e} \hat{\boldsymbol{\sigma}}$. $\left(\mathbf{m} \times \partial_{i} \mathbf{m}\right) \mathbf{e}_{i}$ and $\hat{\mathcal{V}}=\frac{\hbar}{2 e} \hat{\boldsymbol{\sigma}} \cdot\left(\mathbf{m} \times \partial_{t} \mathbf{m}\right)$. Hence, the electromagnetic field can be 
written the form $[292,290,253,254,280,255,291,293,294,257,295]$

$$
\begin{aligned}
& \mathbf{E}_{\mathrm{em}}^{s}=-\left\langle s\left|\partial_{i} \hat{\mathcal{V}}+\partial_{t} \hat{\mathcal{A}}_{i}\right| s\right\rangle=\frac{s \hbar}{2 e}\left[\mathbf{m} \cdot\left(\partial_{t} \mathbf{m} \times \partial_{i} \mathbf{m}\right)\right] \mathbf{e}_{i}, \\
& \mathbf{B}_{\mathrm{em}}^{s}=\langle s|\nabla \times \hat{\mathcal{A}}| s\rangle=-\frac{s \hbar}{2 e}\left[\mathbf{m} \cdot\left(\partial_{x} \mathbf{m} \times \partial_{y} \mathbf{m}\right)\right] \mathbf{z} .
\end{aligned}
$$

Here, $|s\rangle$ denotes the eigenstate of Eq. (50) in the absence of emergent electromagnetic field. As a result, one can compute the charge current for spin $s$ [278]

$$
\mathbf{j}_{e}^{s}=\sigma_{0}^{s} \mathbf{E}+\sigma_{0}^{s} \mathbf{E}_{\mathrm{em}}^{s}+\frac{\sigma_{H}^{s}}{B_{\mathrm{H}}} \mathbf{E} \times \mathbf{B}_{\mathrm{em}}^{s}+\frac{\sigma_{H}^{s}}{B_{\mathrm{H}}} \mathbf{E}_{\mathrm{em}}^{s} \times \mathbf{B}_{\mathrm{em}}^{s},
$$

where $\sigma_{0}^{s}$ and $\sigma_{H}^{s}$ are respectively the longitudinal and ordinary Hall conductivities for spin $s, \mathbf{E}=E \mathbf{u}$ is the external electric field, and $B_{\mathrm{H}}=\left|\mathbf{B}_{\mathrm{em}}^{s}\right|$. From Eq. (53), we note that the spin-dependent current is driven by the external electric field $\mathbf{E}$ as well as by the emergent electric field governed by the time-variation of the magnetic texture, $\mathbf{E}_{\mathrm{em}}^{s}$. We can obtain the expressions for the local spin current tensor, $\mathcal{J}_{s}=-\mathbf{m} \otimes\left(\mathbf{j}_{e}^{\uparrow}-\mathbf{j}_{e}^{\downarrow}\right)$, and charge current vector, $\mathbf{j}_{e}=\mathbf{j}_{e}^{\uparrow}+\mathbf{j}_{e}^{\downarrow}$, from Eq. (53). For a static texture $\left(\partial_{t} \mathbf{m}=0\right)$, we obtain

$$
\begin{aligned}
\mathcal{J}_{s} & =-b_{\mathrm{J}} \mathbf{m} \otimes \mathbf{u}+\frac{\mathcal{P}_{H}}{\mathcal{P}_{0}} \lambda_{H}^{2} b_{\mathrm{J}}\left[\mathbf{m} \cdot\left(\partial_{x} \mathbf{m} \times \partial_{y} \mathbf{m}\right)\right] \mathbf{m} \otimes \mathbf{z} \times \mathbf{u}, \\
\mathbf{j}_{e} & =\sigma_{0} E \mathbf{u}-\sigma_{0} \lambda_{H}^{2} E\left[\mathbf{m} \cdot\left(\partial_{x} \mathbf{m} \times \partial_{y} \mathbf{m}\right)\right] \mathbf{z} \times \mathbf{u},
\end{aligned}
$$

where $\sigma_{a}=\sigma_{a}^{\uparrow}+\sigma_{a}^{\downarrow}, \mathcal{P}_{a}=\left(\sigma_{a}^{\uparrow}-\sigma_{a}^{\downarrow}\right) / \sigma_{a},(a=0, \mathrm{H}), \lambda_{\mathrm{H}}^{2}=\hbar \sigma_{H} / 2 e \sigma_{0} B_{\mathrm{H}}$. In addition to the usual constant spin current moving along the direction of the applied electric field $(\sim \mathbf{u})$, the presence of a non-zero topological charge, $\mathbf{m} \cdot\left(\partial_{x} \mathbf{m} \times \partial_{y} \mathbf{m}\right) \neq 0$ (as it is the case for magnetic skyrmions and vortices), leads to a texture-induced emergent magnetic field that induces an additional spatially varying spin current along both the longitudinal $(\sim \mathbf{u})$ and transverse $(\sim \mathbf{z} \times \mathbf{u})$ directions to the electric field $\mathbf{E}$. This longitudinal spin current is responsible for (i) topological spin and charge Hall effects already observed in topological textures such as skyrmions [290, 270, 36, 296] and, (ii) enhanced non-adiabaticity already observed in vortices [278], as illustrated on Fig. 29.

\subsubsection{Topological spin torque}

Through spin transfer mechanism, the spin current given in Eq. (54) exerts a torque on the local magnetization, $\boldsymbol{\tau}=-\boldsymbol{\nabla} \cdot \mathcal{J}_{s}$, which explicitly reads

$$
\boldsymbol{\tau}=b_{J}(\mathbf{u} \cdot \boldsymbol{\nabla}) \mathbf{m}-\frac{\mathcal{P}_{\mathrm{H}}}{\mathcal{P}_{0}} \lambda_{\mathrm{H}}^{2} b_{J}\left[\mathbf{m} \cdot\left(\partial_{x} \mathbf{m} \times \partial_{y} \mathbf{m}\right)\right][(\mathbf{z} \times \mathbf{u}) \cdot \boldsymbol{\nabla}] \mathbf{m} .
$$

A remarkable consequence of Eq. (56) is that since the second term is even upon magnetization reversal, it induces an intrinsic topological non-adiabatic spin transfer torque. This non-adiabatic torque is intrinsic as it does not rely on impurities or defects (in contrast with the non-adiabaticity studied in Refs. $[276,283])$, and topological since its origin is associated to the topology of the magnetic texture. 


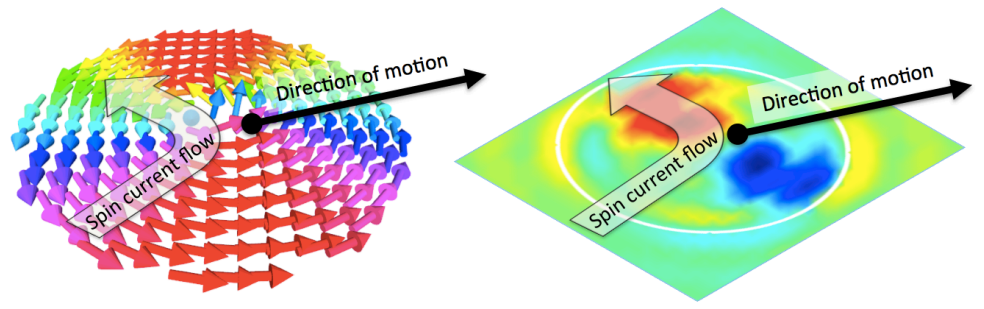

Figure 29: (Color online) The non-trivial topological texture of magnetic vortices and skyrmions distorts the trajectory of flowing electrons (left panel). This effect, called the topological Hall effect, can be clearly seen from tight-binding calculations (right panel), where the spin accumulation in the center of the magnetic vortex displays an antisymmetric distribution that is characteristic of spin Hall effect, although no spin-orbit coupling is present [278]. This asymmetric spin accumulation is a direct consequence of topological Hall effect and exerts a torque on the local texture, thereby inducing the so-called skyrmion Hall effect.

To quantify the topological torque, we first study the current-driven dynamics of an isolated magnetic skyrmion and vortex. To do so, we adopt the rigid motion formalized developed by Thiele [297]. The equation of motion governing the dynamics of these structures is given by the extended LLG equation

$$
\partial_{t} \mathbf{m}=-\gamma \mathbf{m} \times \mathbf{H}_{\mathrm{eff}}+\alpha \mathbf{m} \times \partial_{t} \mathbf{m}+\boldsymbol{\tau}-\beta b_{\mathrm{J}} \mathbf{m} \times \partial_{x} \mathbf{m}
$$

where $\beta$ is a constant non-adiabatic parameter introduced by hand [276, 277]. The magnetization profile in spherical coordinates is given by the polar angle $\theta$ and the azimuthal angle $\varphi$. The polar angle is defined for an isolated skyrmion as $\cos \theta=p\left(r_{0}^{2}-r^{2}\right) /\left(r_{0}^{2}+r^{2}\right)$, and for an isolated vortex as $\cos \theta=p\left(r_{0}^{2}-\right.$ $\left.r^{2}\right) /\left(r_{0}^{2}+r^{2}\right)$ for $r \leq r_{0}$, and $\theta=\pi / 2$ for $r>r_{0} \cdot p= \pm 1$ defines the skyrmion (vortex core) polarity, $r_{0}$ is the radius of the skyrmion (vortex core). For both textures, the azimuthal angle is defined $\Phi=q \operatorname{Arg}(x+i y)+c \pi / 2$, where $q= \pm 1$ is the vorticity and the $c= \pm 1$ defines the chirality. Using these profiles, we obtain the analytical expressions of the velocity components as [284]

$$
\begin{aligned}
& v_{x}=-\frac{1+\alpha_{\mathrm{eff}} \beta_{\mathrm{eff}}}{1+\alpha_{\mathrm{eff}}^{2}} b_{J}, \\
& v_{y}=p q \frac{\beta_{\mathrm{eff}}-\alpha_{\mathrm{eff}}}{1+\alpha_{\mathrm{eff}}^{2}} b_{J},
\end{aligned}
$$

where $\mathcal{C}=1+\ln \sqrt{R / r_{0}}$, and $\beta_{\text {eff }}$ and $\alpha_{\text {eff }}$ are the effective non-adiabatic parameter and enhanced magnetic damping, respectively. In the present discussion, we neglect the effect of the enhanced damping, treated in Ref. [284], and focus on the topological torque. The effective non-adiabatic parameter read,

$$
\beta_{\text {eff }}= \begin{cases}\beta+\frac{4 \mathcal{S}_{2}}{3} \frac{\mathcal{P}_{\mathrm{H}}}{\mathcal{P}_{0}} \frac{\lambda_{\mathrm{H}}^{2}}{r_{0}^{2}}, & \text { for skyrmion } \\ \beta \mathcal{C}+\frac{7}{3} \frac{\mathcal{P}_{\mathrm{H}}}{\mathcal{P}_{0}} \frac{\lambda_{\mathrm{H}}^{2}}{r_{0}^{2}}, & \text { for vortex core }\end{cases}
$$


where $\mathcal{S}=r_{0}^{2} /\left(r_{0}^{2}+R^{2}\right)$, and $\mathcal{S}_{k}=\sum_{i=0}^{i=k}(\mathcal{S})^{i}$. Remarkably, in both cases the topological non-adiabaticity scales with $1 / r_{0}^{2}$. In other words, the sharper the texture, the larger the non-adiabatic torque and the larger the skyrmion Hall effect. Reducing the skyrmion (or vortex core) size by a factor of 2 enhances the non-adiabaticity by a factor of 4 . Using realistic material parameters $r_{0}=30 \mathrm{~nm}, \theta_{\mathrm{TH}}=\sigma_{H} / \sigma_{0}=0.01$, and $B_{H}=2.5 \mathrm{~T}$ [298], we obtain $\lambda_{H}^{2}=1.32 \mathrm{~nm}^{2}$ and the corresponding topological contribution to the nonadiabaticity parameter $\beta=0.08$.

\subsubsection{Tight-binding model}

The analytical derivation proposed above relies on the assumption that spin transport is adiabatic throughout the magnetic texture, i.e. the spin of the conduction electrons remains aligned on the local magnetization direction and no spin mistracking is considered [279]. To confirm the analytical results, we computed the topological transport and torques in a magnetic skyrmions numerically using the non-equilibrium wave function formalism implemented on a tight-binding model in the KWANT package [205]. The system is composed of a scattering region containing an isolated skyrmion or vortex core, attached to two ferromagnetic leads. In the same spirit as the tight-binding models previously discussed, the system is a two dimensional square lattice described by the Hamiltonian [296, 284]

$$
\hat{\mathcal{H}}=\sum_{i}\left(\epsilon_{i} \hat{c}_{i}^{\dagger} \hat{c}_{i}+\Delta \hat{c}_{i}^{\dagger} \hat{\boldsymbol{\sigma}} \cdot \mathbf{m}_{i} \hat{c}_{i}\right)-t \sum_{<i j>} \hat{c}_{i}^{\dagger} \hat{c}_{j},
$$

where the sum $\langle i j\rangle$ is restricted to nearest neighbors, and the skyrmion texture is encoded in the unit vector $\mathbf{m}_{i}$. The torque $\boldsymbol{\tau}_{n}$ at site $\mathbf{n}$ is computed by using the local spin densities as

$$
\boldsymbol{\tau}_{n}=\frac{2 \Delta}{\hbar} \mathbf{m}_{n} \times\left\langle\mathbf{S}_{n}\right\rangle=\tau_{n}^{\mathrm{ad}} \partial_{x} \mathbf{m}_{n}-\tau_{n}^{\mathrm{na}} \mathbf{m}_{n} \times \partial_{x} \mathbf{m}_{n},
$$

To investigate the total torque exerted on the magnetic skyrmion, we calculated the normalized adiabatic and non-adiabatic torque components by integrating the projections of the local torque on $\mathbf{m} \times \partial_{y} \mathbf{m}$ and $\mathbf{m} \times \partial_{x} \mathbf{m}$ respectively and normalized accordingly i.e.

$$
\begin{aligned}
\tilde{\tau}_{\mathrm{ad}} & =\frac{\int \boldsymbol{\tau} \cdot\left(\mathbf{m} \times \partial_{y} \mathbf{m}\right) d^{2} \mathbf{r}}{\int \mathbf{m} \cdot\left(\partial_{x} \mathbf{m} \times \partial_{y} \mathbf{m}\right) d^{2} \mathbf{r}}, \\
\tilde{\tau}_{\text {na }} & =\frac{\int \boldsymbol{\tau} \cdot\left(\mathbf{m} \times \partial_{x} \mathbf{m}\right) d^{2} \mathbf{r}}{\int \mathbf{m} \cdot\left(\partial_{x} \mathbf{m} \times \partial_{y} \mathbf{m}\right) d^{2} \mathbf{r}} .
\end{aligned}
$$

Figure 30 displays both torques as a function of the skyrmion radius $r_{0}$. While the adiabatic torque (open symbols) is almost constant, the non-adiabatic torque (filled symbols) shows a substantial dependence on the skyrmion radius $r_{0}$, in agreement with the analytical results presented in Eq. (60). Besides providing a reasonable explanation for the enhanced non-adiabaticity in skyrmions and 


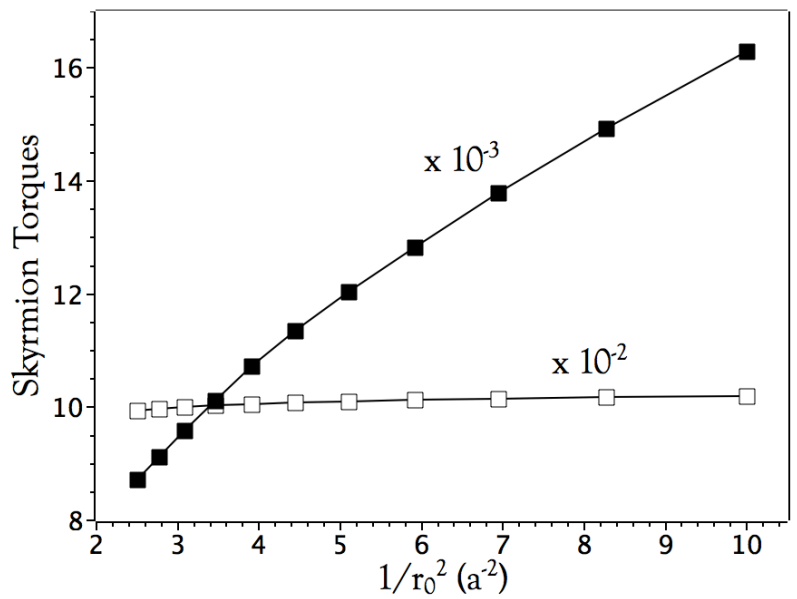

Figure 30: (Color online) Effective adiabatic and non-adiabatic torque dependence on the skyrmion radius. The adiabatic torque (open symbols) is almost non-dependent on the radius of the skyrmion while the non-adiabatic torque (filled symbols) shows substantial dependence on the skyrmion radius $r_{0}$. From [284].

magnetic vortices [278], this topological torque opens interesting perspectives for the investigation of current-driven skyrmion dynamics. Since the topological torque is an intrinsic property of the magnetic texture, one could continuously deform this structure by applying, e.g., a perpendicular external magnetic field $[299,300]$, and therefore influence the non-adiabaticity parameter. For instance, Moreau-Luchaire et. al. [44] demonstrated a reduction of the skyrmion diameter from $80 \mathrm{~nm}$ at $12 \mathrm{mT}$ to $30 \mathrm{~nm}$ at $70 \mathrm{mT}$. Following our predictions, the topological non-adiabaticity parameter should increase by a factor $\sim 7$ in this specific case. 


\section{Conclusion}

The simultaneous presence of spin-orbit coupling, magnetism and interfacial symmetry breaking offers a vast playground for spintronics and modern magnetism, enabling the efficient electrical control of exotic magnetic textures. Although most of the physics at stake can be accounted for through phenomenological models (free electron model, Rashba spin-orbit coupling, adiabatic spin transport, micromagnetics etc.), the recent systematic investigation of spin-orbit coupled transition metal interfaces using first principles methods has nurtured major progress in the field. For instance, spin Hall effect [151], spin-orbit splitting [22] and DMI [21] have been investigated for a wide range of $3 d / 5 d(4 d)$ interfaces and systematic trends, well understood in terms of $d$-orbital hybridizations, have emerged. Yet, there are still several significant challenges that remain to be tackled. Offering a coherent description of the interplay between inverse spin galvanic effect, interfacial and bulk spin Hall effects at these interfaces is highly desirable to foster the realization of efficient spin-orbit torque devices. The concept of chiral damping, recently introduced [301, 260] needs to be rigorously treated using both linear response theory and ab initio approaches. The correlation between all these effects and their dependence as a function of the nature of the orbital hybridization remain to be thoroughly understood.

Besides ferromagnetic interfaces, two large classes of systems are currently drawing an increasing amount of attention. The first class concerns the interfaces between heavy metals and antiferromagnets [302, 303]. As a matter of fact, the prediction of spin transfer torque in antiferromagnetic spin-valves ten years ago [304] has opened thrilling perspectives in the field of spintronics and beyond. These materials possess thought-provoking dynamical behavior, and, most importantly, can be manipulated electrically using spin-orbit torques [305, 306, 307, 308]. Spin Hall effect has been the object of a recent study [182], but the field is still at its infancy and all the effects discussed in the present chapter, such as antiferromagnetic skyrmions [309, 310], should also emerge in antiferromagnetic multilayers.

The second class of materials that could entirely renew this already fascinating field concerns the so-called topological materials, i.e. materials whose band structures are topologically non-trivial. We already briefly mentioned the topological insulators [311, 312, 313], which possess spin-orbit coupled surface states and remained to be understood in the context of spin-orbit torques. Other materials such as crystalline [314], Anderson [315, 316] or Kondo topological insulators [317], but also Weyl semimetals [318, 319] and Dirac semimetals [320] all possess very large spin-orbit coupling and display exotic surface states. For instance, Weyl semimetals are characterized by their surface Fermi arcs, i.e. disrupted Fermi surfaces that connect two opposite surfaces through bulk states [318]. In this ever-growing class of materials, spin-charge conversion processes (spin Hall, spin swapping, inverse spin galvanic effects) are yet to be explored theoretically and experimentally and the interplay between electric currents and magnetism holds promises for even more thrilling adventures. 


\section{Acknowledgments}

The authors are grateful for the inspiring discussions with S. Blügel, G.

${ }_{1636}$ Bihlmayer, P.M. Haney, S. Grytsyuk, G. Gaudin, M. Miron, V. Baltz, M. Kläui,

${ }_{1637}$ H. Yang, J. Sinova, T. Jungwirth, X. Waintal, M. Chshiev, X.X. Zhang, J.

1638 Kosel, and B. Nikolić. This work is supported by the King Abdullah University 1639 of Science and Technology (KAUST). 
[1] M. Johnson, P. J. H. Bloemenz, F. J. A. den Broeder, J. J. de Vries, Magnetic anisotropy in metallic multilayers, Rep. Prog. Phys. 59 (1996) 1409 . URL http://iopscience.iop.org/0034-4885/59/11/002

[2] M. Wu, J. Jiang, M. Weng, Spin dynamics in semiconductors, Physics Reports 493 (2-4) (2010) 61-236. doi:10.1016/j.physrep.2010.04.002. URL http://linkinghub.elsevier.com/retrieve/pii/ S0370157310000955

[3] D. L. Mills, S. M. Rezende, Spin Damping in Ultrathin Magnetic Films, Topics App. Phys. 87 (2003) 27-59.

[4] T. McGuire, R. Potter, Anisotropic magnetoresistance in ferromagnetic 3d alloys, IEEE Transactions on Magnetics 11 (1975) 1018-1038.

[5] N. Nagaosa, J. Sinova, S. Onoda, A. H. MacDonald, N. P. Ong, Anomalous Hall effect, Rev. Mod. Phys. 82 (2) (2010) 1539-1592. doi:10.1103/ RevModPhys.82.1539.

URL http://link.aps.org/doi/10.1103/RevModPhys.82.1539

[6] A. Manchon, H. C. Koo, J. Nitta, S. M. Frolov, R. A. Duine, New perspectives for Rashba spin orbit coupling, Nature Materials 14 (9) (2015) 871-882. doi:10.1038/nmat4360.

URL http://dx.doi.org/10.1038/nmat4360

[7] A. Soumyanarayanan, N. Reyren, A. Fert, C. Panagopoulos, Spin-Orbit Coupling Induced Emergent Phenomena at Surfaces and Interfaces, Nature 539 (7630) (2016) 509-517. arXiv:1611.09521, doi:10.1038/ nature19820. URL http://dx.doi.org/10.1038/nature19820

[8] I. M. Miron, K. Garello, G. Gaudin, P.-J. Zermatten, M. V. Costache, S. Auffret, S. Bandiera, B. Rodmacq, A. Schuhl, P. Gambardella, Perpendicular switching of a single ferromagnetic layer induced by in-plane current injection., Nature 476 (7359) (2011) 189-93. doi:10.1038/ nature10309. URL http://www.ncbi.nlm.nih.gov/pubmed/21804568

[9] L. Liu, C.-F. Pai, Y. Li, H. W. Tseng, D. C. Ralph, R. A. Buhrman, Spin-torque switching with the giant spin Hall effect of tantalum., Science 336 (6081) (2012) 555-558. doi:10.1126/science.1218197.

URL http://www.ncbi.nlm.nih.gov/pubmed/22556245

[10] K. Garello, C. O. Avci, I. M. Miron, M. Baumgartner, A. Ghosh, S. Auffret, O. Boulle, G. Gaudin, P. Gambardella, Ultrafast magnetization switching by spin-orbit torques, Applied Physics Letters 105 (21) (2014) 212402. doi:10.1063/1.4902443.

URL http://scitation.aip.org/content/aip/journal/apl/105/21/ 10.1063/1.4902443 
[11] I. M. Miron, T. Moore, H. Szambolics, L. D. Buda-Prejbeanu, S. Auffret, B. Rodmacq, S. Pizzini, J. Vogel, M. Bonfim, A. Schuhl, G. Gaudin, Fast current-induced domain-wall motion controlled by the Rashba effect., Nature Materials 10 (6) (2011) 419-23. doi:10.1038/nmat3020.

URL http://www.ncbi.nlm.nih.gov/pubmed/21572411

[12] S.-H. Yang, K.-S. Ryu, S. Parkin, Domain-wall velocities of up to 750 $\mathrm{m}$ s1 driven by exchange-coupling torque in synthetic antiferromagnets, Nature Nanotechnology 10 (February) (2015) 221-226. doi:10.1038/ nnano. 2014.324.

URL http://www .nature.com/doifinder/10.1038/nnano.2014.324

[13] W. Jiang, P. Upadhyaya, W. Zhang, G. Yu, M. B. Jungfleisch, F. Y. Fradin, J. E. Pearson, Y. Tserkovnyak, K. L. Wang, O. Heinonen, S. G. E. Velthuis, A. Hoffmann, Blowing magnetic skyrmion bubbles, Science 349 (2015) 283.

[14] S. Woo, K. Litzius, B. Krüger, M.-Y. Im, L. Caretta, K. Richter, M. Mann, A. Krone, R. M. Reeve, M. Weigand, P. Agrawal, I. Lemesh, M.-A. Mawass, P. Fischer, M. Kläui, G. S. D. Beach, Observation of roomtemperature magnetic skyrmions and their current-driven dynamics in ultrathin metallic ferromagnets, Nature Materials 15 (5) (2016) 501-506. arXiv:1502.07376, doi:10.1038/nmat4593. URL http://arxiv.org/abs/1502.07376\{\%\}5Cnhttp://www nature. com/doifinder/10.1038/nmat4593

[15] W. Jiang, X. Zhang, G. Yu, W. Zhang, M. B. Jungfleisch, J. E. Pearson, O. Heinonen, K. L. Wang, Y. Zhou, A. Hoffmann, S. G. E. te Velthuis, Direct Observation of the Skyrmion Hall Effect, Nature Physics 13 (2017) 162. arXiv: 1603.07393, doi:10.1038/nphys3883. URL http://arxiv.org/abs/1603.07393

[16] K. Litzius, I. Lemesh, B. Krüger, L. Caretta, K. Richter, F. Büttner, P. Bassirian, J. Förster, R. M. Reeve, M. Weigand, I. Bykova, H. Stoll, G. Schütz, G. S. D. Beach, M. Kläui, Skyrmion Hall Effect Revealed by Direct Time-Resolved X-Ray Microscopy, Nature Physics 13 (2017) 170. arXiv:1608.07216, doi:10.1038/nphys4000.

URL http://arxiv.org/abs/1608.07216

[17] D. A. Papaconstantopoulos, Handbook of the Band Structure of Elemental Solids, second edi Edition, Springer, New York, 2015. doi:10.1016/ 0001-6160 (66) 90168-4.

[18] S. Blügel, G. Bihlmayer, Magnetism of Low-dimensional Systems : Theory, in: H. Kronmuller, S. Parkin (Eds.), Handbook of Magnetism and Advanced Magnetic Materials., Vol. 1, John Wiley \& Sons, 2007, pp. 142. doi: 10.1002/9780470022184.

URL http://doi.wiley.com/10.1002/9780470022184 
[19] S. Blugel, M. Weinert, P. Dederichs, Ferromagnetism and Antiferromagnetism of 3d-Metal Overlayers on Metals, Phys. Rev. Lett. 60 (11) (1988) 1077-1080.

[20] S. Blugel, Two-dimensional ferromagnetism of 3d, 4d, and $5 \mathrm{~d}$ transition metal monolayers on noble metal (001) substrates, Physical Review Letters 68 (6) (1992) 851-854. doi:10.1103/PhysRevLett.68.851.

[21] A. Belabbes, G. Bihlmayer, F. Bechstedt, S. Blügel, A. Manchon, Hund's rule-driven Dzyaloshinskii-Moriya interaction at 3d-5d interfaces, Phys. Rev. Lett. 117 (December) (2016) 247202. arXiv:1609.04004, doi:10. 1103/PhysRevLett.117.247202. URL http://arxiv.org/abs/1609.04004

[22] S. Grytsyuk, A. Belabbes, P. M. Haney, H. W. Lee, K. J. Lee, M. D. Stiles, U. Schwingenschlögl, A. Manchon, K -asymmetric spin splitting at the interface between transition metal ferromagnets and heavy metals, Physical Review B - Condensed Matter and Materials Physics 93 (17) (2016) 174421. doi:10.1103/PhysRevB.93.174421.

[23] N. Nakajima, T. Koide, T. Shidara, H. Miyauchi, H. Fukutani, A. Fujimori, K. Iio, T. Katayama, M. Nývlt, Y. Suzuki, Perpendicular Magnetic Anisotropy Caused by Interfacial Hybridization via Enhanced Orbital Moment in Co Pt Multilayers : Magnetic Circular X-Ray Dichroism Study, Phys. Rev. Lett. 81 (23) (1998) 5229-5232.

[24] O. Sipr, S. Bornemann, H. Ebert, S. Mankovsky, J. Vackar, J. Minar, Co monolayers and adatoms on $\operatorname{Pd}(100), \operatorname{Pd}(111)$, and $\operatorname{Pd}(110)$ : Anisotropy of magnetic properties, Physical Review B - Condensed Matter and Materials Physics 88 (6) (2013) 064411. arXiv:1303.5262, doi:10.1103/PhysRevB.88.064411.

[25] A. Lehnert, S. Dennler, P. Blonski, S. Rusponi, M. Etzkorn, G. Moulas, P. Bencok, P. Gambardella, H. Brune, J. Hafner, Magnetic anisotropy of $\mathrm{Fe}$ and Co ultrathin films deposited on $\mathrm{Rh}(111)$ and $\mathrm{Pt}(111)$ substrates: An experimental and first-principles investigation, Physical Review B Condensed Matter and Materials Physics 82 (9) (2010) 094409. doi: 10.1103/PhysRevB.82.094409.

[26] S. Bornemann, O. Aipr, S. Mankovsky, S. Polesya, J. B. Staunton, W. Wurth, H. Ebert, J. Minar, Trends in the magnetic properties of Fe, $\mathrm{Co}$, and Ni clusters and monolayers on $\operatorname{Ir}(111), \mathrm{Pt}(111)$, and $\mathrm{Au}(111)$, Physical Review B 86 (10) (2012) 1-12. arXiv:arXiv:1204.6230v1, doi:10.1103/PhysRevB.86.104436.

[27] G. Dresselhaus, Spin-Orbit Coupling Effects in Zinc Blende Structures, Phys. Rev. 100 (2) (1955) 580. 
[28] F.T. Vasko, Spin splitting in the spectrum of two-dimensional electrons due to the surface potential, Pis'ma Zh. Eksp. Teor. Fiz 30 (9) (1979) 574.

[29] Y. a. Bychkov, E. I. Rashba, Oscillatory effects and the magnetic susceptibility of carriers in inversion layers, Journal of Physics C: Solid State Physics 17 (33) (1984) 6039-6045. doi:10.1088/0022-3719/17/33/015. URL http://stacks.iop.org/0022-3719/17/i=33/a=015?key= crossref.2f8159f54a3070499f32bac53e23f 947

[30] P. M. Haney, H. W. Lee, K. J. Lee, A. Manchon, M. D. Stiles, Currentinduced torques and interfacial spin-orbit coupling, Physical Review B Condensed Matter and Materials Physics 88 (21) (2013) 214417. arXiv: arXiv:1309.1356v1.

[31] G. Bihlmayer, Y. Koroteev, P. Echenique, E. Chulkov, S. Blügel, The Rashba-effect at metallic surfaces, Surface Science 600 (18) (2006) 3888-3891. doi:10.1016/j.susc.2006.01.098.

URL http://linkinghub.elsevier.com/retrieve/pii/ S0039602806004195

[32] G. Bihlmayer, S. Blügel, E. Chulkov, Enhanced Rashba spin-orbit splitting in $\operatorname{BiAg}(111)$ and $\operatorname{PbAg}(111)$ surface alloys from first principles, Phys. Rev. B 75 (19) (2007) 195414. doi:10.1103/PhysRevB.75.195414. URL http://link.aps.org/doi/10.1103/PhysRevB.75.195414

[33] M. Nagano, A. Kodama, T. Shishidou, T. Oguchi, A first-principles study on the Rashba effect in surface systems., Journal of physics. Condensed matter : an Institute of Physics journal 21 (6) (2009) 064239. doi:10. 1088/0953-8984/21/6/064239. URL http://www.ncbi.nlm.nih.gov/pubmed/21715941

[34] H. Bentmann, T. Kuzumaki, G. Bihlmayer, S. Blügel, E. V. Chulkov, F. Reinert, K. Sakamoto, Spin orientation and sign of the Rashba splitting in Bi/Cu(111), Phys. Rev. B 84 (11) (2011) 115426. doi:10.1103/ PhysRevB.84.115426.

URL http://link.aps.org/doi/10.1103/PhysRevB.84.115426

[35] I. V. Tokatly, E. E. Krasovskii, G. Vignale, Current-induced spin polarization at the surface of metallic films: A theorem and an ab initio calculation, Physical Review B - Condensed Matter and Materials Physics 91 (3) (2015) 035403. arXiv:1410.3693, doi:10.1103/PhysRevB.91.035403.

[36] N. Nagaosa, Y. Tokura, Topological properties and dynamics of magnetic skyrmions., Nature nanotechnology 8 (12) (2013) 899-911. doi:10.1038/ nnano.2013.243.

URL http://www.ncbi.nlm.nih.gov/pubmed/24302027

[37] I. Dzyaloshinskii, Thermodynamic theory of weak ferromagnetism in antiferromagnetic substances, Sov. Phys. JETP 5 (1957) 1259. 
[38] T. Moriya, Anisotropic superexchange interaction and weak ferromagnetism, Phys. Rev. 120 (1) (1960) 91-98. doi:http: //dx.doi.org/10.1103/PhysRev.120.91. URL https://journals.aps.org/pr/pdf/10.1103/PhysRev.120. 91http://prola.aps.org/abstract/PR/v120/i1/p91\{_\}1

[39] A. Bogdanov, U. Rößler, Chiral Symmetry Breaking in Magnetic Thin Films and Multilayers, Phys. Rev. Lett. 87 (3) (2001) 037203. doi:10. 1103/PhysRevLett.87.037203. URL http://link.aps.org/doi/10.1103/PhysRevLett.87.037203

[40] U. K. Roessler, A. N. Bogdanov, C. Pfleiderer, Spontaneous skyrmion ground states in magnetic metals, Nature 442 (7104) (2006) 797-801. URL http://dx.doi.org/10.1038/nature05056

[41] S. Mühlbauer, B. Binz, F. Jonietz, C. Pfleiderer, Skyrmion lattice in a chiral magnet, Science (New York, N.Y.) 323 (September) (2009) 915920.

URL http://www . sciencemag. org/content/323/5916/915. short

[42] X. Z. Yu, Y. Onose, N. Kanazawa, J. H. Park, J. H. Han, Y. Matsui, N. Nagaosa, Y. Tokura, Real-space observation of a two-dimensional skyrmion crystal, Nature 465 (7300) (2010) 901-904.

URL http://dx.doi.org/10.1038/nature09124http://www. nature. com/nature/journal/v465/n7300/suppinfo/nature09124\{_\}S1.html

[43] S. Heinze, K. von Bergmann, M. Menzel, J. Brede, A. Kubetzka, R. Wiesendanger, G. Bihlmayer, S. Blügel, Spontaneous atomic-scale magnetic skyrmion lattice in two dimensions, Nature Physics 7 (9) (2011) 713718. doi:10.1038/nphys2045.

URL http://www. nature.com/doifinder/10.1038/nphys2045

[44] C. Moreau-Luchaire, C. Moutas, N. Reyren, J. Sampaio, C. A. F. Vaz, N. Van Horne, K. Bouzehouane, K. Garcia, C. Deranlot, P. Warnicke, P. Wohlhüter, J.-M. George, M. Weigand, J. Raabe, V. Cros, A. Fert, Additive interfacial chiral interaction in multilayers for stabilization of small individual skyrmions at room temperature, Nature Nanotechnology 11 (January) (2016) 444. arXiv:1502.07853, doi:10.1038/nnano. 2015. 313.

URL http://dx.doi.org/10.1038/nnano.2015.313

[45] O. Boulle, J. Vogel, H. Yang, S. Pizzini, D. d. S. Chaves, A. Locatelli, T. O. M. A. Sala, L. D. Buda-Prejbeanu, O. Klein, M. Belmeguenai, Y. Roussigné, A. Stashkevich, S. M. Chérif, L. Aballe, M. Foerster, M. Chshiev, S. Auffret, I. M. Miron, G. Gaudin, Room temperature chiral magnetic skyrmion in ultrathin magnetic nanostructures, Nature nanotechnology 11 (January) (2016) 449. arXiv:1601.02278, doi:10.1038/NNANO.2015.315. 
[46] Y. Tokunaga, X. Z. Yu, J. S. White, H. M. Rønnow, D. Morikawa, Y. Taguchi, Y. Tokura, A new class of chiral materials hosting magnetic skyrmions beyond room temperature, Nature Communications 6 (May) (2015) 7638. arXiv:1503.05651, doi:10.1038/ncomms8638.

URL http://www.ncbi.nlm.nih.gov/pubmed/26134284\{\%\}5Cnhttp: //www.nature.com/doifinder/10.1038/ncomms8638\{\%\}5Cnhttp:

//www.nature.com/doifinder/10.1038/ncomms8638

[47] G. Chen, A. T. N'Diaye, S. P. Kang, H. Y. Kwon, C. Won, Y. Wu, Z. Q. Qiu, A. K. Schmid, Unlocking Bloch-type chirality in ultrathin magnets through uniaxial strain., Nature communications 6 (2015) 6598. arXiv: arXiv:1011.1669v3, doi:10.1038/ncomms7598. URL http://www.ncbi.nlm.nih.gov/pubmed/25798953

[48] S. D. Pollard, J. A. Garlow, J. Yu, Z. Wang, Y. Zhu, H. Yang, Observation of stable Néel skyrmions in $\mathrm{Co} / \mathrm{Pd}$ multilayers with Lorentz transmission electron microscopy, Nature Communications 8 (2017) 14761. arXiv: 1702.00113, doi:10.1038/ncomms14761. URL http://dx.doi.org/10.1038/ncomms14761

[49] A. Fert, P. M. Levy, Role of Anisotropic Exchange Interactions in Determining the Properties of Spin-Glasses, Phys. Rev. Lett. 44 (23) (1980) 1538 .

[50] G. Chen, J. Zhu, A. Quesada, J. Li, A. T. N. Diaye, Y. Huo, T. P. Ma, Y. Chen, H. Y. Kwon, C. Won, Z. Q. Qiu, A. K. Schmid, Y. Z. Wu, Novel Chiral Magnetic Domain Wall Structure in Fe/Ni/Cu(001) Films, Phys. Rev. Lett. 177204 (April) (2013) 1-5. doi:10.1103/PhysRevLett.110. 177204.

[51] G. Chen, T. Ma, A. T. N'Diaye, H. Kwon, C. Won, Y. Wu, A. K. Schmid, Tailoring the chirality of magnetic domain walls by interface engineering, Nature Communications 4 (2013) 2671. URL http://dx.doi.org/10.1038/ncomms367110.1038/ncomms3671

[52] J. Tetienne, T. Hingant, L. J. Martinez, S. Rohart, A. Thiaville, L. Herrera Diez, K. Garcia, J. P. Adam, J.-V. Kim, J. F. Roch, I. M. Miron, G. Gaudin, L. Vila, B. Ocker, D. Ravelosona, V. Jacques, The nature of domain walls in ultrathin ferromagnets revealed by scanning nanomagnetometry, Nature Communications 6 (2015) 6733. doi:10.1038/ ncomms 7733 .

[53] M. Bode, M. Heide, K. von Bergmann, P. Ferriani, S. Heinze, G. Bihlmayer, A. Kubetzka, O. Pietzsch, S. Blügel, R. Wiesendanger, Chiral magnetic order at surfaces driven by inversion asymmetry., Nature 447 (7141) (2007) 190-3. doi:10.1038/nature05802. URL http://www.ncbi.nlm.nih.gov/pubmed/17495922 
[54] P. Ferriani, K. Von Bergmann, E. Y. Vedmedenko, S. Heinze, M. Bode, M. Heide, G. Bihlmayer, S. Blügel, R. Wiesendanger, Atomic-scale Spin spiral with a unique rotational sense: Mn monolayer on W(001), Physical Review Letters 101 (2) (2008) 027201. doi:10.1103/PhysRevLett.101. 027201.

[55] M. Menzel, Y. Mokrousov, R. Wieser, J. E. Bickel, E. Vedmedenko, S. Blügel, S. Heinze, K. von Bergmann, A. Kubetzka, R. Wiesendanger, Information Transfer by Vector Spin Chirality in Finite Magnetic Chains, Phys. Rev. Lett. 108 (19) (2012) 197204. doi:10.1103/PhysRevLett. 108.197204. URL http://link.aps.org/doi/10.1103/PhysRevLett.108.197204

[56] Y. Yoshida, S. Schröder, P. Ferriani, D. Serrate, A. Kubetzka, K. von Bergmann, S. Heinze, R. Wiesendanger, Conical Spin-Spiral State in an Ultrathin Film Driven by Higher-Order Spin Interactions, Phys. Rev. Lett. 108 (8) (2012) 087205. doi:10.1103/PhysRevLett.108.087205. URL http://link.aps.org/doi/10.1103/PhysRevLett.108.087205

[57] R. Wiesendanger, Nanoscale magnetic skyrmions in metallic films and multilayers: a new twist for spintronics, Nature Reviews Materials 1 (2016) 16044. doi:10.1038/natrevmats.2016.44. URL http://www.nature.com/articles/natrevmats201644

[58] Y. Onose, T. Ideue, H. Katsura, Y. Shiomi, N. Nagaosa, Y. Tokura, Observation of the magnon Hall effect., Science (New York, N.Y.) 329 (5989) (2010) 297-9. doi:10.1126/science.1188260. URL http://www.ncbi.nlm.nih.gov/pubmed/20647460

[59] A. Manchon, P. B. Ndiaye, J.-h. Moon, H.-w. Lee, K.-j. Lee, Magnonmediated Dzyaloshinskii-Moriya torque in homogeneous ferromagnets, Phys. Rev. B 90 (2014) 224403. doi:10.1103/PhysRevB.90.224403.

[60] A. A. Kovalev, V. Zyuzin, Spin torque and Nernst effects in Dzyaloshinskii-Moriya ferromagnets, Physical Review B - Condensed Matter and Materials Physics 93 (16) (2016) 161106. arXiv:arXiv:1509. 05847v3, doi:10.1103/PhysRevB.93.161106.

[61] I. A. Sergienko, E. Dagotto, Role of the Dzyaloshinskii-Moriya interaction in multiferroic perovskites, Physical Review B - Condensed Matter and Materials Physics 73 (9) (2006) 094434. arXiv:0508075, doi:10.1103/ PhysRevB.73.094434.

[62] J. Z. Zhao, X. Q. Wang, T. Xiang, Z. B. Su, L. Yu, Effects of the \{Dzyaloshinskii-Moriya\} Interaction on \{Low-Energy Magnetic Excitations in Copper Benzoate, Physical Review Letters 90 (20) (2003) 207204. arXiv:0212410v2, doi:10.1103/PhysRevLett.90.207204. URL http://link.aps.org/doi/10.1103/PhysRevLett.90.207204 
[63] H. De Raedt, S. Miyashita, K. Michielsen, M. Machida, DzyaloshinskiiMoriya interactions and adiabatic magnetization dynamics in molecular magnets, Physical Review B - Condensed Matter and Materials Physics 70 (6) (2004) 064401. doi:10.1103/PhysRevB.70.064401.

[64] P. Kurz, G. Bihlmayer, K. Hirai, S. Blügel, Three-Dimensional Spin Structure on a Two-Dimensional Lattice: $\mathrm{Mn} / \mathrm{Cu}(111)$, Physical Review Letters 86 (6) (2001) 1106-1109. doi:10.1103/PhysRevLett.86.1106.

[65] L. Balents, Spin liquids in frustrated magnets., Nature 464 (7286) (2010) 199-208. arXiv:9904169, doi:10.1038/nature08917. URL http://www.ncbi.nlm.nih.gov/pubmed/20220838

[66] M. Hoffmann, J. Weischenberg, B. Dupe, F. Freimuth, P. Ferriani, Y. Mokrousov, S. Heinze, Topological orbital magnetization and emergent Hall effect of an atomic-scale spin lattice at a surface, Physical Review B 92 (2) (2015) 020401(R). arXiv:1503.01885v2, doi:10.1103/PhysRevB. 92.020401.

[67] J. Kim, J. Sinha, S. Mitani, M. Hayashi, S. Takahashi, S. Maekawa, M. Yamanouchi, H. Ohno, Anomalous temperature dependence of currentinduced torques in $\mathrm{CoFeB} / \mathrm{MgO}$ heterostructures with Ta-based underlayers, Phys. Rev. B 89 (2014) 174424. doi:10.1103/PhysRevB.89.174424.

[68] I. Garate, M. Franz, Magnetoelectric response of the time-reversal invariant helical metal, Phys. Rev. B 81 (17) (2010) 172408. doi:10.1103/ PhysRevB.81.172408. URL http://link.aps.org/doi/10.1103/PhysRevB.81.172408

[69] A. Thiaville, S. Rohart, É. Jué, V. Cros, A. Fert, Dynamics of Dzyaloshinskii domain walls in ultrathin magnetic films, Europhysics Letters 100 (5) (2012) 57002. doi:10.1209/0295-5075/100/57002.

URL http://stacks.iop.org/0295-5075/100/i=5/a=57002?key= crossref .2efecb4b4825b9f99211cd2da9f0957b

[70] V. Kashid, T. Schena, B. Zimmermann, Y. Mokrousov, S. Blügel, V. Shah, H. G. Salunke, Dzyaloshinskii-Moriya interaction and chiral magnetism in 3d5d zigzag chains: Tight-binding model and ab initio calculations, Phys. Rev. B 90 (5) (2014) 054412. doi:10.1103/PhysRevB.90.054412. URL http://link.aps.org/doi/10.1103/PhysRevB.90.054412

[71] J. C. Slater, G. F. Koster, Simplified LCAO method for the periodic potential problem, Physical Review 94 (6) (1954) 1498-1524. doi:10. 1103/PhysRev.94.1498.

[72] A. T. Costa, R. B. Muniz, S. Lounis, A. Klautau, D. L. Mills, Spin-orbit coupling and spin waves in ultrathin ferromagnets: The spin-wave Rashba effect, Phys. Rev. B 82 (1) (2010) 014428. doi:10.1103/PhysRevB. 82. 014428.

URL http://link.aps.org/doi/10.1103/PhysRevB.82.014428 
[73] H. Yang, S. Rohart, A. Fert, M. Chshiev, Anatomy of DzyaloshinskiiMoriya Interaction at Co-Pt Interfaces, Phys. Rev. Lett. 115 (2015) 267210. doi:10.1103/PhysRevLett.115.267210.

[74] C. Herring, Magnetism: Exchange interactions among itinerant electrons, in: G. T. Rado, H. Suhl (Eds.), Magnetism, Academic Press, 1966, p. 407.

[75] L. Sandratskii, Symmetry analysis of electronic states for crystals with spiral magnetic order. I. General properties, Journal of Physics: Condensed Matter 3 (1991) 8565.

URL http://iopscience.iop.org/0953-8984/3/44/004

[76] P. Kurz, F. Förster, L. Nordström, G. Bihlmayer, S. Blügel, Ab initio treatment of noncollinear magnets with the full-potential linearized augmented plane wave method, Phys. Rev. B 69 (2) (2004) 024415. doi:10.1103/PhysRevB.69.024415. URL http://link.aps.org/doi/10.1103/PhysRevB.69.024415

[77] M. Heide, G. Bihlmayer, S. Blügel, Dzyaloshinskii-Moriya interaction accounting for the orientation of magnetic domains in ultrathin films: Fe/W(110), Phys. Rev. B 78 (14) (2008) 140403. doi:10.1103/PhysRevB. 78.140403. URL http://link.aps.org/doi/10.1103/PhysRevB.78.140403

[78] M. Heide, G. Bihlmayer, S. Blügel, Describing Dzyaloshinskii-Moriya spirals from first principles, Physica B: Condensed Matter 404 (18) (2009) 2678-2683. doi:10.1016/j.physb.2009.06.070.

[79] F. Freimuth, R. Bamler, Y. Mokrousov, A. Rosch, Phase-space Berry phases in chiral magnets: Dzyaloshinskii-Moriya interaction and the charge of skyrmions, Phys. Rev. B 88 (21) (2013) 214409. doi:10.1103/ PhysRevB.88.214409. URL http://link.aps.org/doi/10.1103/PhysRevB.88.214409

[80] F. Freimuth, S. Blügel, Y. Mokrousov, Berry phase theory of Dzyaloshinskii-Moriya interaction and spin-orbit torques., Journal of physics. Condensed matter : an Institute of Physics journal 26 (10) (2014) 104202. doi:10.1088/0953-8984/26/10/104202. URL http://www.ncbi.nlm.nih.gov/pubmed/24552898

[81] T. Kikuchi, T. Koretsune, R. Arita, G. Tatara, Dzyaloshinskii-Moriya Interaction as a Consequence of a Doppler Shift due to Spin-Orbit-Induced Intrinsic Spin Current, Physical Review Letters 116 (24) (2016) 247201. arXiv:1602.06481, doi:10.1103/PhysRevLett.116.247201.

[82] M. Bode, E. Y. Vedmedenko, K. von Bergmann, A. Kubetzka, P. Ferriani, S. Heinze, R. Wiesendanger, Atomic spin structure of antiferromagnetic domain walls., Nature materials 5 (2006) 477-481. doi: $10.1038 /$ nmat 1646 . 
[83] J. Gayles, F. Freimuth, T. Schena, G. Lani, P. Mavropoulos, R. A. Duine, S. Blügel, J. Sinova, Y. Mokrousov, Dzyaloshinskii-Moriya Interaction and Hall Effects in the Skyrmion Phase of Mn1-xFexGe, Physical Review Letters 115 (3) (2015) 1-6. arXiv:1503.04842v1, doi: 10.1103/PhysRevLett.115.036602.

[84] A. Belabbes, G. Bihlmayer, S. Blügel, A. Manchon, Oxygen-enabled control of Dzyaloshinskii-Moriya Interaction in ultra-thin magnetic films, Scientific Reports 6 (April) (2016) 24634. arXiv:1511.01614, doi: 10.1038/srep24634.

URL http://arxiv.org/abs/1511.01614\{\%\}5Cnhttp://www. nature. com/articles/srep24634

[85] J. P. Perdew, A. Zunger, Self-interaction correction to density-functional approximations for many-electron systems, Physical Review B 23 (10) (1981) 5048-5079. arXiv:0706.3359, doi:10.1103/PhysRevB.23.5048.

[86] E. Wimmer, H. Krakauer, M. Weinert, A. J. Freeman, Full-potential selfconsistent linearized-augmented-plane-wave method for calculating the electronic structure of molecules and surfaces: O2 molecule, Physical Review B 24 (2) (1981) 864-875. doi:10.1103/PhysRevB.24.864.

[87] http://www.flapw.de.

[88] F. Hund, Zur Deutung der Molekelspektren. I, Zeitschrift f\{ü $\} r$ Physik 40 (10) (1927) 742-764. doi:10.1007/BF01400234. URL http://dx.doi.org/10.1007/BF01400234

[89] Y. O. Kvashnin, R. Cardias, A. Szilva, I. Di Marco, M. I. Katsnelson, A. I. Lichtenstein, L. Nordström, A. B. Klautau, O. Eriksson, Microscopic Origin of Heisenberg and Non-Heisenberg Exchange Interactions in Ferromagnetic bcc Fe, Physical Review Letters 116 (21) (2016) 1-5. arXiv:1510.01872, doi:10.1103/PhysRevLett.116.217202.

[90] S. Kim, K. Ueda, G. Go, P.-h. Jang, K.-j. Lee, Microscopic Origin of Interfacial Dzyaloshinskii-Moriya Interaction, arXiv preprint arXiv:1704.02900.

[91] K.-S. Ryu, S.-H. Yang, L. Thomas, S. S. P. Parkin, Chiral spin torque arising from proximity-induced magnetization., Nature Communications 5 (May) (2014) 3910. doi:10.1038/ncomms4910. URL http://www.ncbi.nlm.nih.gov/pubmed/24852680

[92] B. Schweflinghaus, B. Zimmermann, M. Heide, G. Bihlmayer, S. Blügel, Role of Dzyaloshinskii-Moriya interaction for magnetism in transitionmetal chains at Pt step edges, Physical Review B 94 (2) (2016) 1-19. arXiv:1603.01578, doi:10.1103/PhysRevB.94.024403.

[93] P. Ferriani, S. Heinze, G. Bihlmayer, S. Blügel, Unexpected trend of magnetic order of $3 \mathrm{~d}$ transition-metal monolayers on W(001), Physical Review B - Condensed Matter and Materials Physics 72 (2) (2005) 024452. doi:10.1103/PhysRevB.72.024452. 
[94] P. Ruiz-Diaz, T. R. Dasa, V. S. Stepanyuk, Tuning magnetic anisotropy in metallic multilayers by surface charging: An ab initio study, Physical Review Letters 110 (26) (2013) 267203. doi:10.1103/PhysRevLett.110. 267203.

[95] O. Cespedes, M. Wheeler, T. Moorsom, M. Viret, Unexpected magnetic properties of gas-stabilized platinum nanostructures in the tunneling regime, Nano Letters 15 (1) (2015) 45-50. doi:10.1021/nl504254d.

[96] G. Yu, P. Upadhyaya, Q. Shao, H. Wu, G. Yin, X. Li, C. He, W. Jiang, X. Han, P. K. Amiri, K. L. Wang, Room-temperature skyrmion shift device for memory application, Nano Letters 17 (1) (2017) 261-268. doi: 10.1021/acs. nanolett.6b04010.

[97] J. R. Yuan, X. H. Yan, Y. Xiao, Y. D. Guo, C. J. Dai, Noncollinear magnetic order induced by Dzyaloshinskii-Moriya interaction in oxygenassisted Pt nanojunction, Nanotechnology 27 (2016) 475202. doi:10. 1088/0957-4484/27/47/475202.

[98] A. Manchon, C. Ducruet, L. Lombard, S. Auffret, B. Rodmacq, B. Dieny, S. Pizzini, J. Vogel, V. Uhlir, M. Hochstrasser, G. Panaccione, Analysis of oxygen induced anisotropy crossover in $\mathrm{Pt} / \mathrm{Co} / \mathrm{MOx}$ trilayers, Journal of Applied Physics 104 (4) (2008) 043914. doi:10.1063/1.2969711. URL http://scitation.aip.org/content/aip/journal/jap/104/4/ 10.1063/1.2969711

[99] B. Rodmacq, A. Manchon, C. Ducruet, S. Auffret, B. Dieny, Influence of thermal annealing on the perpendicular magnetic anisotropy of Pt/Co/AlOx trilayers, Physical Review B 79 (2) (2009) 024423. doi: 10.1103/PhysRevB.79.024423.

URL http://link.aps.org/doi/10.1103/PhysRevB.79.024423

[100] S. Monso, B. Rodmacq, S. Auffret, G. Casali, F. Fettar, B. Gilles, B. Dieny, P. Boyer, Crossover from in-plane to perpendicular anisotropy in $\mathrm{Pt} / \mathrm{CoFe} / \mathrm{AlO}[\mathrm{sub} \mathrm{x}]$ sandwiches as a function of $\mathrm{Al}$ oxidation: A very accurate control of the oxidation of tunnel barriers, Applied Physics Letters 80 (22) (2002) 4157. doi:10.1063/1.1483122.

URL http://scitation.aip.org/content/aip/journal/apl/80/22/ $10.1063 / 1.1483122$

[101] L. E. Nistor, B. Rodmacq, C. Ducruet, C. Portemont, I. L. Prejbeanu, B. Dieny, Correlation between perpendicular anisotropy and magnetoresistance in magnetic tunnel junctions, IEEE Transactions on Magnetics 46 (6) (2010) 1412-1415. doi:10.1109/TMAG.2010.2045641.

[102] X. Qiu, K. Narayanapillai, Y. Wu, P. Deorani, D.-H. Yang, W.-s. Noh, J.-h. Park, K.-J. Lee, H.-w. Lee, H. Yang, Spinorbit-torque engineering via oxygen manipulation, Nature Nanotechnology 10 (4) (2015) 333-338. doi:10.1038/nnano.2015.18. 
URL http://dx.doi.org/10.1038/nnano.2015.18\{\%\}5Cnhttp:

//dx.doi.org/10.1038/nnano.2015.18\{\%\}5Cn10.1038/nnano. 2015.18\{\%\}5Cnhttp://www. nature.com/nnano/journal/v10/n4/ abs/nnano.2015.18.html\{\#\}supplementary-information

[103] O. Krupin, G. Bihlmayer, K. M. Döbrich, J. E. Prieto, K. Starke, S. Gorovikov, S. Blügel, S. Kevan, G. Kaindl, Rashba effect at the surfaces of rare-earth metals and their monoxides, New Journal of Physics 11 (1) (2009) 013035. doi:10.1088/1367-2630/11/1/013035. URL http://stacks.iop.org/1367-2630/11/i=1/a=013035?key= crossref.2c5c74413170ec4f42b5325a4b4a1df2

[104] Z. Yuan, P. J. Kelly, Spin-orbit-coupling induced torque in ballistic domain walls: Equivalence of charge-pumping and nonequilibrium magnetization formalisms, Physical Review B - Condensed Matter and Materials Physics 93 (22) (2016) 224415. arXiv:1602.06676, doi:10.1103/PhysRevB.93. 224415.

[105] B. Dupé, G. Bihlmayer, S. Blügel, S. Heinze, Engineering skyrmions in transition-metal multilayers for spintronics, Nature Communications 7 (2016) 11779. arXiv:1503.08098, doi:10.1038/ncomms11779. URL http://arxiv.org/abs/1503.08098

[106] H. Zhang, A. Soon, B. Delley, C. Stampfl, Stability, structure, and electronic properties of chemisorbed oxygen and thin surface oxides on $\operatorname{Ir}(111)$, Physical Review B - Condensed Matter and Materials Physics 78 (4) (2008) 045436. doi:10.1103/PhysRevB.78.045436.

[107] I. M. Miron, G. Gaudin, S. Auffret, B. Rodmacq, A. Schuhl, S. Pizzini, J. Vogel, P. Gambardella, Current-driven spin torque induced by the Rashba effect in a ferromagnetic metal layer., Nature Materials 9 (3) (2010) 230-4. doi:10.1038/nmat2613. URL http://www.ncbi.nlm.nih.gov/pubmed/20062047

[108] L. Liu, T. Moriyama, D. C. Ralph, R. A. Buhrman, Spin-Torque Ferromagnetic Resonance Induced by the Spin Hall Effect, Phys. Rev. Lett. 106 (3) (2011) 036601. doi:10.1103/PhysRevLett.106.036601. URL http://link.aps.org/doi/10.1103/PhysRevLett.106.036601

[109] J. Kim, J. Sinha, M. Hayashi, M. Yamanouchi, S. Fukami, T. Suzuki, S. Mitani, H. Ohno, Layer thickness dependence of the current-induced effective field vector in $\mathrm{Ta}-\mathrm{CoFeB}-\mathrm{MgO}$., Nature Materials 12 (3) (2013) 240-5. doi:10.1038/nmat3522.

URL http://www.ncbi.nlm.nih.gov/pubmed/23263641

[110] A. Chernyshov, M. Overby, X. Liu, J. K. Furdyna, Y. Lyanda-Geller, L. P. Rokhinson, Evidence for reversible control of magnetization in a ferromagnetic material by means of spinorbit magnetic field, Nature Physics 5 (9) 
(2009) 656-659. doi:10.1038/nphys1362.

URL http://www . nature.com/doifinder/10.1038/nphys1362

[111] D. Fang, H. Kurebayashi, J. Wunderlich, K. Výborný, L. P. Zârbo, R. P. Campion, A. Casiraghi, B. L. Gallagher, T. Jungwirth, A. J. Ferguson, Spin-orbit-driven ferromagnetic resonance., Nature Nanotechnology 6 (7) (2011) 413-7. doi:10.1038/nnano.2011.68.

URL http://www.ncbi.nlm.nih.gov/pubmed/21602814

[112] H. Kurebayashi, J. Sinova, D. Fang, A. Irvine, T. D. Skinner, J. Wunderlich, V. Novák, R. P. Campion, B. L. Gallagher, E. K. Vehstedt, L. P. Zârbo, K. Výborný, A. J. Ferguson, T. Jungwirth, An antidamping spinorbit torque originating from the Berry curvature., Nature Nanotechnology 9 (3) (2014) 211-7. doi:10.1038/nnano.2014.15.

URL http://www.ncbi.nlm.nih.gov/pubmed/24584275

[113] X. Fan, J. Wu, Y. Chen, M. J. Jerry, H. Zhang, J. Q. Xiao, Observation of the nonlocal spin-orbital effective field, Nature Communications 4 (2013) 1799. doi:10.1038/ncomms2709.

URL http://www.nature.com/doifinder/10.1038/ncomms2709

[114] C. O. Avci, K. Garello, C. Nistor, S. Godey, B. Ballesteros, A. Mugarza, A. Barla, M. Valvidares, E. Pellegrin, A. Ghosh, I. M. Miron, O. Boulle, S. Auffret, G. Gaudin, P. Gambardella, Fieldlike and antidamping spinorbit torques in as-grown and annealed $\mathrm{Ta} / \mathrm{CoFeB} / \mathrm{MgO}$ layers, Phys. Rev. B 89 (2014) 214419. arXiv:1402.0986, doi:10.1103/PhysRevB. 89.214419.

[115] X. Qiu, W. Legrand, P. He, Y. Wu, J. Yu, R. Ramaswamy, A. Manchon, H. Yang, Enhanced Spin-Orbit Torque via Modulation of Spin Current Absorption, Phys. Rev. Lett. 117 (November) (2016) 217206. doi:10. 1103/PhysRevLett.117.217206.

[116] Y. Wen, J. Wu, P. Li, Q. Zhang, Y. Zhao, A. Manchon, J. Q. Xiao, X. Zhang, Temperature dependence of spin-orbit torques in $\mathrm{Cu}-\mathrm{Au}$ alloys, Physical Review B 95 (10) (2017) 104403. doi:10.1103/PHYSREVB.95.104403.

URL http://journals.aps.org/prb/abstract/10.1103/PhysRevB . 95.104403

[117] S. Emori, U. Bauer, S. Woo, G. S. D. Beach, Large voltage-induced modification of spin-orbit torques in $\mathrm{Pt} / \mathrm{Co} / \mathrm{GdOx}$, Applied Physics Letters 105 (22) (2014) 222401. doi:10.1063/1.4903041.

[118] S. Emori, T. Nan, A. M. Belkessam, X. Wang, A. D. Matyushov, C. J. Babroski, Y. Gao, H. Lin, N. X. Sun, Interfacial spin-orbit torque without bulk spin-orbit coupling, Physical Review B 93 (18) (2016) 180402. arXiv: arXiv:1601.07687v2, doi:10.1103/PhysRevB.93.180402. URL http://link.aps.org/doi/10.1103/PhysRevB.93.180402 
[119] M. Jamali, K. Narayanapillai, X. Qiu, L. M. Loong, A. Manchon, H. Yang, Spin-orbit torques in $\mathrm{Co} / \mathrm{Pd}$ multilayer nanowires, Physical Review Letters 111 (24) (2013) 246602.

[120] D. Bang, J. Yu, X. Qiu, Y. Wang, H. Awano, A. Manchon, H. Yang, Enhancement of spin Hall effect induced torques for current-driven magnetic domain wall motion: Inner interface effect, Physical Review B 93 (17) (2016) 174424. doi:10.1103/PhysRevB.93.174424.

URL http://link.aps.org/doi/10.1103/PhysRevB.93.174424

[121] Y. Kajiwara, K. Harii, S. Takahashi, J.-i. Ohe, K. Uchida, M. Mizuguchi, H. Umezawa, H. Kawai, K. Ando, K. Takanashi, S. Maekawa, E. Saitoh, Transmission of electrical signals by spin-wave interconversion in a magnetic insulator., Nature 464 (7286) (2010) 262-6. doi:10.1038/ nature08876. URL http://www.ncbi.nlm.nih.gov/pubmed/20220845

[122] A. Hamadeh, O. A. Kelly, C. Hahn, H. Meley, R. Bernard, A. H. Molpeceres, V. V. Naletov, A. Anane, V. Cros, S. O. Demokritov, J. L. Prieto, M. Mu, G. D. Loubens, O. Klein, Electronic control of the spin-wave damping in a magnetic insulator, Phys. Rev. Lett. 113 (2014) 197203.

[123] C. O. Avci, A. Quindeau, C.-F. Pai, M. Mann, L. Caretta, A. S. Tang, M. C. Onbasli, C. A. Ross, G. S. D. Beach, Current-induced switching in a magnetic insulator, Nature Materials 16 (November) (2017) 309. doi: $10.1038 /$ nmat 4812 .

URL http://dx.doi.org/10.1038/nmat4812\{\%\}5Cnhttp://10. 1038/nmat4812\{\%\}5Cnhttp://www. nature.com/nmat/journal/vaop/ ncurrent/abs/nmat4812.html\{\#\}supplementary-information

[124] A. R. Mellnik, J. S. Lee, A. Richardella, J. L. Grab, P. J. Mintun, M. H. Fischer, A. Vaezi, A. Manchon, E.-a. Kim, N. Samarth, D. C. Ralph, Spintransfer torque generated by a topological insulator, Nature 511 (7510) (2014) 449-451. doi:10.1038/nature13534. URL http://www .nature.com/doifinder/10.1038/nature13534

[125] Y. Fan, P. Upadhyaya, X. Kou, M. Lang, S. Takei, Z. Wang, J. Tang, L. He, L.-T. Chang, M. Montazeri, G. Yu, W. Jiang, T. Nie, R. N. Schwartz, Y. Tserkovnyak, K. L. Wang, Magnetization switching through giant spin-orbit torque in a magnetically doped topological insulator heterostructure., Nature materials 13 (7) (2014) 699-704. doi:10.1038/ nmat3973.

URL http://www.ncbi.nlm.nih.gov/pubmed/24776536

[126] Y. Wang, P. Deorani, K. Banerjee, N. Koirala, M. Brahlek, S. Oh, H. Yang, Topological surface states originated spin-orbit torques in Bi2Se3, Physical Review Letters 114 (25) (2015) 257202. arXiv:1505.07937, doi: 10.1103/PhysRevLett.114.257202. 
[127] Y. Fan, X. Kou, P. Upadhyaya, Q. Shao, L. Pan, M. Lang, X. Che, J. Tang, M. Montazeri, K. Murata, L.-T. Chang, M. Akyol, G. Yu, T. Nie, K. L. Wong, J. Liu, Y. Wang, Y. Tserkovnyak, K. L. Wang, Electric-field control of spin-orbit torque in a magnetically doped topological insulator, Nature Nanotechnology 11 (4) (2016) 352. arXiv:1511.07442, doi:10.1038/ nnano.2015.294.

URL http://arxiv.org/abs/1511.07442

[128] D. MacNeill, G. M. Stiehl, M. H. D. Guimaraes, R. A. Buhrman, J. Park, D. C. Ralph, Control of spin-orbit torques through crystal symmetry in WTe2/ferromagnet bilayers, Nature Physics 1 (November). arXiv:1605. 02712, doi:10.1038/nphys3933.

URL http://arxiv.org/abs/1605.02712

[129] A. Manchon, S. Zhang, Theory of nonequilibrium intrinsic spin torque in a single nanomagnet, Phys. Rev. B 78 (2008) 212405.

[130] A. Manchon, S. Zhang, Theory of spin torque due to spin-orbit coupling, Physical Review B 79 (9) (2009) 094422. doi:10.1103/PhysRevB.79. 094422. URL http://prb.aps.org/abstract/PRB/v79/i9/e094422

[131] I. Garate, A. H. MacDonald, Influence of a transport current on magnetic anisotropy in gyrotropic ferromagnets, Phys. Rev. B 80 (13) (2009) 134403. doi:10.1103/PhysRevB.80.134403. URL http://link.aps.org/doi/10.1103/PhysRevB.80.134403

[132] P. M. Haney, H.-W. Lee, K.-J. Lee, A. Manchon, M. D. Stiles, Current induced torques and interfacial spin-orbit coupling: Semiclassical modeling, Phys. Rev. B 87 (17) (2013) 174411. doi:10.1103/PhysRevB.87.174411. URL http://link.aps.org/doi/10.1103/PhysRevB.87.174411

[133] E. van der Bijl, R. a. Duine, Current-induced torques in textured Rashba ferromagnets, Phys. Rev. B 86 (9) (2012) 094406. doi:10.1103/ PhysRevB. 86.094406. URL http://link.aps.org/doi/10.1103/PhysRevB.86.094406

[134] K.-S. Lee, D. Go, A. Manchon, P. M. Haney, M. D. Stiles, H.-W. Lee, K.-J. Lee, Angular dependence of spin-orbit spin-transfer torques, Phys. Rev. B 91 (14) (2015) 144401. doi:10.1103/PhysRevB.91.144401. URL http://link.aps.org/doi/10.1103/PhysRevB.91.144401

[135] H. Li, H. Gao, L. P. Zârbo, K. Výborný, X. Wang, I. Garate, F. Dogan, A. Cejchan, J. Sinova, T. Jungwirth, A. Manchon, Intraband and interband spin-orbit torques in noncentrosymmetric ferromagnets, Phys. Rev. B 91 (13) (2015) 134402. doi:10.1103/PhysRevB.91.134402. URL http://link.aps.org/doi/10.1103/PhysRevB.91.134402 
[136] H. B. M. Saidaoui, A. Manchon, Spin-Swapping Transport and Torques in Ultrathin Magnetic Bilayers, Physical Review Letters 117 (3) (2016) 036601. arXiv:1511.03454, doi:10.1103/PhysRevLett.117.036601. URL http://arxiv.org/abs/1511.03454

[137] M. I. D'yakonov, V. I. Perel', Possibility of orienting electron spins with current (1971). doi:10.1063/1.1614421.

URL http://www.jetpletters.ac.ru/ps/1587/article\{_\}24366. shtml

[138] M. Dyakonov, V. I. Perel, Current-induced spin orientation of electrons in semiconductors, Physics Letters 35 (6) (1971) 459-460. doi:10.1016/0375-9601(71)90196-4.

URL http://linkinghub.elsevier.com/retrieve/pii/ 0375960171901964

[139] J. Hirsch, Spin Hall Effect, Phys. Rev. Lett. 83 (9) (1999) 1834-1837. doi:10.1103/PhysRevLett.83.1834. URL http://link.aps.org/doi/10.1103/PhysRevLett.83.1834

[140] S. Zhang, Spin hall effect in the presence of spin diffusion, Phys. Rev. Lett. 85 (2) (2000) 393-6.

URL http://www.ncbi.nlm.nih.gov/pubmed/10991291

[141] Y. K. Kato, R. C. Myers, A. C. Gossard, D. D. Awschalom, Observation of the spin Hall effect in semiconductors., Science (New York, N.Y.) 306 (2004) 1910-1913. doi:10.1126/science.1105514. URL http://www.ncbi.nlm.nih.gov/pubmed/15539563

[142] J. Wunderlich, B. Kaestner, J. Sinova, T. Jungwirth, Experimental observation of the spin-Hall effect in a two dimensional spin-orbit coupled semiconductor system, Phys. Rev. Lett. 94 (2005) 047204. doi: 10.1103/PhysRevLett.94.047204. URL http://link.aps.org/doi/10.1103/PhysRevLett.94.047204

[143] S. O. Valenzuela, M. Tinkham, Direct electronic measurement of the spin Hall effect., Nature 442 (7099) (2006) 176-9. doi:10.1038/nature04937. URL http://www.ncbi.nlm.nih.gov/pubmed/16838016

[144] T. Kimura, Y. Otani, T. Sato, S. Takahashi, S. Maekawa, RoomTemperature Reversible Spin Hall Effect, Phys. Rev. Lett. 98 (15) (2007) 156601. doi:10.1103/PhysRevLett.98.156601. URL http://link.aps.org/doi/10.1103/PhysRevLett.98.156601

[145] S. Takahashi, S. Maekawa, Spin Current in Metals and Superconductors, Journal of the Physical Society of Japan 77 (3) (2008) 031009. doi: 10.1143/JPS J.77.031009.

URL http://journals.jps.jp/doi/abs/10.1143/JPSJ.77.031009 
[146] G. Vignale, Ten Years of Spin Hall Effect, Journal of Superconductivity and Novel Magnetism 23 (1) (2010) 3-10. doi:10.1007/ s10948-009-0547-9. URL http://link.springer.com/10.1007/s10948-009-0547-9

[147] A. Hoffmann, Spin Hall Effects in Metals, IEEE Transactions on Magnetics 49 (10) (2013) 5172-5193. doi:10.1109/TMAG.2013.2262947. URL http://ieeexplore.ieee.org/lpdocs/epic03/wrapper.htm? arnumber $=6516040$

[148] T. Jungwirth, J. Wunderlich, K. Olejník, Spin Hall effect devices., Nature Materials 11 (5) (2012) 382-90. doi:10.1038/nmat3279. URL http://www.ncbi.nlm.nih.gov/pubmed/22522638

[149] J. Sinova, S. O. Valenzuela, J. Wunderlich, C. H. Back, T. Jungwirth, Spin Hall effect, Rev. Mod. Phys. 87 (2015) 1213. arXiv:1411.3249v1.

[150] J. Wunderlich, B.-G. Park, A. Irvine, L. P. Zârbo, E. Rozkotová, P. Nemec, V. Novák, J. Sinova, T. Jungwirth, Spin Hall effect transistor., Science (New York, N.Y.) 330 (6012) (2010) 1801-4. doi:10.1126/science. 1195816. URL http://www.ncbi.nlm.nih.gov/pubmed/21205664

[151] T. Tanaka, H. Kontani, M. Naito, T. Naito, D. Hirashima, K. Yamada, J. Inoue, Intrinsic spin Hall effect and orbital Hall effect in 4d and 5d transition metals, Phys. Rev. B 77 (16) (2008) 165117. doi:10.1103/ PhysRevB.77.165117. URL http://link.aps.org/doi/10.1103/PhysRevB.77.165117

[152] H. Kontani, T. Tanaka, D. Hirashima, K. Yamada, J. Inoue, Giant Intrinsic Spin and Orbital Hall Effects in Sr2MO4 (M=Ru, Rh, Mo), Phys. Rev. Lett. 100 (9) (2008) 096601. doi:10.1103/PhysRevLett.100.096601. URL http://link.aps.org/doi/10.1103/PhysRevLett.100.096601

[153] H. Kontani, T. Tanaka, D. Hirashima, K. Yamada, J. Inoue, Giant Orbital Hall Effect in Transition Metals: Origin of Large Spin and Anomalous Hall Effects, Phys. Rev. Lett. 102 (1) (2009) 016601. doi: 10.1103/PhysRevLett.102.016601. URL http://link.aps.org/doi/10.1103/PhysRevLett.102.016601

[154] Y. Yao, L. Kleinman, A. H. MacDonald, J. Sinova, T. Jungwirth, D.-s. Wang, E. Wang, Q. Niu, First Principles Calculation of Anomalous Hall Conductivity in Ferromagnetic bcc Fe, Phys. Rev. Lett. 92 (3) (2004) 1-4. doi:10.1103/PhysRevLett.92.037204. URL http://link.aps.org/doi/10.1103/PhysRevLett.92.037204

[155] Y. Yao, Z. Fang, Sign changes of intrinsic spin hall effect in semiconductors and simple metals: First-principles calculations, Physical Review Letters 95 (2005) 156601. arXiv:0502351, doi:10.1103/PhysRevLett. 95.156601 . 
[156] G. Y. Guo, Y. Yao, Q. Niu, Ab initio calculation of the intrinsic spin hall effect in semiconductors, Physical Review Letters 94 (2005) 226601. doi:10.1103/PhysRevLett.94.226601.

[157] G. Guo, S. Murakami, T.-W. Chen, N. Nagaosa, Intrinsic Spin Hall Effect in Platinum: First-Principles Calculations, Phys. Rev. Lett. 100 (9) (2008) 096401. doi:10.1103/PhysRevLett.100.096401. URL http://link.aps.org/doi/10.1103/PhysRevLett.100.096401

[158] M. Gradhand, D. V. Fedorov, P. Zahn, I. Mertig, Spin Hall angle versus spin diffusion length: Tailored by impurities, Phys. Rev. B 81 (24) (2010) 245109. doi:10.1103/PhysRevB.81.245109. URL http://link.aps.org/doi/10.1103/PhysRevB.81.245109

[159] M. Gradhand, D. V. Fedorov, P. Zahn, I. Mertig, Extrinsic Spin Hall Effect from First Principles, Phys. Rev. Lett. 104 (18) (2010) 186403. doi:10.1103/PhysRevLett.104.186403. URL http://link.aps.org/doi/10.1103/PhysRevLett.104.186403

[160] S. Lowitzer, D. Ködderitzsch, H. Ebert, Coherent description of the intrinsic and extrinsic anomalous Hall effect in disordered alloys on an ab initio level, Phys. Rev. Lett. 105 (26) (2010) 266604.

URL http://arxiv.org/abs/1008.2640

[161] S. Lowitzer, M. Gradhand, D. Ködderitzsch, D. V. Fedorov, I. Mertig, H. Ebert, Extrinsic and Intrinsic Contributions to the Spin Hall Effect of Alloys, Phys. Rev. Lett. 106 (5) (2011) 056601. doi:10.1103/ PhysRevLett.106.056601. URL http://link.aps.org/doi/10.1103/PhysRevLett.106.056601

[162] M. Gradhand, D. V. Fedorov, F. Pientka, P. Zahn, I. Mertig, B. L. Györffy, First-principle calculations of the Berry curvature of Bloch states for charge and spin transport of electrons, Journal of Physics: Condensed Matter 24 (2012) 213202. doi:10.1088/0953-8984/24/21/213202.

[163] M. Gradhand, D. V. Fedorov, P. Zahn, I. Mertig, Fully relativistic ab initio treatment of spin-flip scattering caused by impurities, Physical Review B - Condensed Matter and Materials Physics 81 (2010) 020403. doi: 10.1103/PhysRevB.81.020403.

[164] J. Smit, The spontaneous Hall effect in ferromagnetics II, Physica 24 (1958) 39-51.

[165] L. Berger, Side-jump mechanism for the Hall effect of Ferromagnets, Phys. Rev. B 2 (11) (1970) 4559.

[166] A. Crépieux, P. Bruno, Theory of the anomalous Hall effect from the Kubo formula and the Dirac equation, Phys. Rev. B 64 (1) (2001) 014416. doi:10.1103/PhysRevB.64.014416. URL http://link.aps.org/doi/10.1103/PhysRevB.64.014416 
[167] H. B. M. Saidaoui, Y. Otani, A. Manchon, Crossover between spin swapping and Hall effect in disordered systems, Phys. Rev. B 92 (2015) 024417. doi:10.1103/PhysRevB.92.024417.

[168] A. Fert, P. M. Levy, Spin hall effect induced by resonant scattering on impurities in metals, Physical Review Letters 106 (15) (2011) 157208. arXiv:1012.3657, doi:10.1103/PhysRevLett.106.157208.

[169] P. M. Levy, H. Yang, M. Chshiev, A. Fert, Spin Hall effect induced by Bi impurities in Cu: Skew scattering and side-jump, Phys. Rev. B 88 (21) (2013) 214432. doi:10.1103/PhysRevB.88.214432. URL http://link.aps.org/doi/10.1103/PhysRevB.88.214432

[170] B. Gu, I. Sugai, T. Ziman, G. Y. Guo, N. Nagaosa, T. Seki, K. Takanashi, S. Maekawa, Surface-Assisted Spin Hall Effect in Au Films with Pt Impurities, Phys. Rev. Lett. 105 (21) (2010) 216401. doi:10.1103/ PhysRevLett.105.216401.

URL http://link.aps.org/doi/10.1103/PhysRevLett.105.216401

[171] A. Fert, A. Friederich, A. Hamzic, Hall effect in dilute magnetic alloys, Journal of Magnetism and Magnetic Materials 24 (1981) 231-257.

[172] G. Y. Guo, S. Maekawa, N. Nagaosa, Enhanced spin hall effect by resonant skew scattering in the orbital-dependent kondo effect, Phys. Rev. Lett. 102 (3) (2009) 036401. doi:10.1103/PhysRevLett.102.036401. URL http://link.aps.org/doi/10.1103/PhysRevLett.102.036401

[173] T. Seki, Y. Hasegawa, S. Mitani, S. Takahashi, H. Imamura, S. Maekawa, J. Nitta, K. Takanashi, Giant spin Hall effect in perpendicularly spinpolarized FePt/Au devices., Nature Materials 7 (2) (2008) 125-9. doi: $10.1038 /$ nmat2098.

URL http://www.ncbi.nlm.nih.gov/pubmed/18193052

[174] T. Tanaka, H. Kontani, Giant extrinsic spin Hall effect due to rareearth impurities, New Journal of Physics 11 (1) (2009) 013023. doi: 10.1088/1367-2630/11/1/013023.

URL http://stacks.iop.org/1367-2630/11/i=1/a=013023?key= crossref.313d76dd2c5fa5ad2730c85d07a8d959

[175] P. Laczkowski, J.-C. Rojas-Sánchez, W. Savero-Torres, H. Jaffrès, N. Reyren, C. Deranlot, L. Notin, C. Beigné, A. Marty, J.-P. Attane, L. Vila, J.-M. George, A. Fert, Experimental evidences of a large extrinsic spin Hall effect in AuW alloy, Applied Physics Letters 104 (14) (2014) 142403. doi:10.1063/1.4870835.

URL http://scitation.aip.org/content/aip/journal/apl/104/14/ $10.1063 / 1.4870835$

[176] Y. Niimi, M. Morota, D. H. Wei, C. Deranlot, M. Basletic, A. Hamzic, A. Fert, Y. Otani, Extrinsic Spin Hall Effect Induced by Iridium Impurities in Copper, Phys. Rev. Lett. 106 (12) (2011) 126601. doi: 
10.1103/PhysRevLett.106.126601.

URL http://link.aps.org/doi/10.1103/PhysRevLett.106.126601

[177] Y. Niimi, Y. Kawanishi, D. H. Wei, C. Deranlot, H. X. Yang, M. Chshiev, T. Valet, A. Fert, Y. Otani, Giant Spin Hall Effect Induced by Skew Scattering from Bismuth Impurities inside Thin Film CuBi Alloys, Phys. Rev. Lett. 109 (15) (2012) 156602. doi:10.1103/PhysRevLett.109.156602. URL http://link.aps.org/doi/10.1103/PhysRevLett.109.156602

[178] S. Murakami, N. Nagaosa, S.-C. Zhang, Dissipationless Quantum Spin Current at Room Temperature, Science (New York, N.Y.) 301 (2003) 1348-1351.

[179] J. Sinova, D. Culcer, Q. Niu, N. A. Sinitsyn, T. Jungwirth, A. H. MacDonald, Universal intrinsic spin Hall effect, Physical Review Letters 92 (12) (2004) 126603. arXiv:0307663, doi:10.1103/PhysRevLett.92.126603. URL http://link.aps.org/doi/10.1103/PhysRevLett.92.126603

[180] F. Freimuth, S. Blügel, Y. Mokrousov, Anisotropic Spin Hall Effect from First Principles, Phys. Rev. Lett. 105 (24) (2010) 246602. doi:10.1103/ PhysRevLett.105.246602.

URL http://link.aps.org/doi/10.1103/PhysRevLett.105.246602

[181] C. Du, H. Wang, F. Yang, P. C. Hammel, Surprisingly large inverse spin Hall effect and systematic variation of spin- orbit coupling with d -orbital filling in 3 d transition metals, arXiv preprint arXiv:1410.1590 (2014) 117arXiv:arXiv:1410.1590.

[182] W. Zhang, M. B. Jungfleisch, W. Jiang, J. E. Pearson, A. Hoffmann, F. Freimuth, Y. Mokrousov, Spin Hall Effects in Metallic Antiferromagnets, Phys. Rev. Lett. 113 (19) (2014) 196602. doi:10.1103/ PhysRevLett.113.196602.

URL http://link.aps.org/doi/10.1103/PhysRevLett.113.196602

[183] L. Wang, R. J. H. Wesselink, Y. Liu, Z. Yuan, K. Xia, P. J. Kelly, Giant Room Temperature Interface Spin Hall and Inverse Spin Hall Effects, Physical Review Letters 116 (19) (2016) 196602. arXiv:1512.07418, doi:10.1103/PhysRevLett.116.196602.

[184] M. Lifshits, M. Dyakonov, Swapping Spin Currents: Interchanging Spin and Flow Directions, Phys. Rev. Lett. 103 (18) (2009) 186601. doi:10. 1103/PhysRevLett.103.186601.

URL http://link.aps.org/doi/10.1103/PhysRevLett.103.186601

[185] R. Shchelushkin, A. Brataas, Spin Hall effects in diffusive normal metals, Phys. Rev. B 71 (4) (2005) 045123. doi:10.1103/PhysRevB.71.045123. URL http://link.aps.org/doi/10.1103/PhysRevB.71.045123 
[186] S. Sadjina, A. Brataas, a. G. Mal'shukov, Intrinsic spin swapping, Phys. Rev. B 85 (11) (2012) 115306. doi:10.1103/PhysRevB.85.115306.

URL http://link.aps.org/doi/10.1103/PhysRevB.85.115306

[187] K. Shen, R. Raimondi, G. Vignale, Spin current swapping in the two dimensional electron gas, arXiv:1502.01128v1 (2015) 1-6arXiv:arXiv: $1502.01128 \mathrm{v} 1$.

[188] Camilla Espedal, Peter Lange, Severin Sadjina, A. G. Mal'shukov, Arne Brataas, Spin Hall effect and spin swapping in diffusive superconductors, Physical Review B 95 (5) (2017) 054509. doi:10.1103/PhysRevB.95. 054509.

[189] R. Shchelushkin, A. Brataas, Erratum: Spin Hall effects in diffusive normal metals [Phys. Rev. B 71, 045123 (2005)], Physical Review B 73 (16) (2006) 169907. doi:10.1103/PhysRevB.73.169907. URL http://link.aps.org/doi/10.1103/PhysRevB.73.169907

[190] H. Nakayama, M. Althammer, Y.-T. Chen, K. Uchida, Y. Kajiwara, D. Kikuchi, T. Ohtani, S. Geprägs, M. Opel, S. Takahashi, R. Gross, G. E. W. Bauer, S. T. B. Goennenwein, E. Saitoh, Spin Hall Magnetoresistance Induced by a Nonequilibrium Proximity Effect, Phys. Rev. Lett. 110 (20) (2013) 206601. doi:10.1103/PhysRevLett.110.206601. URL http://link.aps.org/doi/10.1103/PhysRevLett.110.206601

[191] A. Brataas, G. E. W. Bauer, P. Kelly, Non-collinear magnetoelectronics, Physics Reports 427 (4) (2006) 157-255. doi: 10.1016/j. physrep. 2006.01.001.

URL http://linkinghub.elsevier.com/retrieve/pii/ S0370157306000238

[192] M. Zwierzycki, Y. Tserkovnyak, P. J. Kelly, A. Brataas, G. E. W. Bauer, First-principles study of magnetization relaxation enhancement and spin transfer in thin magnetic films, Phys. Rev. B 71 (6) (2005) 064420. doi: 10.1103/PhysRevB.71.064420. URL http://link.aps.org/doi/10.1103/PhysRevB.71.064420

[193] Y.-T. Chen, S. Takahashi, H. Nakayama, M. Althammer, S. T. B. Goennenwein, E. Saitoh, G. E. W. Bauer, Theory of spin Hall magnetoresistance, Phys. Rev. B 87 (14) (2013) 144411. doi:10.1103/PhysRevB. 87. 144411.

URL http://link.aps.org/doi/10.1103/PhysRevB.87.144411

[194] J. Kim, P. Sheng, S. Takahashi, S. Mitani, M. Hayashi, Spin Hall Magnetoresistance in Metallic Bilayers, Physical Review Letters 116 (9) (2016) 097201. arXiv:1503.08903, doi:10.1103/PhysRevLett.116.097201.

[195] M. Weiler, M. Althammer, M. Schreier, J. Lotze, M. Pernpeintner, S. Meyer, H. Huebl, R. Gross, A. Kamra, J. Xiao, Y.-T. Chen, H. Jiao, 
G. E. W. Bauer, S. T. B. Goennenwein, Experimental Test of the Spin Mixing Interface Conductivity Concept, Phys. Rev. Lett. 111 (17) (2013) 176601. doi:10.1103/PhysRevLett.111.176601. URL http://link.aps.org/doi/10.1103/PhysRevLett.111.176601

[196] N. Vlietstra, J. Shan, V. Castel, B. J. van Wees, J. Ben Youssef, Spin-Hall magnetoresistance in platinum on yttrium iron garnet: Dependence on platinum thickness and in-plane/out-of-plane magnetization, Phys. Rev. B 87 (18) (2013) 184421. doi:10.1103/PhysRevB.87.184421. URL http://link.aps.org/doi/10.1103/PhysRevB.87.184421

[197] M. Althammer, S. Meyer, H. Nakayama, M. Schreier, S. Altmannshofer, M. Weiler, H. Huebl, S. Geprägs, M. Opel, R. Gross, D. Meier, C. Klewe, T. Kuschel, J. M. Schmalhorst, G. Reiss, L. Shen, A. Gupta, Y. T. Chen, G. E. W. Bauer, E. Saitoh, S. T. B. Goennenwein, Quantitative study of the spin Hall magnetoresistance in ferromagnetic insulator/normal metal hybrids, Phys. Rev. B 87 (2013) 224401. doi:10.1103/PhysRevB.87. 224401.

[198] S. S.-L. Zhang, G. Vignale, Nonlocal Anomalous Hall Effect, Physical Review Letters 116 (13) (2016) 136601. arXiv:1512.04146, doi:10.1103/PhysRevLett.116.136601.

URL http://arxiv.org/abs/1512.04146\{\%\}5Cnhttp://link.aps . org/doi/10.1103/PhysRevLett.116.136601

[199] a. Kobs, S. Heße, W. Kreuzpaintner, G. Winkler, D. Lott, P. Weinberger, A. Schreyer, H. P. Oepen, Anisotropic Interface Magnetoresistance in Pt/Co/Pt Sandwiches, Phys. Rev. Lett. 106 (21) (2011) 217207. doi:10.1103/PhysRevLett.106.217207. URL http://link.aps.org/doi/10.1103/PhysRevLett.106.217207

[200] Y. M. Lu, J. W. Cai, S. Y. Huang, D. Qu, B. F. Miao, C. L. Chien, Hybrid magnetoresistance in the proximity of a ferromagnet, Phys. Rev. B 87 (22) (2013) 220409. doi:10.1103/PhysRevB.87.220409.

URL http://link.aps.org/doi/10.1103/PhysRevB.87.220409

[201] C. O. Avci, K. Garello, A. Ghosh, M. Gabureac, S. F. Alvarado, P. Gambardella, Unidirectional spin Hall magnetoresistance in ferromagnet/normal metal bilayers, Nature Physics 11 (7) (2015) 570-575. arXiv:1502.0689, doi:10.1038/nphys3356.

URL http://dx.doi.org/10.1038/nphys3356\{\%\}5Cn10.1038/ nphys3356\{\%\}5Cnhttp://www . nature.com/nphys/journal/v11/n7/ abs/nphys3356.html\{\#\}supplementary-information\{\%\}5Cnhttp: //www.nature.com/doifinder/10.1038/nphys3356

[202] X. Wang, C. O. Pauyac, A. Manchon, Spin-orbit-coupled transport and spin torque in a ferromagnetic heterostructure, Phys. Rev. B 89 (5) (2014) 054405.

URL http://link.aps.org/doi/10.1103/PhysRevB.89.054405 
[203] S. S. L. Zhang, G. Vignale, S. Zhang, Anisotropic magnetoresistance driven by surface spin-orbit scattering, Physical Review B - Condensed Matter and Materials Physics 92 (2) (2015) 024412. arXiv:1504.03310, doi:10.1103/PhysRevB.92.024412.

[204] a. Kobs, S. Heße, H. Oepen, P. Weinberger, Anisotropic interface magnetoresistances in $\mathrm{Pt}(111) / \mathrm{Co} \mathrm{n} / \mathrm{Pt}(111)$, Philosophical Magazine 92 (22) (2012) 2835-2847. doi:10.1080/14786435.2012.676213. URL http://www.tandfonline.com/doi/abs/10.1080/14786435. 2012.676213

[205] C. W. Groth, M. Wimmer, A. R. Akhmerov, X. Waintal, Kwant: A software package for quantum transport, New Journal of Physics 16 (2014) 063065. arXiv:1309.2926, doi:10 .1088/1367-2630/16/6/063065.

[206] K.-W. Kim, J.-H. Moon, K.-J. Lee, H.-W. Lee, Prediction of Giant Spin Motive Force due to Rashba Spin-Orbit Coupling, Phys. Rev. Lett. 108 (21) (2012) 217202. doi:10.1103/PhysRevLett.108.217202.

URL http://link.aps.org/doi/10.1103/PhysRevLett.108.217202

[207] C.-F. Pai, M.-H. Nguyen, C. Belvin, L. H. Vilela-Leão, D. C. Ralph, R. A. Buhrman, Enhancement of perpendicular magnetic anisotropy and transmission of spin-Hall-effect-induced spin currents by a Hf spacer layer in $\mathrm{W} / \mathrm{Hf} / \mathrm{CoFeB} / \mathrm{MgO}$ layer structures, Applied Physics Letters 104 (8) (2014) 082407. doi:10.1063/1.4866965.

URL http://scitation.aip.org/content/aip/journal/apl/104/8/ $10.1063 / 1.4866965$

[208] M. H. Nguyen, D. C. Ralph, R. A. Buhrman, Spin Torque Study of the Spin Hall Conductivity and Spin Diffusion Length in Platinum Thin Films with Varying Resistivity, Physical Review Letters 116 (12) (2016) 126601. arXiv:1512.06931, doi:10.1103/PhysRevLett.116.126601.

[209] A. Matos-Abiague, R. L. Rodriguez-Suárez, Spin-orbit coupling mediated spin torque in a single ferromagnetic layer, Phys. Rev. B 80 (9) (2009) 94424. doi:10.1103/PhysRevB.80.094424.

URL http://link.aps.org/doi/10.1103/PhysRevB.80.094424

[210] X. Wang, C. O. Pauyac, A. Manchon, Rashba spin torque in ferromagnetic metal films, arXiv:1206.6726v1 arXiv 1206. arXiv:arXiv:1206.6726v1.

[211] D. A. Pesin, A. H. MacDonald, Quantum kinetic theory of current-induced torques in Rashba ferromagnets, Phys. Rev. B 86 (1) (2012) 014416. doi: 10.1103/PhysRevB.86.014416. URL http://link.aps.org/doi/10.1103/PhysRevB.86.014416

[212] A. Qaiumzadeh, R. A. Duine, M. Titov, Spin-orbit torques in twodimensional Rashba ferromagnets, Physical Review B 92 (1) (2015) 014402. arXiv:1503.06872, doi:10.1103/PhysRevB.92.014402. URL http://link.aps.org/doi/10.1103/PhysRevB.92.014402 
[213] I. Garate, M. Franz, Inverse Spin-Galvanic Effect in the Interface between a Topological Insulator and a Ferromagnet, Phys. Rev. Lett. 104 (14) (2010) 146802. doi:10.1103/PhysRevLett.104.146802.

URL http://link.aps.org/doi/10.1103/PhysRevLett.104.146802

[214] A. Sakai, H. Kohno, Spin torques and charge transport on the surface of topological insulator, Phys. Rev. B 89 (16) (2014) 165307. doi:10.1103/ PhysRevB.89.165307.

URL http://link.aps.org/doi/10.1103/PhysRevB.89.165307

[215] J. Fujimoto, H. Kohno, Transport properties of Dirac ferromagnet, Physical Review B - Condensed Matter and Materials Physics 90 (21) (2014) 214418. arXiv:1404.4741, doi:10.1103/PhysRevB.90.214418.

[216] M. H. Fischer, A. Vaezi, A. Manchon, E.-a. Kim, Spin-torque generation in topological insulator based heterostructures, Phys. Rev. B 93 (2016) 125303. doi:10.1103/PhysRevB.93.125303.

[217] P. B. Ndiaye, C. A. Akosa, M. H. Fischer, A. Vaezi, E. A. Kim, A. Manchon, Dirac spin-orbit torques and charge pumping at the surface of topological insulators, Physical Review B 96 (1) (2017) 014408. arXiv: 1509.06929, doi:10.1103/PhysRevB.96.014408.

[218] F. Freimuth, S. Blügel, Y. Mokrousov, Spin-orbit torques in Co/Pt(111) and $\mathrm{Mn} / \mathrm{W}(001)$ magnetic bilayers from first principles, Phys. Rev. B 90 (2014) 174423. doi:10.1103/PhysRevB.90.174423. URL http://link.aps.org/doi/10.1103/PhysRevB.90.174423

[219] J.-i. Inoue, G. E. W. Bauer, L. Molenkamp, Suppression of the persistent spin Hall current by defect scattering, Phys. Rev. B 70 (4) (2004) 041303. doi:10.1103/PhysRevB.70.041303. URL http://link.aps.org/doi/10.1103/PhysRevB.70.041303

[220] I. A. Ado, I. A. Dmitriev, P. M. Ostrovsky, M. Titov, Anomalous Hall effect with massive Dirac fermions, EPL (Europhysics Letters) 111 (2015) 37004. arXiv:1504.03658, doi:10.1209/0295-5075/111/37004. URL http://arxiv.org/abs/1504.03658

[221] I. A. Ado, I. A. Dmitriev, P. M. Ostrovsky, M. Titov, Anomalous Hall Effect in a 2D Rashba Ferromagnet, Physical Review Letters 117 (4) (2016) 046601. arXiv:1511.07413, doi:10.1103/PhysRevLett.117.046601.

[222] P. H. Chang, T. Markussen, S. Smidstrup, K. Stokbro, B. K. Nikolić, Nonequilibrium spin texture within a thin layer below the surface of current-carrying topological insulator Bi2Se3: A first-principles quantum transport study, Physical Review B 92 (20) (2015) 201406. arXiv: 1503.08046, doi:10.1103/PhysRevB.92.201406. 
[223] K. Garello, I. M. Miron, C. O. Avci, F. Freimuth, Y. Mokrousov, S. Blügel, S. Auffret, O. Boulle, G. Gaudin, P. Gambardella, Symmetry and magnitude of spin-orbit torques in ferromagnetic heterostructures., Nature Nanotechnology 8 (8) (2013) 587-93. doi:10.1038/nnano.2013.145. URL http://www.ncbi.nlm.nih.gov/pubmed/23892985

[224] G. Géranton, F. Freimuth, S. Blügel, Y. Mokrousov, Spin-orbit torques in $\mathrm{L}\{1\}_{-}\{0\}-\backslash$ mathrm $\{\mathrm{FePt}\} / \backslash$ mathrm $\{\mathrm{Pt}\}$ thin films driven by electrical and thermal currents, Phys. Rev. B 91 (1) (2015) 14417. doi:10.1103/ PhysRevB.91.014417. URL http://link.aps.org/doi/10.1103/PhysRevB.91.014417

[225] N. Bloembergen, R. W. Damon, Relaxation Effects in Ferromagnetic Resonance, Phys. Rev. 85 (1952) 699.

[226] P. W. Anderson, H. Suhl, Instability in the Motion of Ferromagnets at High Microwave Power Levels, Phys. Rev. 100 (1955) 1788.

[227] H. Suhl, The Nonlinear Behavior of Ferrites at High Microwave Signal Levels, Proc. IRE 44 (1956) 1270.

[228] M. Sparks, R. Loudon, C. Kittel, Ferromagnetic Relaxation. I. Theory of the Relaxation of the Uniform Precession and the Degenerate Spectrum in Insulators at Low Temperatures, Phys. Rev. 122 (3) (1961) 791-803. doi:10.1103/PhysRev.122.791. URL http://link.aps.org/doi/10.1103/PhysRev.122.791

[229] R. Arias, D. L. Mills, Extrinsic contributions to the ferromagnetic resonance response of ultrathin films, Phys. Rev. B 60 (10) (1999) 7395-7409. doi:10.1103/PhysRevB.60.7395. URL http://link.aps.org/doi/10.1103/PhysRevB.60.7395

[230] S. M. Rezende, A. Azevedo, M. A. Lucena, F. M. de Aguiar, Anomalous spin-wave damping in exchange-biased films, Phys. Rev. B 63 (21) (2001) 214418. doi:10.1103/PhysRevB.63.214418. URL http://link.aps.org/doi/10.1103/PhysRevB.63.214418

[231] H. Kurebayashi, O. Dzyapko, V. E. Demidov, D. Fang, a. J. Ferguson, S. O. Demokritov, Controlled enhancement of spin-current emission by three-magnon splitting., Nature Materials 10 (9) (2011) 660-4. doi:10. 1038/nmat3053. URL http://www.ncbi.nlm.nih.gov/pubmed/21725322

[232] A. Serga, A. V. Chumak, B. Hillebrands, YIG magnonics, Journal of Physics D: Applied Physics 43 (26) (2010) 264002. doi: $10.1088 / 0022-3727 / 43 / 26 / 264002$. URL http://stacks.iop.org/0022-3727/43/i=26/a=264002?key= crossref.6a073bfc1c8ba106fa1ba87bbfe37df3 
[233] B. Heinrich, R. Urban, G. Woltersdorf, Magnetic relaxations in metallic multilayers, IEEE Transactions on Magnetics 38 (5) (2002) 2496-2501. doi:10.1109/TMAG.2002.801906.

URL http://ieeexplore.ieee.org/lpdocs/epic03/wrapper.htm? arnumber $=1042236$

[234] K. Gilmore, Y. U. Idzerda, M. D. Stiles, Spin-orbit precession damping in transition metal ferromagnets (invited), Journal of Applied Physics 103 (7) (2008) 07D303. doi:10.1063/1.2832348.

URL http://scitation.aip.org/content/aip/journal/jap/103/7/ $10.1063 / 1.2832348$

[235] M. Fähnle, D. Steiauf, J. Seib, The Gilbert equation revisited: anisotropic and nonlocal damping of magnetization dynamics, Journal of Physics D: Applied Physics 41 (16) (2008) 164014. doi: $10.1088 / 0022-3727 / 41 / 16 / 164014$.

URL http://stacks.iop.org/0022-3727/41/i=16/a=164014?key= crossref.f79eefd9c680e748209ecac005be0b57

[236] T. Gilbert, Classics in Magnetics A Phenomenological Theory of Damping in Ferromagnetic Materials, IEEE Transactions on Magnetics 40 (6) (2004) 3443-3449. doi:10.1109/TMAG.2004.836740.

URL http://ieeexplore.ieee.org/lpdocs/epic03/wrapper.htm? arnumber $=1353448$

[237] H. N. Bertram, V. L. Safonov, S. Member, Z. Jin, Thermal Magnetization Noise, Damping Fundamentals, and Mode Analysis : Application to a Thin Film GMR Sensor, IEEE Transactions on Magnetics 38 (5) (2002) 2514-2519.

[238] V. Kamberský, Ferromagnetic resonance in iron whiskers, Can. J. Phys. 48 (1970) 1103.

[239] V. Kamberský, ON FERROMAGNETIC RESONANCE DAMPING IN METALS, Czech. J. Phys., Sect. B 26 (1976) 1366-1383.

[240] V. Kamberský, Spin-orbital Gilbert damping in common magnetic metals, Phys. Rev. B 76 (13) (2007) 134416. doi:10.1103/PhysRevB.76.134416. URL http://link.aps.org/doi/10.1103/PhysRevB.76.134416

[241] K. Gilmore, Y. U. Idzerda, M. D. Stiles, Identification of the Dominant Precession-Damping Mechanism in $\mathrm{Fe}, \mathrm{Co}$, and Ni by First-Principles Calculations, Phys. Rev. Lett. 99 (2) (2007) 027204. doi:10.1103/ PhysRevLett.99.027204. URL http://link.aps.org/doi/10.1103/PhysRevLett.99.027204

[242] K. Gilmore, M. D. Stiles, J. Seib, D. Steiauf, M. Fähnle, Anisotropic damping of the magnetization dynamics in Ni, Co, and Fe, Phys. Rev. B 81 (17) (2010) 174414. doi:10.1103/PhysRevB.81.174414. URL http://link.aps.org/doi/10.1103/PhysRevB.81.174414 
[243] I. Garate, A. MacDonald, Gilbert damping in conducting ferromagnets. I. Kohn-Sham theory and atomic-scale inhomogeneity, Physical Review B 79 (6) (2009) 064403. arXiv:0808.1373, doi:10.1103/PhysRevB.79. 064403.

[244] I. Garate, A. MacDonald, Gilbert damping in conducting ferromagnets. II. Model tests of the torque-correlation formula, Physical Review B Condensed Matter and Materials Physics 79 (6) (2009) 064404. arXiv: arXiv:0808.3923v1, doi:10.1103/PhysRevB.79.064404.

[245] K. Chen, S. Zhang, Spin pumping in the presence of spin-orbit coupling, Physical Review Letters 114 (12) (2015) 126602. arXiv:0706.0370, doi: 10.1103/PhysRevLett.114.126602.

[246] Y. Tserkovnyak, A. Brataas, G. E. W. Bauer, Enhanced Gilbert Damping in Thin Ferromagnetic Films, Phys. Rev. Lett. 88 (11) (2002) 117601. doi:10.1103/PhysRevLett.88.117601. URL http://link.aps.org/doi/10.1103/PhysRevLett.88.117601

[247] Y. Tserkovnyak, A. Brataas, G. E. W. Bauer, Spin pumping and magnetization dynamics in metallic multilayers, Phys. Rev. B 66 (2002) 224403. doi:10.1103/PhysRevB.66.224403. URL http://link.aps.org/doi/10.1103/PhysRevB.66.224403

[248] R. Urban, G. Woltersdorf, B. Heinrich, Gilbert Damping in Single and Multilayer Ultrathin Films: Role of Interfaces in Nonlocal Spin Dynamics, Phys. Rev. Lett. 87 (21) (2001) 217204. doi:10.1103/PhysRevLett. 87. 217204.

URL http://link.aps.org/doi/10.1103/PhysRevLett.87.217204

[249] S. Mizukami, Y. Ando, T. Miyazaki, Effect of spin diffusion on Gilbert damping for a very thin permalloy layer in $\mathrm{Cu} /$ permalloy/Cu/Pt films, Phys. Rev. B 66 (10) (2002) 104413. doi:10.1103/PhysRevB.66.104413. URL http://link.aps.org/doi/10.1103/PhysRevB.66.104413

[250] A. Ghosh, S. Auffret, U. Ebels, W. E. Bailey, Penetration Depth of Transverse Spin Current in Ultrathin Ferromagnets, Phys. Rev. Lett. 109 (12) (2012) 127202. doi:10.1103/PhysRevLett.109.127202. URL http://link.aps.org/doi/10.1103/PhysRevLett.109.127202

[251] B. Heinrich, C. Burrowes, E. Montoya, B. Kardasz, E. Girt, Y.-Y. Song, Y. Sun, M. Wu, Spin Pumping at the Magnetic Insulator (YIG)/Normal Metal (Au) Interfaces, Phys. Rev. Lett. 107 (6) (2011) 066604. doi: 10.1103/PhysRevLett.107.066604.

URL http://link.aps.org/doi/10.1103/PhysRevLett.107.066604

[252] Y. Sun, H. Chang, M. Kabatek, Y.-Y. Song, Z. Wang, M. Jantz, W. Schneider, M. Wu, E. Montoya, B. Kardasz, B. Heinrich, S. G. E. te Velthuis, H. Schultheiss, A. Hoffmann, Damping in Yttrium Iron Garnet 
Nanoscale Films Capped by Platinum, Phys. Rev. Lett. 111 (10) (2013)

106601. doi:10.1103/PhysRevLett.111.106601.

URL http://link.aps.org/doi/10.1103/PhysRevLett.111.106601

[253] S. E. Barnes, S. Maekawa, Generalization of Faraday's Law to Include Nonconservative Spin Forces, Phys. Rev. Lett. 98 (24) (2007) 246601. doi:10.1103/PhysRevLett.98.246601.

URL http://link.aps.org/doi/10.1103/PhysRevLett.98.246601

[254] W. Saslow, Spin pumping of current in non-uniform conducting magnets, Phys. Rev. B 76 (18) (2007) 184434. doi:10.1103/PhysRevB.76.184434. URL http://link.aps.org/doi/10.1103/PhysRevB.76.184434

[255] R. A. Duine, Spin pumping by a field-driven domain wall, Phys. Rev. B 77 (1) (2008) 014409. doi:10.1103/PhysRevB.77.014409. URL http://link.aps.org/doi/10.1103/PhysRevB.77.014409

[256] Y. Tserkovnyak, M. Mecklenburg, Electron transport driven by nonequilibrium magnetic textures, Phys. Rev. B 77 (13) (2008) 134407. doi: 10.1103/PhysRevB.77.134407. URL http://link.aps.org/doi/10.1103/PhysRevB.77.134407

[257] S. Zhang, S. S.-L. Zhang, Generalization of the Landau-Lifshitz-Gilbert Equation for Conducting Ferromagnets, Phys. Rev. Lett. 102 (8) (2009) 086601. doi:10.1103/PhysRevLett.102.086601. URL http://link.aps.org/doi/10.1103/PhysRevLett.102.086601

[258] K. Hals, A. Brataas, Spin-orbit torques and anisotropic magnetization damping in skyrmion crystals, Phys. Rev. B 89 (2014) 064426. doi: 10.1103/PhysRevB.89.064426. URL http://link.aps.org/doi/10.1103/PhysRevB.89.064426

[259] A. Crépieux, C. Lacroix, DzyaloshinskyMoriya interactions induced by symmetry breaking at a surface, Journal of magnetism and magnetic materials 182 (1998) 341-349.

URL http://www.sciencedirect.com/science/article/pii/ S0304885397010445

[260] C. A. Akosa, I. M. Miron, G. Gaudin, A. Manchon, Phenomenology of chiral damping in noncentrosymmetric magnets, Phys. Rev. B 93 (2016) 214429. arXiv:1507.07762, doi:10.1103/PhysRevB.93.214429.

[261] E. Saitoh, M. Ueda, H. Miyajima, G. Tatara, Conversion of spin current into charge current at room temperature: Inverse spin-Hall effect, Applied Physics Letters 88 (18) (2006) 182509. doi:10.1063/1.2199473. URL http://link.aip.org/link/APPLAB/v88/i18/p182509/ $\mathrm{s} 1\{\&\}$ Agg $=$ doi 
[262] S. Emori, U. Bauer, S.-M. Ahn, E. Martinez, G. S. D. Beach, Currentdriven dynamics of chiral ferromagnetic domain walls., Nature materials 12 (7) (2013) 611-6. doi:10.1038/nmat3675.

URL http://www.ncbi.nlm.nih.gov/pubmed/23770726

[263] K.-S. Ryu, L. Thomas, S.-H. Yang, S. Parkin, Chiral spin torque at magnetic domain walls., Nature nanotechnology 8 (7) (2013) 527-33. doi:10.1038/nnano.2013.102.

URL http://www.ncbi.nlm.nih.gov/pubmed/23770808

[264] T. A. Moore, I. M. Miron, G. Gaudin, G. Serret, S. Auffret, B. Rodmacq, A. Schuhl, S. Pizzini, J. Vogel, M. Bonfim, High domain wall velocities induced by current in ultrathin $\mathrm{Pt} / \mathrm{Co} / \mathrm{AlOx}$ wires with perpendicular magnetic anisotropy, Applied Physics Letters 93 (26) (2008) 262504. doi:http://dx.doi.org/10.1063/1.3062855.

URL http://scitation.aip.org/content/aip/journal/apl/93/26/ 10.1063/1.3062855

[265] G. Yu, P. Upadhyaya, X. Li, W. Li, S. K. Kim, Y. Fan, K. L. Wong, Y. Tserkovnyak, P. K. Amiri, K. L. Wang, Room-Temperature Creation and Spin-Orbit Torque Manipulation of Skyrmions in Thin Films with Engineered Asymmetry, Nano Letters 16 (3) (2016) 1981-1988. arXiv: arXiv:1011.1669v3, doi:10.1021/acs.nanolett.5b05257.

[266] J. He, Z. Li, S. Zhang, Current-driven vortex domain wall dynamics by micromagnetic simulations, Phys. Rev. B 73 (18) (2006) 184408. doi: 10.1103/PhysRevB.73.184408. URL http://link.aps.org/doi/10.1103/PhysRevB.73.184408

[267] L. Thomas, M. Hayashi, X. Jiang, R. Moriya, C. Rettner, S. S. P. Parkin, Oscillatory dependence of current-driven magnetic domain wall motion on current pulse length., Nature 443 (7108) (2006) 197-200. doi:10.1038/ nature05093.

URL http://www.ncbi.nlm.nih.gov/pubmed/16971945

[268] M. Eltschka, M. Wötzel, J. Rhensius, S. Krzyk, U. Nowak, M. Kläui, T. Kasama, R. E. Dunin-Borkowski, L. J. Heyderman, H. J. van Driel, R. a. Duine, Nonadiabatic Spin Torque Investigated Using Thermally Activated Magnetic Domain Wall Dynamics, Phys. Rev. Lett. 105 (5) (2010) 056601. doi:10.1103/PhysRevLett.105.056601. URL http://link.aps.org/doi/10.1103/PhysRevLett.105.056601

[269] L. Heyne, J. Rhensius, D. Ilgaz, A. Bisig, U. Rüdiger, M. Kläui, L. Joly, F. Nolting, L. J. Heyderman, J. U. Thiele, F. Kronast, Direct Determination of Large Spin-Torque Nonadiabaticity in Vortex Core Dynamics, Phys. Rev. Lett. 105 (18) (2010) 187203. doi:10.1103/PhysRevLett. 105.187203.

URL http://link.aps.org/doi/10.1103/PhysRevLett.105.187203 
[270] a. Neubauer, C. Pfleiderer, B. Binz, A. Rosch, R. Ritz, P. Niklowitz, P. Böni, Topological Hall Effect in the A Phase of MnSi, Phys. Rev. Lett. 102 (18) (2009) 186602. doi:10.1103/PhysRevLett.102.186602. URL http://link.aps.org/doi/10.1103/PhysRevLett.102.186602

[271] F. Jonietz, S. Mühlbauer, C. Pfleiderer, A. Neubauer, W. Münzer, A. Bauer, T. Adams, R. Georgii, P. Böni, R. a. Duine, K. Everschor, M. Garst, A. Rosch, Spin transfer torques in MnSi at ultralow current densities., Science (New York, N.Y.) 330 (6011) (2010) 1648-51. doi:10.1126/science.1195709. URL http://www.ncbi.nlm.nih.gov/pubmed/21164010

[272] X. Z. Yu, N. Kanazawa, W. Z. Zhang, T. Nagai, T. Hara, K. Kimoto, Y. Matsui, Y. Onose, Y. Tokura, Skyrmion flow near room temperature in an ultralow current density., Nature Communications 3 (2012) 988. doi: $10.1038 /$ ncomms 1990 . URL http://www.ncbi.nlm.nih.gov/pubmed/22871807

[273] A. Fert, V. Cros, J. Sampaio, Skyrmions on the track, Nature nanotechnology 8 (3) (2013) 152-156.

URL http://dx.doi.org/10.1038/nnano.2013.29http:// www. nature.com/nnano/journal/v8/n3/abs/nnano.2013.29. html\{\#\}supplementary-information

[274] J. Iwasaki, M. Mochizuki, N. Nagaosa, Universal current-velocity relation of skyrmion motion in chiral magnets., Nature Communications 4 (2013) 1463. doi: $10.1038 /$ ncomms 2442 .

URL http://www.ncbi.nlm.nih.gov/pubmed/23403564

[275] W. Legrand, D. Maccariello, N. Reyren, K. Garcia, C. Moutafis, C. Moreau-Luchaire, S. Collin, K. Bouzehouane, V. Cros, A. Fert, RoomTemperature Current-Induced Generation and Motion of sub-100 nm Skyrmions, Nano Letters 17 (4) (2017) 2703-2712. arXiv:1702.04616, doi:10.1021/acs.nanolett.7b00649.

[276] S. Zhang, Z. Li, Roles of Nonequilibrium Conduction Electrons on the Magnetization Dynamics of Ferromagnets, Phys. Rev. Lett. 93 (12) (2004) 127204. doi:10.1103/PhysRevLett.93.127204. URL http://link.aps.org/doi/10.1103/PhysRevLett.93.127204

[277] a. Thiaville, Y. Nakatani, J. Miltat, Y. Suzuki, Micromagnetic understanding of current-driven domain wall motion in patterned nanowires, Europhysics Letters (EPL) 69 (6) (2005) 990-996. doi:10.1209/epl/i2004-10452-6.

URL http://stacks.iop.org/0295-5075/69/i=6/a=990?key= crossref.08cfdeef6135f63254ff9d17d22c1198

[278] A. Bisig, C. A. Akosa, J.-H. Moon, J. Rhensius, C. Moutafis, A. von Bieren, J. Heidler, G. Kiliani, M. Kammerer, M. Curcic, M. Weigand, 
T. Tyliszczak, B. Van Waeyenberge, H. Stoll, G. Schütz, K.-J. Lee, A. Manchon, M. Kläui, Enhanced Non-Adiabaticity in Vortex Cores due to the Emergent Hall Effect, Phys. Rev. Lett. 117 (December) (2016) 277203. arXiv:1511.06585, doi:10.1103/PhysRevLett.117.277203. URL http://arxiv.org/abs/1511.06585

[279] G. Tatara, H. Kohno, Theory of Current-Driven Domain Wall Motion: Spin Transfer versus Momentum Transfer, Phys. Rev. Lett. 92 (8) (2004) 086601. doi:10.1103/PhysRevLett.92.086601. URL http://link.aps.org/doi/10.1103/PhysRevLett.92.086601

[280] G. Tatara, H. Kohno, J. Shibata, Y. Lemaho, K.-J. Lee, Spin Torque and Force due to Current for General Spin Textures, Journal of the Physical Society of Japan 76 (5) (2007) 054707. doi:10.1143/JPSJ .76. 054707. URL http://journals.jps.jp/doi/abs/10.1143/JPSJ .76.054707

[281] J. Xiao, A. Zangwill, M. Stiles, Spin-transfer torque for continuously variable magnetization, Phys. Rev. B 73 (5) (2006) 054428. doi:10.1103/ PhysRevB.73.054428. URL http://link.aps.org/doi/10.1103/PhysRevB.73.054428

[282] A. Manchon, K.-J. Lee, Spin Hall effect-driven spin torque in magnetic textures, Applied Physics Letters 99 (2) (2011) 022504. doi:10.1063/1.3609236.

URL http://scitation.aip.org/content/aip/journal/apl/99/2/ $10.1063 / 1.3609236$

[283] C. A. Akosa, W.-S. Kim, A. Bisig, M. Kläui, K.-J. Lee, A. Manchon, Role of spin diffusion in current-induced domain wall motion for disordered ferromagnets, Phys. Rev. B 91 (2015) 094411. doi:10.1103/PhysRevB. 91.094411. URL http://link.aps.org/doi/10.1103/PhysRevB.91.094411

[284] C. A. Akosa, P. B. Ndiaye, A. Manchon, Intrinsic non-adiabatic topological torque in magnetic skyrmions and vortices, Phys. Rev. B 95 (2017) 054434. arXiv:1609.09606, doi:10.1103/PhysRevB.95.054434. URL http://arxiv.org/abs/1609.09606

[285] K. Everschor, M. Garst, B. Binz, F. Jonietz, S. Mühlbauer, C. Pfleiderer, A. Rosch, Rotating skyrmion lattices by spin torques and field or temperature gradients, Phys. Rev. B 86 (5) (2012) 054432. doi: 10.1103/PhysRevB.86.054432. URL http://link.aps.org/doi/10.1103/PhysRevB.86.054432

[286] M. Berry, Quantal phase factors accompanying adiabatic changes, Proceedings of the Royal Society of London. Series A, Mathematical and Physical Sciences 392 (1802) (1984) 45-57. 
[287] J. Ye, Y. B. Kim, A. J. Millis, B. I. Shraiman, P. Majumdar, Z. Te, Berry Phase Theory of the Anomalous Hall Effect : Application to Colossal Magnetoresistance Manganites, Phys. Rev. Lett. 83 (1999) 3737.

[288] Y. Taguchi, Y. Oohara, H. Yoshizawa, N. Nagaosa, Y. Tokura, Spin chirality, Berry phase, and anomalous Hall effect in a frustrated ferromagnet., Science (New York, N.Y.) 291 (5513) (2001) 2573-6. doi: 10.1126/science. 1058161. URL http://www.ncbi.nlm.nih.gov/pubmed/11283363

[289] G. E. Volovik, Linear momentum in ferromagnets, Journal of Physics C: Solid State Physics 20 (7) (1987) L83. URL http://stacks.iop.org/0022-3719/20/i=7/a=003

[290] P. Bruno, V. Dugaev, M. Taillefumier, Topological Hall Effect and Berry Phase in Magnetic Nanostructures, Phys. Rev. Lett. 93 (9) (2004) 096806. doi:10.1103/PhysRevLett.93.096806. URL http://link.aps.org/doi/10.1103/PhysRevLett.93.096806

[291] Y. Tserkovnyak, A. Brataas, G. E. W. Bauer, Theory of currentdriven magnetization dynamics in inhomogeneous ferromagnets, Journal of Magnetism and Magnetic Materials 320 (7) (2008) 1282-1292. doi:10.1016/j.jmmm.2007.12.012.

URL http://linkinghub.elsevier.com/retrieve/pii/ S030488530701013X

[292] A. Stern, Berrys phase, motive forces, and mesoscopic conductivity, Physical Review Letters 68 (7) (1992) 1022-1025. arXiv:arXiv:1011.1669v3, doi:10.1103/PhysRevLett.68.1022.

[293] K. Tanabe, D. Chiba, J. Ohe, S. Kasai, H. Kohno, S. E. Barnes, S. Maekawa, K. Kobayashi, T. Ono, Spin-motive force due to a gyrating magnetic vortex, Nature Communications 3 (May) (2012) 845. doi: $10.1038 /$ ncomms 1824 URL http://dx.doi.org/10.1038/ncomms1824

[294] Y. Shimada, J. I. Ohe, Spin motive force driven by skyrmion dynamics in magnetic nanodisks, Physical Review B 91 (17) (2015) 174437. doi: 10.1103/PhysRevB.91.174437.

[295] J. Zang, M. Mostovoy, J. Han, N. Nagaosa, Dynamics of Skyrmion Crystals in Metallic Thin Films, Phys. Rev. Lett. 107 (13) (2011) 136804. doi:10.1103/PhysRevLett.107.136804. URL http://link.aps.org/doi/10.1103/PhysRevLett.107.136804

[296] P. B. N'diaye, C. A. Akosa, A. Manchon, Topological Hall and Spin Hall Effects in Disordered Skyrmionic Textures, Phys. Rev. B 95 (2017) 064426. arXiv:1609.05480, doi:10.1103/PhysRevB.95.064426. URL http://arxiv.org/abs/1609.05480 
[297] A. A. Thiele, Steady-State Motin of Magnetic Domains, Phys. Rev. Lett. 30 (6) (1973) 230.

[298] T. Schulz, R. Ritz, A. Bauer, M. Halder, M. Wagner, C. Franz, C. Pfleiderer, K. Everschor, M. Garst, A. Rosch, Emergent electrodynamics of skyrmions in a chiral magnet, Nature Physics 8 (4) (2012) 301-304. doi:10.1038/nphys2231.

URL http://www. nature.com/doifinder/10.1038/nphys2231

[299] T. S. MacHado, T. G. Rappoport, L. C. Sampaio, Static and dynamic properties of vortices in anisotropic magnetic disks, Applied Physics Letters 93 (11) (2008) 112507. doi:10.1063/1.2985901.

[300] F. Garcia, H. Westfahl, J. Schoenmaker, E. J. Carvalho, A. D. Santos, M. Pojar, A. C. Seabra, R. Belkhou, A. Bendounan, E. R. P. Novais, A. P. Guimarães, Tailoring magnetic vortices in nanostructures, Applied Physics Letters 97 (2) (2010) 022501. doi:10.1063/1.3462305.

[301] E. Jué, A. Thiaville, S. Pizzini, J. Miltat, J. Sampaio, L. D. BudaPrejbeanu, S. Rohart, J. Vogel, M. Bonfim, O. Boulle, S. Auffret, I. M. Miron, G. Gaudin, Domain wall dynamics in ultrathin $\mathrm{Pt} / \mathrm{Co} / \mathrm{AlOx}$ microstrips under large combined magnetic fields, Physical Review B 93 (1) (2016) 014403. doi:10.1103/PhysRevB.93.014403.

[302] T. Jungwirth, X. Marti, P. Wadley, J. Wunderlich, Antiferromagnetic spintronics, Nature Nanotechnology 11 (3) (2016) 231-241. arXiv:arXiv: 1509.05296v1, doi:10.1038/nnano.2016.18. URL http://www.nature.com/doifinder/10.1038/nnano.2016.18

[303] Y. T. V. Baltz, A. Manchon, M. Tsoi, T. Moriyama, T. Ono, Antiferromagnetism: the next flagship magnetic order for spintronics ?, ArXiv1606.04284.

[304] A. Núñez, R. Duine, P. Haney, A. MacDonald, Theory of spin torques and giant magnetoresistance in antiferromagnetic metals, Phys. Rev. B 73 (April) (2006) 214426. doi:10.1103/PhysRevB.73.214426.

URL http://journals.aps.org/prb/abstract/10.1103/PhysRevB . 73.214426

[305] J. Železný, H. Gao, K. Výborný, J. Zemen, Relativistic Néel-Order Fields Induced by Electrical Current in Antiferromagnets, Physical Review Letters 113 (15) (2014) 157201. doi:10.1103/PhysRevLett.113.157201. URL http://link.aps.org/doi/10.1103/PhysRevLett.113. 157201http://journals.aps.org/prl/abstract/10.1103/ PhysRevLett.113.157201

[306] P. Wadley, B. Howells, J. Elezny, C. Andrews, V. Hills, R. P. Campion, V. Novak, K. Olejnik, F. Maccherozzi, S. S. Dhesi, S. Y. Martin, T. Wagner, J. Wunderlich, F. Freimuth, Y. Mokrousov, J. Kune, 
J. S. Chauhan, M. J. Grzybowski, A. W. Rushforth, K. W. Edmonds, B. L. Gallagher, T. Jungwirth, Electrical switching of an antiferromagnet, Science 351 (6273) (2016) 587-590. arXiv:1503.03765, doi:10.1126/science.aab1031.

URL http://arxiv.org/abs/1503.03765\{\%\}5Cnhttp://www . sciencemag.org/cgi/doi/10.1126/science.aab1031

[307] J. Železný, H. Gao, A. Manchon, F. Freimuth, Y. Mokrousov, J. Zemen, J. Mašek, J. Sinova, T. Jungwirth, Spin-orbit torques in locally and globally non-centrosymmetric crystals: Antiferromagnets and ferromagnets, Phys. Rev. B 95 (2017) 014403. arXiv:1604.07590, doi: 10.1103/PhysRevB.95.014403. URL http://arxiv.org/abs/1604.07590

[308] A. Manchon, Spin diffusion and torques in disordered antiferromagnets, J. Phys.: Condens. Matter 29 (2017) 104002. doi:10.1088/1361-648X/ aa521d.

[309] X. Zhang, Y. Zhou, M. Ezawa, Antiferromagnetic Skyrmion: Stability, Creation and Manipulation, Sci. Rep. (2015) 6:24795arXiv:1504.01198, doi: $10.1038 /$ srep24795. URL http://arxiv.org/abs/1504.01198

[310] J. Barker, O. A. Tretiakov, Static and Dynamical Properties of Antiferromagnetic Skyrmions in the Presence of Applied Current and Temperature, Physical Review Letters 116 (14) (2016) 147203. arXiv:1505.06156, doi:10.1103/PhysRevLett.116.147203.

[311] M. Z. Hasan, C. Kane, Colloquium: Topological insulators, Rev. Mod. Phys. 82 (4) (2010) 3045-3067. doi:10.1103/RevModPhys.82.3045. URL http://link.aps.org/doi/10.1103/RevModPhys.82.3045

[312] X.-L. Qi, S.-C. Zhang, Topological insulators and superconductors, Rev. Mod. Phys. 83 (4) (2011) 1057-1110. doi:10.1103/RevModPhys.83.1057. URL http://link.aps.org/doi/10.1103/RevModPhys.83.1057

[313] T. Wehling, A. Black-Schaffer, A. Balatsky, Dirac materials, Advances in Physics 63 (1) (2014) 1-76. doi:10.1080/00018732.2014.927109. URL http://www.tandfonline.com/doi/abs/10.1080/00018732. 2014.927109

[314] L. Fu, Topological Crystalline Insulators, Phys. Rev. Lett. 106 (10) (2011) 106802. doi:10.1103/PhysRevLett.106.106802.

URL http://link.aps.org/doi/10.1103/PhysRevLett.106.106802

[315] J. Li, R.-L. Chu, J. K. Jain, S.-Q. Shen, Topological Anderson Insulator, Physical Review Letters 102 (13) (2009) 136806. arXiv:0811.3045, doi: 10.1103/PhysRevLett.102.136806. URL http://link.aps.org/doi/10.1103/PhysRevLett.102.136806 
[316] C. W. Groth, M. Wimmer, A. R. Akhmerov, J. Tworzydło, C. W. J. Beenakker, Theory of the Topological Anderson Insulator, Physical Review Letters 103 (19) (2009) 1-4. arXiv:0908.0881, doi:10.1103/ PhysRevLett.103.196805.

[317] M. Dzero, J. Xia, V. Galitski, P. Coleman, Topological Kondo Insulators, Annual Review of Condensed Matter Physics 7 (March) (2016) 249. arXiv:1506.05635, doi:10.1146/)).

URL http://arxiv.org/abs/1406.3533\{\%\}5Cnhttp://arxiv.org/ abs/1506.05635

[318] X. Wan, A. M. Turner, A. Vishwanath, S. Y. Savrasov, Topological semimetal and Fermi-arc surface states in the electronic structure of pyrochlore iridates, Phys. Rev. B 83 (20) (2011) 205101. doi:10.1103/ PhysRevB.83.205101.

URL http://link.aps.org/doi/10.1103/PhysRevB.83.205101

[319] A. A. Soluyanov, D. Gresch, Z. Wang, Q. Wu, M. Troyer, X. Dai, B. A. Bernevig, Type-II Weyl semimetals, Nature 527 (7579) (2015) 495-498. arXiv:1507.01603, doi:10.1038/nature15768. URL http://dx.doi.org/10.1038/nature15768

[320] S. M. Young, S. Zaheer, J. C. Y. Teo, C. L. Kane, E. J. Mele, A. M. Rappe, Dirac semimetal in three dimensions, Physical Review Letters 108 (14) (2012) 140405. arXiv:arXiv:1111.6483v2, doi:10.1103/PhysRevLett. 108.140405. 